Aus der Abteilung Gastroenterologie und Endokrinologie

(Prof. Dr. med. Dr. h.c. G. Ramadori)

Im Zentrum Innere Medizin

Der Medizinischen Fakultät der Universität Göttingen

\title{
Diagnostik und Therapie des Polyzystischen Ovarsyndroms im Rahmen der klinischen Routineversorgung
}

\author{
INAUGURAL-DISSERTATION \\ zur Erlangung des Doktorgrades \\ der Medizinischen Fakultät \\ der Georg-August-Universität zu Göttingen
}

vorgelegt von

Björn Wiesemann

aus

Bad Wildungen

Göttingen 2012 
Dekan:

1. Berichterstatterin:

2. Berichterstatter:

Tag der mündlichen Prüfung:
Prof. Dr. med. C. Frömmel

Prof. Dr. med. H. Siggelkow

Prof. Dr. Dr. med. B. Hinney

10.10.2012 


\section{Inhaltsverzeichnis}

Abkürzungsverzeichnis

\section{Einleitung}

1.1 Das Polyzystische Ovarsyndrom (PCOS): Einführung und Epidemiologie

1.2 Historische Entwicklung und aktuelle Definition 1

1.3 Pathogenese und Klinik ___ 2

1.3.1 Hyperandrogenismus __ 2

1.3.2 Polyzystische Ovarien __ 3

1.3.3 Gestörte neuroendokrine Funktion - LH/FSH-Quotient ___ 3

1.3.4 Insulinresistenz __ 4

1.3.5 Lebensstil und Vitamin-D-Mangel___ 6

1.3.6 Genetik _ 6

1.4 Diagnostik__ 7

1.4.1 Anamnese___ 7

1.4.2 Hyperandrogenismus __ 7

1.4.3 Polyzystische Ovarien __ 8

1.4.4 Hormondiagnostik___ 8

1.4.5 Insulinresistenz _ 9

1.5 Therapie _ـ 11

1.5.1 Überblick__ 11

1.5.2 Hyperandrogenismus ___ 11

1.5.3 Zyklusstörungen und Ovulationsinduktion bei Infertilität___ 12

1.5.4 Insulinresistenz _ 12

1.6 Begleit- und Folgeerkrankungen des PCOS __ 14

1.6.1 Diabetes mellitus, Metabolisches Syndrom und kardiovaskuläres Risiko _ 14

1.6.2 Maligne gynäkologische Tumoren _ـ 15

1.6.3 Weitere Erkrankungen und Risiken _ 16

1.7 Fragestellung _ 17

2. Patienten, Material und Methoden__ 18

2.1 Studiendesign 18

2.2 Patientenauswahl und Gruppeneinteilung __ 18

2.3 Methoden 20

2.3.1 Datengewinnung__ 20

2.3.2 Diagnose PCOS __ 21

2.3.3 Diagnostik der Insulinresistenz___ 21

2.3.3.1 Oraler Glukosetoleranztest (OGTT) _ 21

2.3.3.2 Homeostasis Model Assessment Test (HOMA) _ 22 


\subsubsection{Quantitative insulin sensitivity check index (QUICKI)}

23

2.3.4 Klinische Hyperandrogenämie _ 23

2.3.5 Laboruntersuchungen__ 23

2.3.6 Nebendiagnosen__ 25

2.3.7 Statistik_ 25

3. Ergebnisse__ 27

3.1 Hinweise zur Patientencharakterisierung _ 27

3.2 Charakterisierung der Gruppen 1-5 27

3.2.1 Klinische Basisparameter__ 27

3.2.1.1 Alter_ 28

3.2.1.2 BMI_ 28

3.3 Patientinnencharakterisierung nach Gruppenzusammenfassung ___ 31

3.3.1 Gesamtkollektiv nach endgültiger Gruppenverteilung __ 31

3.3.2 Klinische Basisparameter___ 31

3.3.2.1 Alter_ 32

3.3.2.2 BMI 33

3.3.3 Klinische PCOS-Parameter __ 33

3.3.3.1 Akne 33

3.3.3.2 Hirsutismus _ 33

3.3.3.3 Zyklusstörungen _ 34

3.3.4 Besondere Nebendiagnosen und Medikation__ 35

3.3.4.1 Hashimoto-Thyreoiditis (AIT)_ 35

3.3.4.2 Vormedikation mit Ovulationshemmern _ 35

3.3.5 Ergebnisse des OGTT_ 35

3.3.5.1 Glukose, Insulin und C-Peptid___ 36

3.3.5.2 AUC Glukose, AUC Insulin, AUC C-Peptid___ 37

3.3.5.3 HOMA-IR und QUICKI _ 38

3.3.6 Einfluss von Ovulationshemmern__ 38

3.3.6.1 Ovulationshemmer bei PCOS-Patientinnen _ 38

3.3.6.2 Ovulationshemmer bei Patientinnen ohne PCOS __ 39

3.3.7 LH/FSH, SHBG und $\mathrm{FAI} \_40$

3.3.7.1 LH/FSH_ 40

3.3.7.2 SHBG 41

3.3.7.3 FAl_ 43

3.4 Therapie der Insulinresistenz bei PCOS-Patientinnen mit Metformin __ 44

3.4.1 Klinische Basisparameter _ 45

3.4.1.1 Alter__ 45

3.4.1.2 Gewicht und BMI___ 45 
3.4.2 Klinische PCOS-Parameter 47

3.4.2.1 Akne 47

3.4.2.2 Hirsutismus _ 47

3.4.2.3 Zyklusstörungen _ 47

3.4.3 Ergebnisse des ersten und zweiten OGTT im Vergleich __ 48

3.4.3.1 Glukose, Insulin und C-Peptid___ 48

3.4.3.2 AUC Glukose, AUC Insulin, AUC C-Peptid__ 50

3.4.3.3 HOMA-IR und QUICKI 50

3.4.4 Einfluss von Ovulationshemmern _ 51

3.4.5 FAl und SHBG _ 52

3.4.5.1 $\mathrm{FAl} \quad 53$

3.4.5.2 SHBG 54

4. Diskussion_ 55

4.1 Einfluss von Ovulationshemmern _ 55

4.2 Vergleich der Gruppen PCOS und Ausschluss PCOS__ 56

4.2.1 Alter und BMI 56

4.2.2 Akne, Hirsutismus und Zyklusstörungen _ 57

4.2.3 Hashimoto-Thyreoiditis (AIT) _ 59

4.2.4 LH/FSH_ 60

4.2.5 SHBG _ 62

4.2.6 FAl_ 64

4.2.7 OGTT bei PCOS-Patientinnen und bei Patientinnen ohne PCOS __ 66

4.3 Therapie mit Metformin bei 30 PCOS-Patientinnen ___ 69

4.3.1 $\mathrm{BMI} 69$

4.3.2 Akne, Hirsutismus und Zyklusstörungen _ 71

4.3.3 FAl und SHBG _ 72

4.3.4 OGTT und Insulinresistenz __ 73

4.4 Kritik und Ausblick _ 77

5. Zusammenfassung _ 79

6. Anhang_ 82

6.1 Abbildungsverzeichnis __ 82

6.2 Tabellenverzeichnis _ 83

6.3 Patienteninformation Metformin _ 84

6.4 Ferriman-Gallwey-Score __ 85

7. Literaturverzeichnis 86 


\section{Abkürzungsverzeichnis}

\begin{tabular}{|c|c|}
\hline AlT & Autoimmunthyreoiditis (= Hashimoto-Thyreoiditis) \\
\hline AUC & Area under the curve (Fläche unter der Kurve) \\
\hline BMI & Body mass index \\
\hline CHOD-PAP & $\begin{array}{l}\text { CHOD-PAP-Methode = Enzymatischer Farbtest zur Cholesterin- } \\
\text { bestimmung }\end{array}$ \\
\hline CLIA & Chemilumineszenz Immuno Assay \\
\hline CRP & C-reaktives Protein \\
\hline DHEA-S & Dehydroepiandrosteron-Sulfat \\
\hline ECL & Elektrochemilumineszenz \\
\hline ECLIA & Elektro Chemilumineszenz Immuno Assay \\
\hline FAl & Freier Androgenindex \\
\hline FSH & Follikel-stimulierendes Hormon \\
\hline GNRH & Gonadotropin-Releasing-Hormon \\
\hline HDL & High-Density-Lipoprotein \\
\hline HOMA-IR & Homeostasis Model Assessment Test (für Insulinresistenz) \\
\hline IGT & Gestörte Glukosetoleranz \\
\hline IVF & In-vitro-Fertilisation \\
\hline LDL & Low-density Lipoprotein \\
\hline LH & Luteinisierendes Hormon \\
\hline NGT & Normale Glukosetoleranz \\
\hline n.s. & Nicht signifikant \\
\hline OGTT & Oraler Glukosetoleranztest \\
\hline PCOS & Polyzystisches Ovarsyndrom \\
\hline РHOT & Photometrie \\
\hline QUICKI & Quantitative insulin sensitivity check index \\
\hline RIA & Radioimmunoassay \\
\hline SD & Standardabweichung \\
\hline SHBG & Sexualhormon-bindendes Globulin \\
\hline TG & Thyreoglobulin \\
\hline TPO & Thyreoperoxidase=lodid-Peroxidase \\
\hline TSH & Thyroidea-stimulierendes Hormon \\
\hline UAW & Unerwünschte Arzneimittelwirkung \\
\hline
\end{tabular}




\section{Einleitung}

\subsection{Das Polyzystische Ovarsyndrom (PCOS): Einführung und Epidemiologie}

Das Polyzystische Ovarsyndrom - nachfolgend stets nur noch als PCOS benannt ist eine der häufigsten endokrinologischen Erkrankungen von Frauen im gebärfähigen Alter (Azziz et al. 2004). Je nach betrachteter geographischer Region sind etwa 6 bis $10 \%$ aller Frauen betroffen (Goodarzi et al. 2011). Dabei findet sich eine höhere Prävalenz bei übergewichtigen und adipösen Frauen (Alvarez-Blasco et al. 2006). Metabolische Störungen, insbesondere die Insulinresistenz mit erhöhtem Risiko für die Entwicklung eines Diabetes mellitus und kardiovaskulärer Erkrankungen, sind bei PCOS-Patientinnen von Bedeutung (Goodarzi et al. 2011).

Das PCOS ist somit durch komplexe endokrine Störungen mit einer Vielzahl an klinischen Manifestationen und Spätfolgen gekennzeichnet. Anhand der historischen Entwicklung einer klinischen Definition wird im Folgenden das PCOS näher erläutert.

\subsection{Historische Entwicklung und aktuelle Definition}

Bereits im 18. Jahrhundert erkannten Ärzte den Zusammenhang verschiedener Symptome des PCOS (Tan et al. 2008). Zu Beginn des 20. Jahrhunderts entdeckten französische Internisten den Zusammenhang von Diabetes mellitus bei behaarten Frauen im Sinne eines Hyperandrogenismus. Sie postulierten den „diabète des femmes à barbes" (Achard und Thiers 1921; Tan et al. 2008). Einen Meilenstein in der Entwicklung einer Definition des PCOS gelang den amerikanischen Gynäkologen Stein und Leventhal im Jahre 1935, die erstmals die Trias aus Zyklusstörungen, Hyperandrogenismus und den fortan namensgebenden Polyzystischen Ovarien beschrieben (Stein und Leventhal 1935). Das PCOS wurde künftig auch als SteinLeventhal-Syndrom bezeichnet.

Eine exakte Definition erfuhr das PCOS erst in den letzten beiden Dekaden.

Im Jahre 1990 legte die Konsensuskonferenz des NIH (National Institutes of Health) eine chronische Anovulation bei Oligo-/Amenorrhoe sowie eine klinische und/oder laborchemische Hyperandrogenämie als diagnostische Kriterien des PCOS fest, 
wobei Differenzialdiagnosen bezüglich Erkrankungen des Ovars, der Hypophyse und der Nebenniere auszuschließen sind (Carmina 2004; Zawadski und Dunaif 1992). Polyzystische Ovarien stellten kein Diagnosekriterium mehr dar. Diese wurden wieder in die Definition des PCOS miteinbezogen, als 2003 die European Society of Human Reproduction and Embryology (ESHRE) und die American Society of Reproductive Medicine (ASRM) gemeinsam als The Rotterdam ESHRE/ASRMsponsored PCOS consensus workshop group die sogenannten Rotterdam-Kriterien festlegten (2004a; 2004b). Bei Präsenz von zwei der drei Kriterien (Anovulation, Hyperandrogenismus und Polyzystische Ovarien) nach Ausschluss von Differenzialdiagnosen liegt hierbei ein PCOS vor.

Die aktuelle Leitlinie der Androgen Excess Society (AES) zur Definition des PCOS ist eine Weiterentwicklung der $\mathrm{NIH}$ - und Rotterdamkriterien. Sie betont jedoch den Aspekt des Hyperandrogenismus als essenzielles Element in der Pathogenese des PCOS (Azziz et al. 2006). Eine aktuelle Übersichtsarbeit kommt zum gleichen Ergebnis, wenngleich auch eine Minderheit die Meinung vertritt, PCOS existiere auch ohne Hyperandrogenismus (Azziz et al. 2009).

Tabelle 1 fasst die Diagnosekriterien nochmals zusammen.

\begin{tabular}{|c|c|c|}
\hline $\begin{array}{l}\text { NIH-Konsensus, } 1990 \\
\text { (Carmina 2004) }\end{array}$ & Rotterdam-Kriterien, 2003 & $\begin{array}{c}\text { Leitlinie AES, } 2006 \\
\text { (Azziz et al. 2006) }\end{array}$ \\
\hline $\begin{array}{ll}\text { - } & \text { Chron. Anovulation } \\
& \text { (Oligo-/Amenorrhoe) } \\
\text { - } & \text { Klinische und/oder } \\
& \text { laborchemische } \\
& \text { Hyperandrogenämie } \\
\rightarrow & \text { beide Kriterien }\end{array}$ & $\begin{array}{ll}\text { - } & \text { Chron. Anovulation } \\
\text { (Oligo-/Amenorrhoe) }\end{array}$ & $\begin{array}{l}\text { - } \frac{\text { Immer klinische u./o. }}{\text { laborchemische }} \\
\text { Hyperandrogenämie } \\
\rightarrow \text { entweder mit } \\
\text { - Chron. Anovulation } \\
\text { (Oligo-/Amenorrhoe) } \\
\Rightarrow \text { und/oder } \\
\text { - Polyzystische Ovarien }\end{array}$ \\
\hline
\end{tabular}

Tabelle 1: Vergleich der Diagnose-Kriterien des PCOS.

\subsection{Pathogenese und Klinik}

\subsubsection{Hyperandrogenismus}

Der Begriff Hyperandrogenismus meint den Zustand erhöhter Androgene im Blut mit möglichen klinischen Symptomen. Der Hyperandrogenismus spielt eine bedeutende 
Rolle in der Pathogenese des PCOS, wenngleich nicht bei allen Patientinnen mit klinischen Symptomen einer Hyperandrogenämie ein PCOS vorliegt (Azziz et al. 2009). Laborchemisch finden sich in ca. $75 \%$ aller PCOS-Patientinnen erhöhte Androgene im Blut (Huang et al. 2010). Gesamttestosteron und DHEAS sind dabei erhöht. In vielen Fällen ist zudem der Anteil des freien Testosterons am Gesamttestosteron (freier Androgenindex, FAI) erhöht. Klinisch manifestiert sich eine Hyperandrogenämie in Form von Hirsutismus, Akne und Alopezie vom androgenen Typ. Allerdings liegt der Anteil an Frauen mit PCOS, die unter Hirsutismus leiden, je nach betrachteter Region und verwendeter Definition des PCOS nur bei 65 bis $75 \%$. Das Auftreten von Akne und androgenetischer Alopezie ist noch seltener (Azziz et al. 2009; Lowenstein 2006).

\subsubsection{Polyzystische Ovarien}

Etwa 75 bis 90 \% der PCOS-Patientinnen haben auch Polyzystische Ovarien (Azziz et al. 2009). Zur Definition siehe Abschnitt 1.4.3, S. 8.

Sowohl eine Hyperandrogenämie als auch eine Hyperinsulinämie nehmen Einfluss auf die Morphologie des Ovars. Zunächst stimulieren Androgene erheblich das Follikelwachstum. Im Verlauf wird die Follikelreifung jedoch durch intrafollikuläre Androgene und Hyperinsulinämie gestört und endet schließlich im Follikelarrest (Goodarzi et al. 2011; Jonard et al. 2003). Pathophysiologisch wird derzeit eine Vielzahl an molekularen Mechanismen diskutiert, wobei die Hyperinsulinämie eine besondere Bedeutung hat. Insulin erhöht die Produktion von Androgenen einerseits durch LH- und IGF-1-Stimulation, andererseits durch verminderte Aktivität des SHBG mit resultierendem Anstieg des freien Testosterons (Goodarzi et al. 2011).

Die so entstehende typische perlschnurartige PCO-Morphologie führt klinisch zu Zyklusstörungen in Form von Oligo- und Amenorrhoe und meist auch zu anovulatorischer Infertilität (Schuring et al. 2008).

\subsubsection{Gestörte neuroendokrine Funktion - LH/FSH-Quotient}

Beim PCOS ist der Regelkreis der hypothalamisch-hypophysären Achse bis hin zum Ovar gestört. Bedingt durch eine erhöhte GnRH-Pulsfrequenz lässt sich beim PCOS 
eine erhöhte Frequenz und Amplitude der LH-Ausschüttung nachweisen, was schließlich zu unphysiologisch hohen LH-Werten im Plasma führt (Blank et al. 2006). Pulsfrequenz und Plasmaspiegel von FSH bleiben dagegen nahezu physiologisch, so dass insgesamt ein um den Faktor zwei bis drei erhöhter LH/FSH-Quotient resultiert (Goodarzi et al. 2011). Bei Ausschluss von Frauen mit kürzlich stattgefundenem Eisprung haben $94 \%$ aller PCOS-Patientinnen einen erhöhten LH/FSH-Quotienten (Taylor et al. 1997). Der BMI ist negativ mit den Plasmawerten und der Pulsamplitude von LH korreliert, wodurch erhöhte LH/FSH-Quotienten eher bei normalgewichtigen PCOS-Patientinnen beobachtet werden (Págan et al. 2006).

Pathophysiologisch existiert ein sich selbst unterhaltender Kreislauf. Die unter $\mathrm{LH}$ verstärkte Produktion von Androgenen und Progesteron im Ovar führt nicht zu verminderter $\mathrm{GnRH}$-Freisetzung im Hypothalamus. Als Ursache der gestörten negativen Rückkopplung mit verminderter Sensitivtät des Progesterons im Hypothalamus wird die unter anderem durch LH verursachte Hyperandrogenämie selbst vermutet (Blank et al. 2006). Bei kombinierter Gabe des Androgen-RezeptorAntagonisten Flutamid zusammen mit Östradiol und Progesteron wird eine verminderte LH-Pulsfrequenz beobachtet (Eagleson et al. 2000). Die Dauer einer Hyperandrogenämie und genetische Faktoren scheinen dabei einen Einfluss auf die Ausprägung der gestörten Rückkopplung zu haben (Chhabra et al. 2005).

\subsubsection{Insulinresistenz}

Unter einer Insulinresistenz versteht man den meist im Kontext von Adipositas gestörten Blutzuckerstoffwechsel, in welchem die physiologische InsulinAusschüttung zu keiner adäquaten Senkung des Blutzuckers führt. In der Folge werden höhere Mengen an Insulin benötigt. Trotz zunehmenden Verständnisses der pathophysiologischen Zusammenhänge existiert derzeit keine allgemeingültige Definition der Insulinresistenz, was auch zu Kontroversen bezüglich diagnostischer Standards führt (siehe Abschnitt 1.4.5, S. 9)(Traub 2011).

Insulinresistenz mit nachfolgender erhöhter Insulinsekretion des Pankreas und daraus resultierender Hyperinsulinämie nehmen in der Pathophysiologie des PCOS eine zunehmend kausale Rolle ein (Azziz et al. 2009). Unter Berücksichtigung des Alters, der Ethnie und der Messmethode liegt bei etwa 60 bis $80 \%$ der PCOS- 
Patientinnen eine Insulinresistenz vor (Carmina und Lobo 2004; DeUgarte et al. 2005; Ovalle und Azziz 2002). PCOS-Patientinnen mit Diabetes mellitus Typ 2 in der Familienanamnese haben häufiger eine Insulinresistenz und gestörte Glukosetoleranz als PCOS-Patientinnen ohne familiäre Disposition hierfür (Ehrmann et al. 2005). Im Vergleich zu gesunden Patientinnen finden sich bei PCOSPatientinnen höhere Insulinwerte und eine höhere Inzidenz der Insulinresistenz (Carmina et al. 2007). Dabei erklären Übergewicht, Adipositas und BMI nicht den Unterschied in beiden Gruppen, da auch schlanke PCOS-Patientinnen eine im Vergleich höhere Insulinresistenz haben (Dunaif et al. 1989).

Die der Insulinresistenz beim PCOS zu Grunde liegenden molekularen Mechanismen sind noch nicht umfassend geklärt. Vermutet werden unter anderem $\beta$-Zell-Defekte und Störungen der Insulinsekretion sowie der Lipolyse in Adipozyten (Goodarzi et al. 2011; Tan et al. 2008).

Wie in Abschnitt 1.3.2 beschrieben sind Hyperinsulinämie und Hyperandrogenämie positiv miteinander korreliert. Das in diesem Kontext durch Insulin erniedrigte SHBG findet auch Verwendung als zuverlässiger Marker der Insulinresistenz und als Prädiktor von Diabetes mellitus Typ 2 (Schuring et al. 2008).

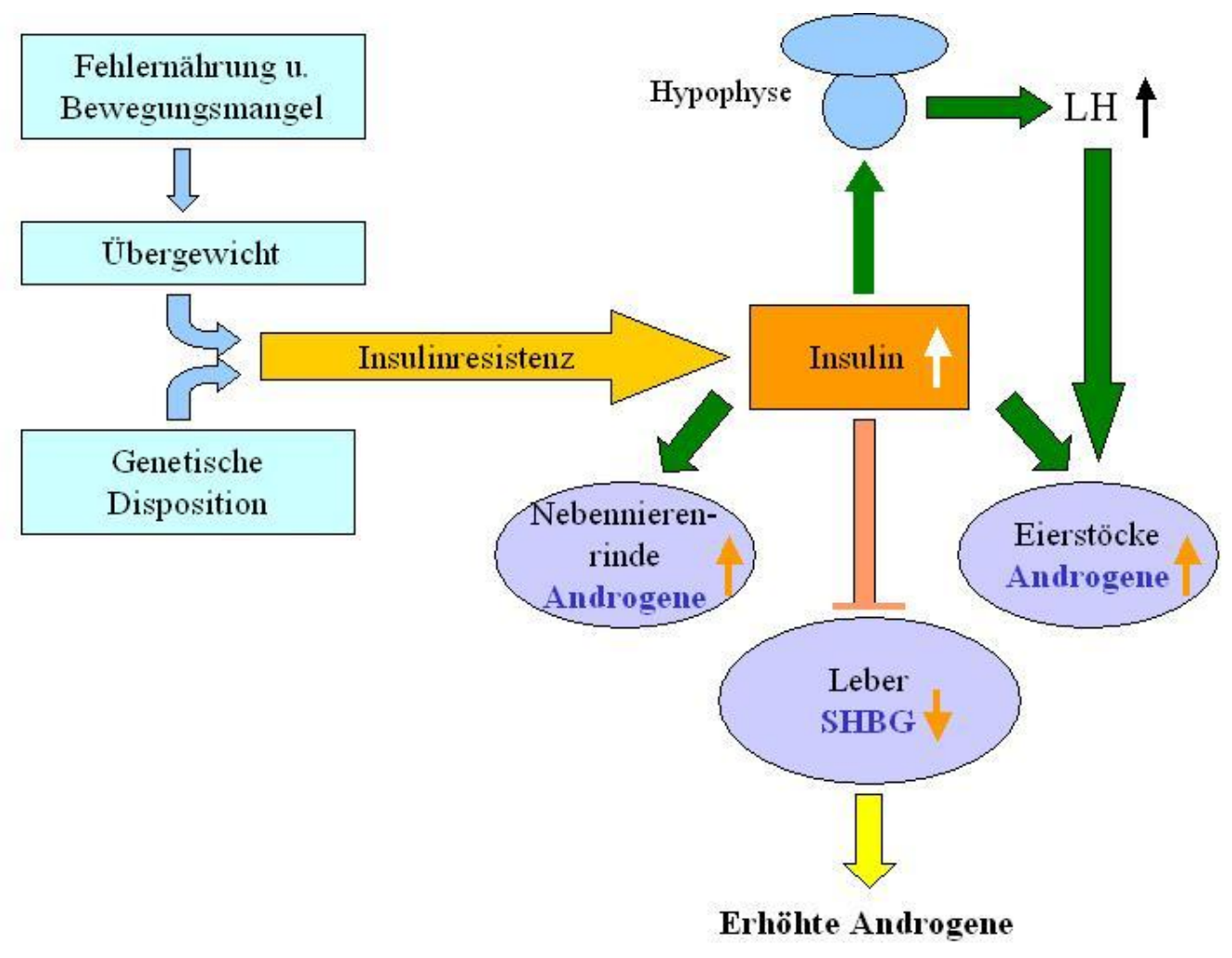

Abbildung 1: Auswirkungen der Insulinresistenz beim PCOS. (Abbildung mit freundlicher Genehmigung von Prof. Dr. Dr. med. B. Hinney, Universitätsfrauenklinik Göttingen) 


\subsubsection{Lebensstil und Vitamin-D-Mangel}

Der Lebensstil und auch Umweltfaktoren beeinflussen die Klinik des PCOS.

Im Zuge allgemeiner gesellschaftlicher Entwicklungen hat der Grad an Übergewicht und Adipositas sowohl in der gesunden Bevölkerung ohne PCOS als auch bei PCOS-Patientinnen zugenommen, so dass keine signifikant höhere Prävalenz für Adipositas bei PCOS-Patientinnen existiert (Yildiz et al. 2008). Das regional unterschiedlich gehäufte Auftreten von Adipositas bei PCOS-Patientinnen beruht mutmaßlich auf unterschiedlicher Ernährungsweise und Lebensführung (AlvarezBlasco et al. 2006; Carmina et al. 2003). Es offenbart dennoch die bedeutende Rolle des Übergewichts für die klinische Ausprägung des PCOS. Im Umkehrschluss kann mittels Änderung des Lebensstils und Gewichtsreduktion eine Verbesserung der metabolischen und reproduktiven klinischen Probleme erreicht werden (Clark et al. 1998; Moran et al. 2009).

In jüngster Zeit wird Vitamin D als Co-Faktor des PCOS im Hinblick auf metabolische Störungen diskutiert. Bei 40 bis $70 \%$ der PCOS-Patientinnen kann ein Vitamin-DMangel gefunden werden, welcher mit verschlechterten metabolischen Parametern assoziiert ist (Li et al. 2011; Wehr et al. 2009). Sowohl genetische Polymorphismen des Vitamin-D-Rezeptors als auch Varianten von Vitamin-D-Mangel-assoziierten Genen stehen in Verbindung mit metabolischen Parametern des PCOS (Wehr et al. 2011b). Eine Nahrungsergänzungstherapie mit Vitamin D kann bei PCOSPatientinnen den Glukosehaushalt und Fettstoffwechsel günstig beeinflussen sowie Zyklusstörungen bessern (Wehr et al. 2011a).

\subsubsection{Genetik}

Das PCOS wird ähnlich dem Diabetes mellitus Typ 2 als multifaktoriell bedingte Erkrankung im ätiologischen Kontext von Genetik, Lebenswandel und Umweltfaktoren angesehen (Goodarzi et al. 2011). Gegenstand zahlreicher Studien waren genetische Varianten von Kandidatengenen, welche zum Teil unterschiedlich große Effekte auf den Ausprägungsgrad des PCOS haben. Auf Grund des heterogenen Krankheitsbildes gelang bisher nicht der Nachweis eines einzeln PCOS-auslösenden Gens. Erforscht wurden unter anderem die Genetik der Steroidhormone und Gonadotropine, des Insulins und Zuckerkreislaufs, der 
chronischen Entzündung sowie aktuell der Adipositas durch das FTO-Gen (Prapas et al. 2009; Tan et al. 2010).

Dennoch tritt das PCOS familiär bei erstgradig-verwandten Frauen etwa fünf- bis sechsfach häufiger auf als in der Normalbevölkerung (Kahsar-Miller et al. 2001). Familiäre Häufungen bestehen hinsichtlich gestörter Insulinsekretion (Colilla et al. 2001), androgen verändertem Hormonhaushalt (Legro et al. 1998a; Legro et al. 2002) und Metabolischem Syndrom (Coviello et al. 2009), welche beide auch bei männlichen Verwandten gehäuft auftreten.

Die Rolle intrauterin erhöhter Androgenspiegel im Zusammenhang mit der Entwicklung eines PCOS im Erwachsenenalter ist noch unklar (Goodarzi et al. 2011).

\subsection{Diagnostik}

\subsubsection{Anamnese}

In der Anamnese können bereits die wesentlichen Symptome und Kriterien des PCOS erfasst werden. Neben der allgemeinen Anamnese sollte der Fokus auf Zyklusstörungen mit Oligo-/Amenorrhoe, Behaarungsauffälligkeiten und Hautveränderungen, Kinderwunsch, Fragen zur sexuellen Entwicklung und Familienanamnese sowie auf Gewichtsschwankungen und Medikamenteneinnahme liegen (Weiss et al. 2008).

\subsubsection{Hyperandrogenismus}

Die Untersuchung der klinischen Zeichen einer Hyperandrogenämie berücksichtigt neben einem androgenen Fettverteilungstyp vor allem die Erfassung dermatologischer Zeichen der Hyperandrogenämie: Akne, androgenetische Alopezie, Hirsutismus und Seborrhoe (Lowenstein 2006).

Zur Erfassung und Verlaufskontrolle des Hirsutismus beim PCOS eignen sich verschiedene klinische Scores (Yildiz et al. 2010), wovon die meisten auf dem Score von Ferriman und Gallwey beruhen und nur modifiziert wurden (Ferriman und Gallwey 1961). Je nach verwendetem Score ergeben sich unterschiedliche Cut-OffWerte für einen manifesten Hirsutismus. 
Das Ausmaß der androgenetischen Alopezie kann bei Frauen mittels des von Ludwig entwickelten Scores in drei Graden angegeben werden (Ludwig 1977).

\subsubsection{Polyzystische Ovarien}

Polyzystische Ovarien sind nach den Rotterdam-Kriterien wie folgt definiert: das Vorhandensein von mindestens „12 oder mehr Follikeln in jedem Ovar mit einem Durchmesser von 2 bis $9 \mathrm{~mm}$ und/oder einem erhöhten Ovarvolumen (>10 ml)“ in mindestens einem Ovar (Azziz et al. 2009, S.468; Balen et al. 2003, S. 505).

Als diagnostisches Mittel der Wahl gilt der transvaginale Ultraschall; bei Ablehnung durch die Patientin ist auch der transabdominale Ultraschall des kleinen Beckens möglich. Der optimale Zeitpunkt für die Untersuchung ist regulär in der frühen Follikelphase am dritten bis fünften Zyklustag, im Fall von Oligo-/Amenorrhoe nach Blutungsinduktion (Balen et al. 2003). Unter Einnahme eines Ovulationshemmers können oben genannte Kriterien nicht angewandt werden, da insbesondere orale Ovulationshemmer das Volumen der Ovarien verringern (Christensen et al. 1997).

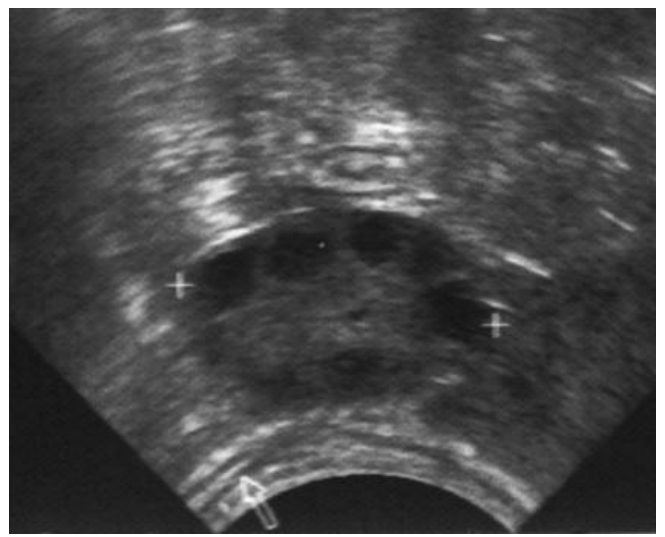

Abbildung 2: Polyzystisches Ovar im transvaginalen Ultraschall. Man erkennt die für das PCOS typische perlschnurartige Morphologie. (Abbildung mit freundlicher Genehmigung von Prof. Dr. Dr. med. B. Hinney, Universitätsfrauenklinik Göttingen)

\subsubsection{Hormondiagnostik}

Wie auch der Ultraschall sollten alle hormonellen Parameter auf Grund physiologischer Schwankungen während des Menstruationszyklus standardmäßig am dritten bis fünften Zyklustag oder nach Blutungsinduktion bei Oligo-/Amenorrhoe bestimmt werden. 
Die hormonelle Basisdiagnostik umfasst folgende Parameter: LH, FSH und Östradiol, Testosteron, SHBG (zur Berechnung des freien Androgenindex), Androstendion und DHEAS, Prolaktin und TSH (Weiss et al. 2008). Mögliche Differentialdiagnosen zuvorderst das late-onset Adrenogenitale Syndrom, ferner unter anderem eine Hyperprolaktinämie, Cushing Erkrankung und hormonbildende Tumoren - sollten mittels weiterer Laborparameter und Testverfahren ausgeschlossen werden.

Folgende Parameter sind beim PCOS erhöht: Gesamt- und freies Testosteron, DHEAS und Androstendion, LH/FSH-Quotient durch LH-Erhöhung. Das SHBG ist beim PCOS typischerweise erniedrigt (Azziz et al. 2009).

In Anbetracht kardiovaskulärer Begleit- und Folgeerkrankungen des PCOS (siehe 1.6) sind Kontrollen entsprechender (Labor-)Parameter des Fettstoffwechsels und inflammatorischer Marker angezeigt (Weiss et al. 2008).

\subsubsection{Insulinresistenz}

In der Forschung gilt die euglykämisch-hyperinsulinämische Clamp-Technik bis heute als der Goldstandard zur exakten Diagnostik der Insulinresistenz (Wallace et al. 2004). Der invasive Test bestimmt direkt diejenige Glukoseinfusionsrate, mit der ein leicht erhöhter, konstanter Blutzucker erreicht wird. Auf Grund der für die Praxis zu aufwändigen Clamp-Technik wurden indirekte Verfahren zur Bestimmung einer Insulinresistenz entwickelt.

Ein solcher indirekter Test ist der Homeostasis Model Assessment-Test (HOMA), welcher lediglich die Nüchternwerte für Glukose und Insulin benötigt (Matthews et al. 1985). Der HOMA ist im Hinblick auf die klinische Praxis eine brauchbare Alternative zur Clamp-Technik (Wallace et al. 2004). Allerdings weist der Test bei jungen, normalgewichtigen PCOS-Patientinnen eine sehr niedrige Sensitivität (41\%) auf. Diese Patientinnen zeigen häufig bloß eine im OGTT stimulierbare Hyperinsulinämie, so dass der HOMA als Test mit Nüchternwerten bei diesen Patientinnen nicht hilfreich ist (Fulghesu et al. 2006). Zur Berechnung der Insulinsensitivität steht der Quantitative insulin sensitivity check index (QUICKI) - eine log-Transformation der Nüchternwerte von Glukose und Insulin - zur Verfügung, welcher gut mit der ClampTechnik korreliert ist (Katz et al. 2000). Beide Verfahren - insbesondere der HOMA eignen sich für den Einsatz im klinischen Alltag mit hoher Anzahl an Patienten. Ein 
weiterer Screening-Test für Insulinresistenz ist der Glukose/Insulin-Quotient, für welchen es ebenfalls beider Nüchternwerte bedarf und welcher nur bei NichtDiabetikern zulässig ist (Legro et al. 1998b).

Der orale Glukosetoleranz-Test (OGTT) ist ein aufwändiger, aber heutzutage zur ausführlichen Diagnostik der Insulinresistenz standardmäßig eingesetzter Test, da allein durch Tests mit Nüchternwerten nicht alle Patientinnen mit Insulinresistenz erfasst werden (Traub 2011). Ein 2-Stunden-OGTT wird allen PCOS-Patientinnen unabhängig vom BMI - bei Erstdiagnostik sowie im Falle normaler Glukosetoleranz alle zwei Jahre empfohlen (Salley et al. 2007).

Trotz aller in Studien belegten Empfehlungen wird die Wahl einer geeigneten Methode zur Diagnostik der Insulinresistenz weiterhin kontrovers diskutiert (Azziz et al. 2009; Traub 2011). Zahlreiche andere indirekte Marker der Insulinresistenz sind noch nicht ausreichend erforscht (Traub 2011).

Die Acanthosis nigricans als dermatologische Manifestation einer Insulinresistenz kann bereits vor labortechnischen Untersuchungen ein diagnostischer Hinweis auf ein PCOS mit Insulinresistenz sein. Die Acanthosis nigricans tritt zusammen mit Hyperandrogenämie und Insulinresistenz als sogenanntes HAIR-AN-Syndrom -Typ A mit Insulinrezeptor-Mutation und Typ B mit Insulinrezeptor-Autoantikörpern - auf, welches als schwere Variante oder Subphänotyp des PCOS betrachtet wird (Lowenstein 2006). Die exakten pathophysiologischen Mechanismen sind noch nicht geklärt. Es wird vermutet, dass es im komplexen Zusammenspiel von erhöhten Insulin- und IGF-1-Spiegeln zu vermehrter Expression von Rezeptoren auf Zellen der Epidermis kommt. Die Folge ist eine irreguläre epidermale Hyperplasie. Patienten mit Acanthosis nigricans profitieren daher von einer Therapie mit Metformin (HermannsLe et al. 2004). Zudem ist diese Hauterscheinung auch hilfreich, um maligne Differentialdiagnosen wie androgenproduzierende Tumoren vom PCOS abzugrenzen (Lowenstein 2006). 


\subsection{Therapie}

\subsection{1 Überblick}

Die Therapie des PCOS sollte nach der im Vordergrund stehenden Symptomatik und individuell auf die Bedürfnisse der Patientinnen ausgerichtet sein. Häufig müssen zugleich mehrere klinische Probleme wie Zyklusstörungen mit oder ohne Infertilität, androgen bedingte Hautsymptome oder metabolische Störungen mit Insulinresistenz behandelt werden. Wenngleich manche therapeutische Mittel (wie Metformin) mehrere Symptome behandeln, ist die Therapie des PCOS selten unimodal (Badawy und Elnashar 2011; Goodarzi et al. 2011).

\subsubsection{Hyperandrogenismus}

Die Therapie von Hirsutismus, Akne und Alopezie besteht aus systemischer und topischer Medikation. Ziele sind die Senkung der Androgenproduktion, eine Reduktion des Anteils an freiem Testosteron (durch Steigerung der SHBGSynthese), die Verminderung der androgenen Wirkung am Endorgan sowie kosmetische Verbesserungen (Goodarzi et al. 2011).

Hirsutismus wird bei Patientinnen ohne Kinderwunsch mit einer Kombination aus oralem Ovulationshemmer (als Östrogen- und Gestagenkombination), einem Antiandrogen sowie mechanischen Methoden behandelt. Empfohlen wird der Beginn mit Ovulationshemmern. Die Östrogenkomponente unterdrückt die LH-vermittelte Androgenproduktion und induziert die SHBG-Synthese. Nach sechs Monaten erfolgloser Therapie sind Antiandrogene wie Spironolacton, Flutamid, Finasterid und Cyproteron-acetat angezeigt. Je nach Indikation können in Einzelfällen auch Glukokortikoide und $\mathrm{GnRH}$-Agonisten eingesetzt werden. Metformin hat indirekt eine therapeutische Wirkung durch Besserung der Hyperinsulinämie (Badawy und Elnashar 2011; Lowenstein 2006).

Die Therapie der Akne basiert ebenso auf oralen Ovulationshemmern und Antiandrogenen sowie zusätzlich auf spezifischer dermatologischer Behandlung wie beispielsweise topischen Antibiotika (Badawy und Elnashar 2011).

Bei androgenetischer Alopezie kommen neben Antiandrogenen und Ovulationshemmern besonders Stimulanzien des Haarwachstums wie Minoxidil in Frage (Goodarzi et al. 2011). 


\subsubsection{Zyklusstörungen und Ovulationsinduktion bei Infertilität}

Vor Einleitung einer medikamentösen Therapie stellen Allgemeinmaßnahmen einen wichtigen Pfeiler in der Therapie von Zyklusstörungen und Infertilität dar. Letztere können sich beispielsweise durch Gewichtsverlust im Zuge normalisierter metabolischer Situation verbessern (Douglas et al. 2006; Moran et al. 2003).

PCOS-Patientinnen ohne Kinderwunsch, die unter Oligo-/Amenorrhoe leiden, profitieren von einer zyklischen oder kontinuierlichen Progesteron-Gabe, welche zudem Schutz vor dem bei PCOS erhöhten Risiko eines Endometriumkarzinoms bietet (Badawy und Elnashar 2011). Meist erfolgt die Gabe in Form eines ÖstrogenGestagen-Ovulationshemmers, worunter sich ein regelmäßiger Zyklus einstellt. Des Weiteren kann Metformin auf indirektem Wege den Zyklus normalisieren und eine Ovulation induzieren (Tang et al. 2010).

Bei Kinderwunsch mit anovulatorischer Infertilität stellt der ÖstrogenrezeptorAntagonist Clomifen-Citrat die First-Line-Therapie zur Induktion einer Ovulation dar. Unter sechsmonatiger Therapie mit Clomifen-Citrat wird eine Geburtsrate von 20 bis $40 \%$ erreicht (Badawy und Elnashar 2011). Metformin ist unter bestimmten Bedingungen eine Alternative, wobei keine Evidenz zur Verbesserung der Geburtsrate unter Metformin allein oder in Kombination mit Clomifen-Citrat besteht (Tang et al. 2010). Glukokortikoide, Gonadotropine und laparoskopische Eingriffe am Ovar werden als Second-Line-Therapie, eine In-vitro-Fertilisation als Third-LineTherapie angesehen (Goodarzi et al. 2011). Ob Aromataseinhibitoren wie Letrozol als Alternative zu Clomifen-Citrat taugen, ist derzeit noch Gegenstand vieler Studien (Badawy et al. 2009; Kamath und George 2011).

\subsubsection{Insulinresistenz}

Die Therapie der Insulinresistenz erfolgt präventiv im Verständnis, dass eine Insulinresistenz im Verlauf Diabetes mellitus und kardiovaskuläre Erkrankungen bedingen kann (siehe Abschnitt 1.6) (Traub 2011).

Der Insulinsensitizer Metformin stellt das wichtigste und das seit langem etablierte Medikament in der Therapie der Insulinresistenz dar. Die komplexen, pharmakologischen Wirkmechanismen sind noch nicht vollständig geklärt. Metformin agiert besonders im hepatischen Stoffwechsel durch eine Vielzahl an 
Enzymreaktionen - unter anderem Hemmung der Glukoneogenese und Lipogenese, Steigerung der Glykolyse und $\beta$-Oxidation. Zudem beeinflusst Metformin den Zuckerund Fettstoffwechsel im Muskel- und Fettgewebe, und nimmt Einfluss auf ovarielle Prozesse (Diamanti-Kandarakis et al. 2010).

Auf Grund unterschiedlicher Definition und Diagnostik der Insulinresistenz sowie des jeweils betrachteten Patientengutes und der Metformindosis wird die Evidenz zur Verbesserung der Insulinresistenz bei PCOS-Patientinnen unter der Therapie mit Metformin unterschiedlich bewertet (Traub 2011). Zahlreiche Studien belegen den Nutzen von Metformin, insbesondere bei übergewichtigen, jugendlichen PCOSPatientinnen und einer Langzeittherapie von 12 bis 24 Monaten (Arslanian et al. 2002; Hahn et al. 2004; Oppelt et al. 2010; Tan et al. 2007). Einzelne Studien zeigten, wiederum mit zum Teil widersprüchlichen Ergebnissen, keine Verbesserung der Insulinresistenz mit kurzzeitiger, drei- bis sechsmonatiger Metformintherapie oder bei jugendlichen PCOS-Patientinnen (Bridger et al. 2006; Otta et al. 2009; Tang et al. 2006).

Für Insulinsensitizer aus der Gruppe der Thiazolidine kann trotz einiger den Nutzen belegender Studien keine allgemeine Therapieempfehlung ausgesprochen werden (Traub 2011).

Darüber hinaus ergeben sich unter Berücksichtigung von Folge- und Nebenerkrankungen des PCOS zum Teil unterschiedliche Empfehlungen. Übergewichtige PCOS-Patientinnen profitieren daher zunächst von einer Änderung des Lebensstils mit Gewichtsreduktion, Diät und Sport (Dunaif 2008; Otta et al. 2009). Metformin ist hierbei die optionale Therapie, wohingegen es bei PCOSPatientinnen mit manifestem Diabetes die First-Line-Therapie darstellt (Dunaif 2008). 


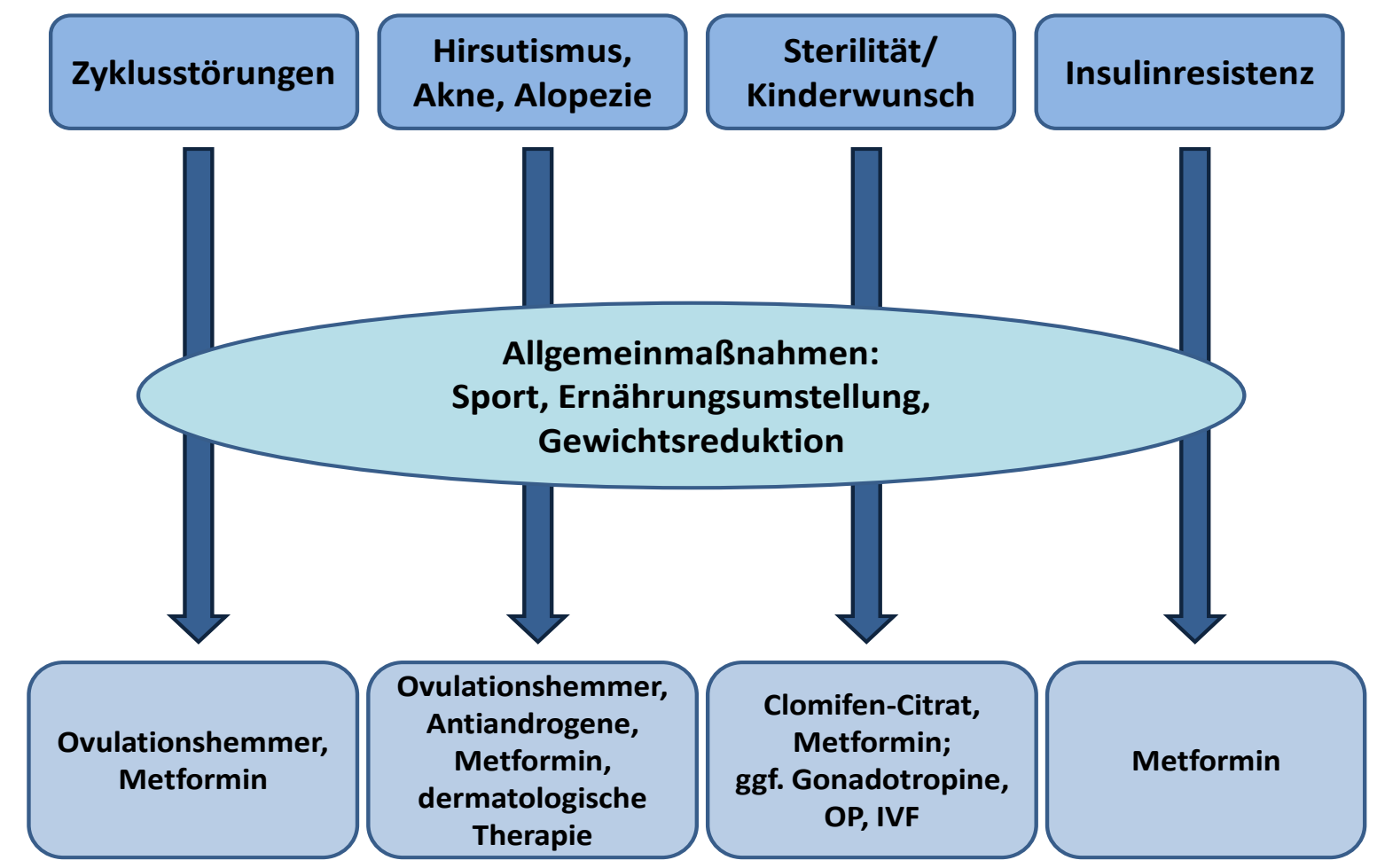

Abbildung 3: Therapieoptionen beim PCOS anhand verschiedener Symptome. (Modifizierte Abbildung mit freundl. Genehmigung von Prof. Dr. Dr. med. B. Hinney, Universitätsfrauenklinik Göttingen)

\subsection{Begleit- und Folgeerkrankungen des PCOS}

\subsubsection{Diabetes mellitus, Metabolisches Syndrom und kardiovaskuläres Risiko}

Patientinnen mit PCOS haben ein erhöhtes Risiko für eine pathologische Glukosetoleranz und einen Diabetes mellitus Typ 2. Eine pathologische Glukosetoleranz und ein im weiteren Verlauf manifester Diabetes mellitus entstehen bei Versagen der kompensatorischen Hypersekretion von Insulin als Reaktion auf eine durch verminderte periphere Insulinsensitivität bedingte Insulinresistenz (Tan et al. 2008). Bei PCOS-Patientinnen besteht eine etwa 2,5-fach erhöhte Prävalenz einer pathologischen Glukosetoleranz. Die Prävalenz des Diabetes mellitus ist 4- bis 4,5-fach erhöht (Moran et al. 2010). Einzelne Studien weisen PCOS-Patientinnen ein fünf- bis zehnfach erhöhtes Risiko für Diabetes mellitus zu. Analog haben Patientinnen im reproduktionsfähigen Alter mit Diabetes mellitus ein erhöhtes Risiko, an einem PCOS zu erkranken (Ovalle und Azziz 2002). 
Unter dem Metabolischen Syndrom versteht man einen Symptomkomplex kardiovaskulärer Risikofaktoren bestehend aus arterieller Hypertonie, stammbetonter Adipositas, Hypertriglyzeridämie, erniedrigtem HDL-Cholesterin und gestörter Glukosetoleranz bei Insulinresistenz, wobei derzeit mehrere Definitionen existieren (Tan et al. 2008). Je nach verwendeter Definition ergeben sich unterschiedlich hohe Prävalenzen des Metabolischen Syndroms bei PCOS-Patientinnen. Eine aktuelle Metaanalyse zeigt eine 2,2- bis 2,9-fach erhöhte Prävalenz im Vergleich zu Patientinnen ohne PCOS (Moran et al. 2010). Bei jüngeren PCOS-Patientinnen mit hohem BMl findet sich besonders häufig ein Metabolisches Syndrom. Dabei wird die Insulinresistenz als der gemeinsame Faktor in der Pathophysiologie betrachtet (Ehrmann et al. 2006).

PCOS-Patientinnen sind von oben genannten kardiovaskulären Risikofaktoren in höherem Maße betroffen (Goodarzi et al. 2011). Postmenopausale PCOSPatientinnen haben vergleichsweise häufiger kardiovaskuläre Ereignisse, wobei es noch an Langzeitstudien mangelt (Rizzo et al. 2009). Das PCOS wird als eine „inflammatorische atherothrombotische Insulinresistenz" betrachtet (Goodarzi et al. 2011, S.224). Diese fördert durch dauerhaft erhöhtes CRP im Sinne einer chronischen Entzündung sowie Endothelschäden mit arteriosklerotischer Verkalkung die Entstehung subklinischer Atherosklerose. Ein Zusammenhang von Adipositas und CRP-Erhöhung wird unterschiedlich bewertet, wobei neueste Metaanalaysen keine Abhängigkeit zeigen (Escobar-Morreale et al. 2011; Tosi et al. 2009). Dennoch sind bei PCOS-Patientinnen kardiovaskuläre Erkrankungen mit erhöhter viszeraler Adipositas korreliert (Cascella et al. 2008). PCOS-Patientinnen haben je nach Phänotyp ein unterschiedliches Risikoprofil, da beispielsweise Patientinnen mit normalem Zyklus und normalen Androgenspiegeln weniger betroffen sind. Darüber hinaus sind noch Faktoren wie Genetik, Umwelt und betrachtete Region zu berücksichtigen (Jovanovic et al. 2009).

\subsubsection{Maligne gynäkologische Tumoren}

Patientinnen mit PCOS haben ein 2,7-fach erhöhtes Risiko, Endometriumkarzinome zu entwickeln (Chittenden et al. 2009). Die hyperplastische Umwandlung des Endometriums als Vorstadium des Karzinoms wird vornehmlich durch die beim 
PCOS typische Anovulation mit stetiger Östrogensekretion ohne Progesteroneinfluss getriggert. Weitere Risikofaktoren wie Adipositas, Infertilität mit Kinderlosigkeit, Hypertonie und Diabetes sind ebenfalls mit dem PCOS assoziiert (Daniilidis und Dinas 2009).

Das Risiko für maligne Tumoren des Ovars ist bei PCOS-Patientinnen 2,5-fach erhöht (Chittenden et al. 2009), insbesondere bei Kinderlosigkeit mit früher Menarche und später Menopause. Die Evidenz ist jedoch umstritten, da Patientinnen mit PCOS durch vergleichsweise weniger Eisprünge per se ein niedrigeres Risikoprofil aufweisen. Mutmaßlich erhöht sich das Risiko im Zuge der Ovulationsinduktion (Daniilidis und Dinas 2009).

Für andere gynäkologische Tumoren einschließlich Brustkrebs liegt noch keine ausreichende Evidenz vor (Chittenden et al. 2009).

\subsubsection{Weitere Erkrankungen und Risiken}

Eine höhere Rate an Spontanaborten nach assistierter Reproduktion bei PCOSPatientinnen wird bei hoher Insulinresistenz beobachtet (Tian et al. 2007). Schwangere PCOS-Patientinnen leiden häufiger an schwangerschaftsinduzierter Hypertonie, Gestationsdiabetes und Präeklampsie sowie neonatalen Komplikationen wie Frühgeburtlichkeit und Intensivpflichtigkeit des Neugeborenen (Boomsma et al. 2006). Eine Empfehlung für Metformin in der Schwangerschaft kann trotz einiger Studien, die einen sicheren Einsatz bei schwangeren PCOS-Patientinnen belegen, derzeit auf Grund fehlender Evidenz in randomisierten Kontrollstudien nicht ausgesprochen werden (Diamanti-Kandarakis et al. 2010).

Untersuchungen zeigen gehäuftes Auftreten der Hashimotothyreoiditis (AIT) bei PCOS-Patientinnen, was auf eine autoimmune Komponente in der Ätiologie des PCOS bzw. einen gemeinsamen genetischen Hintergrund von PCOS und AIT hinweisen könnte (Ganie et al. 2010; Janssen et al. 2004).

Des Weiteren findet sich eine erhöhte Prävalenz an Depression und Angststörungen, wobei Patientinnen mit niedrigerem BMl seltener betroffen sind (Barry et al. 2011). Ein möglicher Zusammenhang von Depression mit den beim PCOS erhöhten CRPWerten wird unterschiedlich bewertet (Benson et al. 2008; Howren et al. 2009). 


\subsection{Fragestellung}

Die vorliegende Dissertation untersucht die Routineversorgung von Patientinnen mit Polyzystischem Ovarsyndrom in einer Praxis für Endokrinologie. Dabei werden thematisch zwei Themenfelder behandelt.

Zunächst ist die Diagnosesicherheit des PCOS unter Praxisbedingungen mit der Frage, wie gut sich in diesem Rahmen PCOS-Patientinnen von Patientinnen mit per Definition ausgeschlossenem PCOS abgrenzen lassen, Gegenstand der Arbeit.

Im zweiten Abschnitt dieser Arbeit folgt die Beurteilung der Therapie einer Insulinresistenz mit Metformin bei PCOS-Patientinnen. Neben der Bewertung des allgemeinen therapeutischen Effekts von Metformin, wird überdies auf die aktuelle Kontroverse zur Wahl eines geeigneten diagnostischen Mittels der Insulinresistenz eingegangen.

Im Lichte der Versorgungsforschung ist das Ziel dieser Arbeit, dass die Ergebnisse eine Konsequenz für die tägliche Arbeit in der endokrinologischen Praxis haben. 


\section{Patienten, Material und Methoden}

\subsection{Studiendesign}

Die vorliegende Dissertation ist eine retrospektive Datenanalyse von 166 Patientinnen, die sich im Zeitraum vom 01.01.2006 bis 31.07.2009 in der Praxis „Endokrinologikum Göttingen“ vorstellten.

\subsection{Patientenauswahl und Gruppeneinteilung}

Zu Beginn der Studie umfasste das anhand der Diagnose in Medistar ${ }^{\circledR}$ gesuchte Kollektiv 230 Patientinnen, wobei sich herausstellte, dass auch Patientinnen ohne OGTT bzw. mit fehlenden (Labor-)Parametern am ehesten anhand einer Ausschlussdiagnose ausgewählt wurden. Nach Bereinigung verblieben noch 166 Patientinnen im Gesamtkollektiv.

Einschlusskriterien waren Frauen jedes Alters bis zum Beginn der Menopause, die sich zumeist aufgrund klinischer Hyperandrogenämiezeichen und/oder Adipositas verschiedenster Ausprägungsgrade vorstellten. Bei diesen Patientinnen wurde nach Ausschluss anderer Erkrankungen ein PCOS diagnostiziert. Als Differentialdiagnosen wurden Untersuchungen für folgende Diagnosen durchgeführt: Cushing-Erkrankung, Hyperprolaktinämie, Tumoren der Nebenniere, Adrenogenitales Syndrom sowie seltene genetisch bedingte Veränderungen. Diese Diagnosen waren ursprünglich als Ausschlusskriterium gedacht.

Im Falle einer im ersten OGTT diagnostizierten Insulinresistenz bei Adipositas im Rahmen des PCOS wurden die Patientinnen nach Ausschluss von Kontraindikationen mit Metformin gewichtsadaptiert behandelt. Die Therapiekontrolle erfolgte nach folgendem Schema:

Nach sechs Wochen erfolgte eine erste Kontrolle der Laborwerte. Nach drei Monaten stellten sich die Patientinnen erstmalig routinemäßig zur Sprechstunde unter der Therapie mit Metformin vor. Nach sechs Monaten war schließlich ein zweiter OGTT zur Therapiekontrolle von Metformin vorgesehen. Dieser konnte in der klinischen Praxis nicht immer termingerecht eingehalten werden, erfolgte jedoch bis spätestens 
zwölf Monate nach Therapiebeginn. Nähere Informationen zum Behandlungsschema mit Metformin sind im Anhang zu finden.

Bei der Analyse der Daten offenbarte sich eine große Heterogenität des Patientenkollektivs, die eine gemeinsame Auswertung als nicht sinnvoll erschienen ließ:

Zum einen stellte sich heraus, dass die Anzahl der Patientinnen mit PCOS geringer als erwartet ausfiel. Des Weiteren hatten nur etwa $30 \%$ aller Patientinnen mit PCOS einen zweiten OGTT. Ein Grund hierfür ist die hohe Abbruchrate der Metformintherapie bei Auftreten von unerwünschten Nebenwirkungen - besonders häufig traten gastrointestinale Beschwerden auf. Bei einigen PCOS-Patientinnen bestanden Kontraindikationen für Metformin, so dass der zweite OGTT nicht durchgeführt wurde. Darüber hinaus führte auch in einigen Fällen eine schlechte Compliance mit nicht wahrgenommenen Terminen zur routinemäßigen Wiedervorstellung im Endokrinologikum zu einer geringeren Rate an zweiten OGTT. Aus all diesen Gründen wurde das Gesamtkollektiv schließlich in fünf Gruppen unterteilt.

\begin{tabular}{|l|l|}
\hline Gruppe 1 & $\begin{array}{l}\text { Gesichertes PCOS } \\
\text { 1. OGTT, Metformin, 2. OGTT } \\
\text { Per Definition kein PCOS, nur Insulinresistenz } 2\end{array}$ \\
Gruppe 3 & $\begin{array}{l}\text { 1. OGTT, Metformin, 2. OGTT } \\
\text { Gesichertes PCOS } \\
\text { 1. OGTT, Metformin, 2. OGTT fehlt (Metformintherapie wegen UAW } \\
\text { vorzeitig beendet) } \\
\text { Gesichertes PCOS }\end{array}$ \\
Gruppe 4 & $\begin{array}{l}\text { 1. OGTT, keine Therapie mit Metformin (bei Kontraindikation, z.B. } \\
\text { eingetretene Schwangerschaft oder abgelehnt) } \\
\text { Per Definition kein PCOS } \\
\text { Keine Insulinresistenz, kein Metformin } \\
\text { Gesichertes PCOS } \\
\text { (1+3+4) }\end{array}$ \\
\hline
\end{tabular}

Tabelle 2: Gruppeneinteilung des Gesamtkollektivs. (Erläuterungen zu den Gruppen 1-5 sowie der neuen Gruppe 6 siehe unten)

Als grobe Einteilung finden sich Patientinnen mit PCOS in den Gruppen 1, 3 und 4 sowie Patientinnen mit ausgeschlossenem PCOS in den Gruppen 2 und 5. 
Patientinnen der Gruppe 1 und 2 wurden wegen ihrer Insulinresistenz mit Metformin behandelt und erhielten während ihrer Kontrollvorstellung einen zweiten OGTT. Dieser fehlte in Gruppe 3, deren Patientinnen die Metformintherapie überwiegend auf Grund unerwünschter Arzneimittelwirkungen vorzeitig beenden mussten. Für die Patientinnen in Gruppe 4 und 5 stellte die Therapie mit Metformin aus verschiedensten Gründen keine Option dar oder wurde abgelehnt.

Die finale Umverteilung der Gruppen entstand nach Rücksprache mit der Abteilung für Medizinische Statistik der Universität Göttingen und Auswertung von Varianzanalysen einzelner Parameter. Auf Grund der zu geringen Anzahl an Patientinnen hätte in Gruppe 2 keine Normalverteilung erreicht werden können. Es ergaben sich bereits erhebliche Varianzunterschiede der Mittelwerte im Parameter Alter, welches im weiteren Kontext der statistischen Auswertung stets zur Verzerrung der Interpretation des Ergebnisses geführt hätte. Auf Anraten der Abteilung für Medizinische Statistik wurde Gruppe 2 vom Gesamtkollektiv ausgeschlossen, und die Gruppen 1,3 und 4 zu einer neuen Gruppe 6 zusammengefasst. Diese neue Gruppe besteht ausschließlich aus Patientinnen mit PCOS und kann für die Auswertung mit Gruppe 5 verglichen werden.

\subsection{Methoden}

\subsubsection{Datengewinnung}

Die Daten stammen aus der Praxis „Endokrinologikum Göttingen“ und lagen in Form elektronischer Patientenakten vor. Die Praxis arbeitet mit den Programmen Medistar® und Moviestar® der Firma MEDISTAR Praxiscomputer GmbH (KarlWiechert-Allee 64, 30625 Hannover).

In der Suchmaske von Medistar ${ }^{\circledR}$ wurde mit den Befehlen „pco“ und „ausschluss+pco“ eine Liste mit 230 Patientinnen generiert. Daten zum OGTT waren in Form eines sogenannten PCO-Arbeitsblattes in Moviestarß hinterlegt. Für die weitere Recherche in Medistar® kamen, wie bereits erwähnt, nur Patientinnen mit einem vollständigen OGTT in Frage. Als Grundlage der Recherche dienten Arztbriefe, Notizen aus Anamnese und Gespräch sowie vorhandene Laboranalysen einschließlich OGTT. 


\subsubsection{Diagnose PCOS}

Das Ärzte-Team des Endokrinologikum Göttingen orientierte sich an den RotterdamKriterien zur Diagnose eines PCOS. In vielen Fällen stellten sich die Patientinnen bereits mit der sonographischen Diagnose der Polyzystischen Ovarien vor. Wenn der sonographische Befund noch nicht vorlag, erfolgten konsiliarische Ultraschalluntersuchungen bei den niedergelassenen Gynäkologen oder auch in der Frauenklinik der Universitätsmedizin Göttingen.

\subsubsection{Diagnostik der Insulinresistenz}

Die Insulinresistenz stellt einen der wichtigsten Faktoren in der Pathophysiologie des PCOS dar. Im Folgenden werden die in der Studie angewandten Methoden zur Ermittlung einer Insulinresistenz näher erläutert.

\subsubsection{Oraler Glukosetoleranztest (OGTT)}

Die Grundlage der Tests zur Ermittlung einer gestörten Glukosetoleranz, Insulinresistenz oder eines bereits manifesten Diabetes mellitus bildete der OGTT. Es ließen sich auch der kinetische Verlauf des Insulinanstiegs und der folgende Blutglukosespiegel erkennen.

Der Test erfolgte in der Regel morgens zu Beginn der Sprechstunde zwischen acht und neun Uhr, nachdem die Patientinnen mindestens zwölf Stunden Nahrungskarenz einzuhalten hatten. Die Bestimmung der Glukosewerte erfolgte im Endokrinologikum aus kapillärem Vollblut aus dem Ohr. Für die Bestimmung der Insulin- und C-PeptidWerte wurde den Patientinnen venöses Vollblut entnommen und zur Analyse in das Zentrallabor des Endokrinologikum Hamburg geschickt. Nach Abnahme der Nüchternwerte wurden die Patientinnen gebeten, innerhalb von fünf Minuten die in $300 \mathrm{ml}$ Wasser aufgelösten $75 \mathrm{~g}$ Glukose (Dextro-O.G.T.®, Firma Roche) zu trinken. Nach 60 und 120 Minuten erfolgten weitere Blutentnahmen. 


\begin{tabular}{|c|c|c|c|c|}
\hline & $\begin{array}{c}\text { Normale } \\
\text { Glukosetoleranz }\end{array}$ & $\begin{array}{c}\text { Abnorme } \\
\text { Nüchternglukose }\end{array}$ & $\begin{array}{c}\text { Gestörte } \\
\text { Glukosetoleranz }\end{array}$ & $\begin{array}{c}\text { Diabetes } \\
\text { mellitus }\end{array}$ \\
\hline $\begin{array}{c}\text { Glukose } \\
\text { nüchtern } \\
\text { (mg/dl) }\end{array}$ & $>90$ & $90-109$ & $>110$ & $\geq 110$ \\
\hline $\begin{array}{c}\text { Glukose } \\
\mathbf{2} \text { h } \\
(\mathbf{m g} / \mathbf{d l})\end{array}$ & $>140$ & - & $\underline{\text { und }}$ & $\underline{\text { und/oder }}$ \\
\hline
\end{tabular}

Tabelle 3: Referenzwerte im OGTT für Vollblutglukose kapillär. Einteilung nach der Leitlinie "Definition, Klassifikation und Diagnostik des Diabetes mellitus“ der Deutschen Diabetes Gesellschaft. (http://www.deutsche-diabetes-gesellschaft.de/redaktion/mitteilungen/leitlinien/EBL_Klassifikation_ Update_2004.pdf, Stand 07.09.2011)

Zudem konnte mit dem OGTT auch die Gesamtsekretion des Insulins als Maß für die bei Insulinresistenz resultierende Hyperinsulinämie errechnet werden: die Fläche unter der Kurve (area under the curve, AUC) von Insulin. Dies galt ebenfalls für die Ausschüttung von Glukose (AUC Glukose) und des C-Peptids (AUC C-Peptid).

\subsubsection{Homeostasis Model Assessment-Test (HOMA)}

HOMA bestimmt die Insulinresistenz auf indirektem Wege, welcher lediglich die Nüchternwerte für Glukose und Insulin benötigt.

Das Ergebnis des HOMA wird auch als HOMA Index oder HOMA-IR (=InsulinResistenz) angegeben. Der HOMA Index wurde mit der folgenden Formel berechnet:

HOMA IR $=[($ Nüchterninsulin $\mu U / m I \times$ Nüchternglukose mg/dl) $/ 22,5 / 18]$

\begin{tabular}{|c|l|}
\hline HOMA-IR & \multicolumn{1}{|c|}{ Interpretation } \\
\hline$<2,5$ & Normwert \\
$>2,5$ & Insulinresistenz möglich \\
$>5,0$ & Durchschnittswert bei Diabetes mellitus Typ 2 \\
\hline
\end{tabular}

Tabelle 4: Interpretation des HOMA-IR. 


\subsubsection{Quantitative insulin sensitivity check index (QUICKI)}

Werte des QUICKI errechneten sich aus folgender Formel:

$$
\text { QUICKI = } 1 \text { / [log Nüchterninsulin } \mu U / m I+\log \text { Nüchternglukose mg/dl] }
$$

Die Spannweite der Werte erstreckt sich von 0,45 für gesunde Menschen bis 0,30 für Diabetiker, so dass ein niedrigerer Wert eine höhere Insulinresistenz bedeutet.

\subsubsection{Klinische Hyperandrogenämie}

Zur Erfassung des Hirsutismus wurde der Ferriman-Gallwey-Score verwendet. Der Ferriman-Gallwey-Score betrachtet neun Hautareale mit einem Punktesystem von null bis vier, wobei manifester Hirsutismus ab einem Wert von fünf Punkten besteht (siehe Anhang). Nicht bei jeder Patientin war der Ferriman-Gallwey-Score in der elektronischen Patientenakte vermerkt. Daher wurden auch Arztbriefe und Anamnesebögen mit berücksichtigt, so dass schlussendlich nur ein nominales Bewertungssystem des Hirsutismus - ja oder nein - mit in die Auswertung gebracht wurde.

Zur Erfassung der Akne wurde kein spezielles klinisches Instrument herangezogen. $\mathrm{Ob}$ eine Akne vorlag, wurde in Arztbriefen und/oder Anamnesebögen vermerkt. Wiederum wurde ein nominales Bewertungssystem mit in die Auswertung gebracht.

Das Ausmaß einer androgenetisch bedingten Alopezie wurde nicht standardmäßig untersucht, sondern lediglich in Einzelfällen im Arztbrief vermerkt.

\subsubsection{Laboruntersuchungen}

Der auf den nächsten zwei Seiten folgenden Tabelle sind die verwendeten Laboruntersuchungen zu entnehmen: 


\begin{tabular}{|c|c|c|c|c|c|}
\hline \multirow[t]{2}{*}{ Testname } & \multirow[t]{2}{*}{ Einheit } & \multicolumn{2}{|c|}{ Referenzbereich } & \multirow[t]{2}{*}{ Methode } & \multirow[t]{2}{*}{ Hersteller } \\
\hline & & Frauen & Männer & & \\
\hline Androstendion & $\mathrm{ng} / \mathrm{ml}$ & $0,5-2,7$ & $0,5-2,8$ & RIA & DSL \\
\hline Cholesterin & $\mathrm{mg} / \mathrm{dl}$ & \multicolumn{2}{|c|}{$\begin{array}{c}\text { Wünschenswert: }<200 \\
\text { Grenzwertig erhöht: } 200-239 \\
\text { Hoch: } \geq 200\end{array}$} & $\begin{array}{l}\text { CHOD- } \\
\text { PAP }\end{array}$ & Roche \\
\hline C-Peptid basal & $\mu \mathrm{g} / \mathrm{l}$ & \multicolumn{2}{|c|}{$0,8-4,2$} & CLIA & DiaSorin \\
\hline DHEA-Sulfat & $\mu \mathrm{g} / \mathrm{ml}$ & $0,4-4,3$ & $0,8-5,6$ & CLIA & Siemens \\
\hline $\begin{array}{l}\text { 17-beta-Estradiol } \\
\text { (E2) }\end{array}$ & $\mathrm{pg} / \mathrm{ml}$ & $\begin{array}{c}\text { Zyklusphase: } \\
\text { Follikel: } 12,5-166,0 \\
\text { Ovulation: } 85,8-498,0 \\
\text { Luteal: } 43,8-211,0 \\
\text { Postmenopausal: } \\
<5,0-54,7\end{array}$ & $<45,0$ & ECL & Roche \\
\hline $\begin{array}{c}\text { FAl (freier } \\
\text { Androgenindex) }\end{array}$ & - & $<3,5$ & $15,0-95,0$ & $\begin{array}{c}\text { Berechnet: } \\
\text { Testosteron } \\
(\mathrm{nmol} / \mathrm{l}) \mathrm{x} \\
100 / \mathrm{SHBG} \\
(\mathrm{nmol} / \mathrm{l})\end{array}$ & $\begin{array}{l}\text { Siehe } \\
\text { Testosteron } \\
\text { und SHBG }\end{array}$ \\
\hline FSH & $\mathrm{mlU} / \mathrm{ml}$ & $\begin{array}{c}\text { Zyklusphase: } \\
\text { Follikel: } 3,5-12,5 \\
\text { Ovulation: } 4,7-21,5 \\
\text { Luteal: } 1,7-7,7 \\
\text { Postmenopausal: } \\
\text { 25,8-134,8 }\end{array}$ & $\begin{array}{l}<40 \text { Jahre: }<6,0 \\
>40 \text { Jahre: }<13,0\end{array}$ & ECL & Roche \\
\hline $\begin{array}{l}\text { Glukose nüchtern } \\
\text { (im Blut) }\end{array}$ & $\mathrm{mg} / \mathrm{dl}$ & \multicolumn{2}{|c|}{$75-110$} & PHOT & Roche \\
\hline HDL-Cholesterin & $\mathrm{mg} / \mathrm{dl}$ & $\begin{array}{c}\text { Risiko: } \\
\text { Kein: }>65,0 \\
\text { Mäßig: } 45,0-65,0 \\
\text { Hoch: }<45,0\end{array}$ & $\begin{array}{c}\text { Risiko: } \\
\text { Kein: }>55,0 \\
\text { Mäßig: } 35,0-55,0 \\
\text { Hoch: }<35,0\end{array}$ & $\begin{array}{l}\text { Homo- } \\
\text { gener } \\
\text { enzyma- } \\
\text { tischer } \\
\text { Farbtest }\end{array}$ & Roche \\
\hline Insulin basal & $\mathrm{mlE} / \mathrm{l}$ & \multicolumn{2}{|c|}{$3,2-16,3$} & CLIA & DiaSorin \\
\hline LDL-Cholesterin & $\mathrm{mg} / \mathrm{dl}$ & \multicolumn{2}{|c|}{$\begin{array}{l}\text { KHK und D. mellitus: }<100,0 \\
\geq 2 \text { Risikofaktoren: }<130,0 \\
\leq 2 \text { Risikofaktoren: }<160,0\end{array}$} & $\begin{array}{c}\text { Berechnet } \\
\text { nach } \\
\text { Friedewald } \\
\text { formel (bei } \\
\text { TRIG<400 } \\
\text { mg/dl) }\end{array}$ & Roche \\
\hline Leukozyten & $\mathrm{x} 1000 / \mu \mathrm{l}$ & \multicolumn{2}{|c|}{ >12 Jahre: 4,6-10,2 } & $\begin{array}{l}\text { Masch. } \\
\text { Zählung }\end{array}$ & Sysmex \\
\hline Lutropin/LH & $\mathrm{mlU} / \mathrm{ml}$ & $\begin{array}{c}\text { Zyklusphase: } \\
\text { Follikel: 1,9-12,5 } \\
\text { Ovulation: } 8,7-76,3 \\
\text { Luteal: 0,5-16,9 } \\
\text { Postmenopausal: } \\
\text { 15,9-54,0 }\end{array}$ & $1,7-8,6$ & ECL & Roche \\
\hline \multirow{2}{*}{$\begin{array}{l}\text { 17-OH- } \\
\text { Progesteron } \\
\text { basal }\end{array}$} & \multirow{2}{*}{$\mu \mathrm{g} / \mathrm{l}$} & \multicolumn{2}{|c|}{ Kinder (1-13 Jahre): 0,1-1,4 } & RIA & DSL \\
\hline & & Follikelphase: $0,3-1,0$ & $0,9-3,1$ & & \\
\hline
\end{tabular}




\begin{tabular}{|c|c|c|c|c|c|}
\hline Progesteron & $\mathrm{ng} / \mathrm{ml}$ & $\begin{array}{c}\text { Zyklusphase: } \\
\text { Follikel: } 0,2-1,5 \\
\text { Ovulation: } 0,8-3,0 \\
\text { Luteal: } 1,7-27,0 \\
\text { Postmenopausal: } \\
0,1-0,8\end{array}$ & $0,2-1,4$ & ECL & Roche \\
\hline SHBG & $\mathrm{nmol} / \mathrm{l}$ & $18,0-144,0$ & $10,0-57,0$ & CLIA & Siemens \\
\hline $\begin{array}{c}\text { Testosteron } \\
\text { gesamt }\end{array}$ & $\mathrm{ng} / \mathrm{ml}$ & $0,06-0,82$ & $2,8-8,0$ & ECLIA & $\begin{array}{c}\text { Roche } \\
\text { Diagnostics }\end{array}$ \\
\hline $\begin{array}{l}\text { Anti-TPO } \\
\text { (Thyreoper- } \\
\text { oxidase } \\
\text { Antikörper) }\end{array}$ & $\mathrm{IU} / \mathrm{ml}$ & \multicolumn{2}{|c|}{$<35,00$} & ECLIA & Roche \\
\hline $\begin{array}{c}\text { TRAK (TSH- } \\
\text { Rezeptor- } \\
\text { Autoantikörper) }\end{array}$ & $\mathrm{U} / \mathrm{I}$ & \multicolumn{2}{|c|}{ Negativ: $<9,0$} & RIA & $\begin{array}{c}\text { Thermo } \\
\text { SCIENTIFIC }\end{array}$ \\
\hline Triglyzeride & $\mathrm{mg} / \mathrm{dl}$ & \multicolumn{2}{|c|}{$<200,0$} & $\begin{array}{l}\text { Enzymati- } \\
\text { scher } \\
\text { Farbtest }\end{array}$ & Roche \\
\hline TSH & $\mathrm{mlU} / \mathrm{l}$ & \multicolumn{2}{|c|}{$0,27-2,5$} & ECLIA & Roche \\
\hline
\end{tabular}

Tabelle 5: Laboruntersuchungen in dieser Arbeit.

\subsubsection{Nebendiagnosen}

Die Diagnose einer Hashimoto-Thyreoiditis erfolgte anhand der Sonographie mit Größe und Echogenität der Schilddrüse sowie der Werte von TSH und den TPOAntikörpern.

\subsubsection{Statistik}

Die statistische Auswertung dieser Dissertation wurde mit freundlicher Unterstützung der Abteilung für Medizinische Statistik der Universitätsmedizin Göttingen durchgeführt.

Als statistisches Auswertungsprogramm wurde STATISTICA 9.0 für Windows XP der Firma StatSoft verwendet.

Die deskriptiven Ergebnisse wurden als arithmetisches Mittel (MW) mit einer Standardabweichung (SD) und dem Median dargestellt. Ein signifikantes Ergebnis lag bei einem $p$-Wert $\leq 0,05$ vor; hochsignifikante Ergebnisse bei einem $p$-Wert $\leq 0,01$. Beide wurden in Grafiken mit einem bzw. zwei Sternchen - oder drei Sternchen bei $p<0,001$ - gekennzeichnet. Ein $p$-Wert $\leq 0,1$ wurde als Tendenz gewertet. 
Um die Signifikanz von Mittelwertdifferenzen des ursprünglichen Kollektivs mit fünf Gruppen zu testen, wurde das Varianzanalyseverfahren ANOVA angewandt.

Der Vergleich des neuen Kollektivs mit nur noch zwei Gruppen erfolgte bei parametrischen Daten mit dem zweiseitigen t-Test für unabhängige Stichproben bzw. mit dem Pearson-Chi-Quadrat-Test für nicht-parametrische Daten.

Der Vergleich zweier Zeitpunkte der Gruppe PCOS-Met wurde im Falle parametrischer Daten mit dem $\mathrm{t}$-Test für gepaarte Stichproben ausgewertet; bei nicht-parametrischen Daten wurde der Wilcoxon-Test für gepaarte Stichproben angewandt.

Der Einfluss eines Ovulationshemmers auf die Gruppen PCOS und Ausschluss PCOS bzw. auf beide Untersuchungszeitpunkte der Gruppe PCOS-Met wurde bei metrischen Daten mittels zweiseitigem t-Test für unabhängige Stichproben bzw. mittels Pearson-Chi-Quadrat-Test für nicht-parametrische Daten durchgeführt.

Der Zusammenhang einzelner Parameter wurde graphisch durch die Auftragung einer Korrelationsgeraden dargestellt mit Angabe des Korrelationskoeffizienten $r$ nach Pearson, des Bestimmtheitsmaßes $r^{2}$ und des $p$-Wertes. Diese Werte wurden in Statistica 9.0 berrechnet. 


\section{Ergebnisse}

\subsection{Hinweise zur Patientencharakterisierung}

Die nachfolgende Patientencharakterisierung orientiert sich an der in Abschnitt 2.2 (S. 19) erläuterten Gruppeneinteilung des Gesamtkollektivs.

Zunächst werden die klinischen Basisparameter des ursprünglichen Kollektivs mit den Gruppen 1 bis 5 dargestellt. Im Anschluss daran folgt der Vergleich der neu zusammengefassten Gruppe PCOS (6) mit der Gruppe Ausschluss PCOS (5). Der betrachtete Untersuchungszeitpunkt ist hierbei stets die Erstuntersuchung im Endokrinologikum. Es werden daher die Parameter nur zu einem Zeitpunkt verglichen, was - um Redundanzen zu vermeiden - fortan nicht mehr erwähnt wird.

Im zweiten Abschnitt wird durch den Vergleich zweier Untersuchungszeitpunkte in der Gruppe PCOS-Met (1) auf die spezielle Fragestellung des therapeutischen Effekts von Metformin eingegangen.

\subsection{Charakterisierung der Gruppen 1-5}

\subsubsection{Klinische Basisparameter}

Es ergaben sich Varianzunterschiede bei Alter und BMI.

\begin{tabular}{|c|c|c|c|c|c|c|c|}
\hline Grt & $\begin{array}{l}\text { Gesamt- } \\
\text { kollektiv }\end{array}$ & $\begin{array}{l}(1) \\
\text { PCOS- } \\
\text { Met }\end{array}$ & $\begin{array}{c}\text { (2) } \\
\text { IR-Met }\end{array}$ & $\begin{array}{c}(3) \\
\text { PCOS- } \\
\text { Met } \\
\varnothing \\
2.0 \mathrm{GTT}\end{array}$ & $\begin{array}{c}(4) \\
\text { PCOS } \\
\varnothing \text { Met }\end{array}$ & $\begin{array}{c}\text { (5) } \\
\text { Ausschl. } \\
\text { PCOS }\end{array}$ & p-Wert \\
\hline Anzahl (n) & 166 & 30 & 6 & 35 & 37 & 58 & \\
\hline $\begin{array}{c}\text { Alter } \\
\text { (Jahre) }\end{array}$ & $\begin{array}{c}29,81 \\
\pm 10,22\end{array}$ & $\begin{array}{l}27,37 \\
\pm 8,61\end{array}$ & $\begin{array}{c}42,17 \\
\pm 14,05\end{array}$ & $\begin{array}{r}27,86 \\
\pm 8,31\end{array}$ & $\begin{array}{l}25,59 \\
\pm 7,17\end{array}$ & $\begin{array}{c}33,67 \\
\pm 11,19\end{array}$ & 0,00001 \\
\hline $\begin{array}{c}\text { Gewicht } \\
\text { (kg) }\end{array}$ & $\begin{array}{c}86,50 \\
\pm 24,52\end{array}$ & $\begin{array}{c}91,62 \\
\pm 15,29\end{array}$ & $\begin{array}{r}101,70 \\
\pm 37,45\end{array}$ & $\begin{array}{c}91,44 \\
\pm 26,29\end{array}$ & $\begin{array}{c}82,52 \\
\pm 22,24\end{array}$ & $\begin{array}{c}81,84 \\
\pm 26,24\end{array}$ & 0,30 \\
\hline $\begin{array}{c}\text { Größe } \\
(\mathrm{m})\end{array}$ & $\begin{array}{r}1,65 \\
\pm 0,06\end{array}$ & $\begin{array}{r}1,65 \\
\pm 0,07\end{array}$ & $\begin{array}{c}1,63 \\
\pm 0,06\end{array}$ & $\begin{array}{r}1,66 \\
\pm 0,07\end{array}$ & $\begin{array}{c}1,64 \\
\pm 0,05\end{array}$ & $\begin{array}{r}1,65 \\
\pm 0,05\end{array}$ & 0,65 \\
\hline BMI $\left(\mathrm{kg} / \mathrm{m}^{2}\right)$ & $\begin{array}{l}31,67 \\
\pm 8,21\end{array}$ & $\begin{array}{r}33,70 \\
\pm 5,13\end{array}$ & $\begin{array}{c}37,73 \\
\pm 11,66\end{array}$ & $\begin{array}{c}37,73 \\
\pm 11,66\end{array}$ & $\begin{array}{l}30,55 \\
\pm 7,79\end{array}$ & $\begin{array}{r}29,92 \\
\pm 8,79\end{array}$ & 0,0511 \\
\hline
\end{tabular}

Tabelle 6: Klinische Basisparameter. Die Tabelle zeigt die Varianzunterschiede von Gruppe 2 im Vergleich zu allen anderen Gruppen insbesondere hinsichtlich Alter und BMI. (Abkürzungen: Met=Metformin, IR=Insulinresistenz, Ausschl.=Ausschluss) 


\subsubsection{Alter}

Die Patientinnen des Gesamtkollektivs waren zum ersten Untersuchungszeitpunkt zwischen 13 und 63 Jahre alt, der Median lag bei 28 Jahren. Die mit Hilfe des Verfahrens ANOVA analysierten Altersmittelwerte wiesen eine sehr hohe Signifikanz auf $(p=0,00001)$. Im Vergleich zu allen anderen Gruppen waren die Patientinnen der Gruppe 2 signifikant älter (Median: 43,5 Jahre).

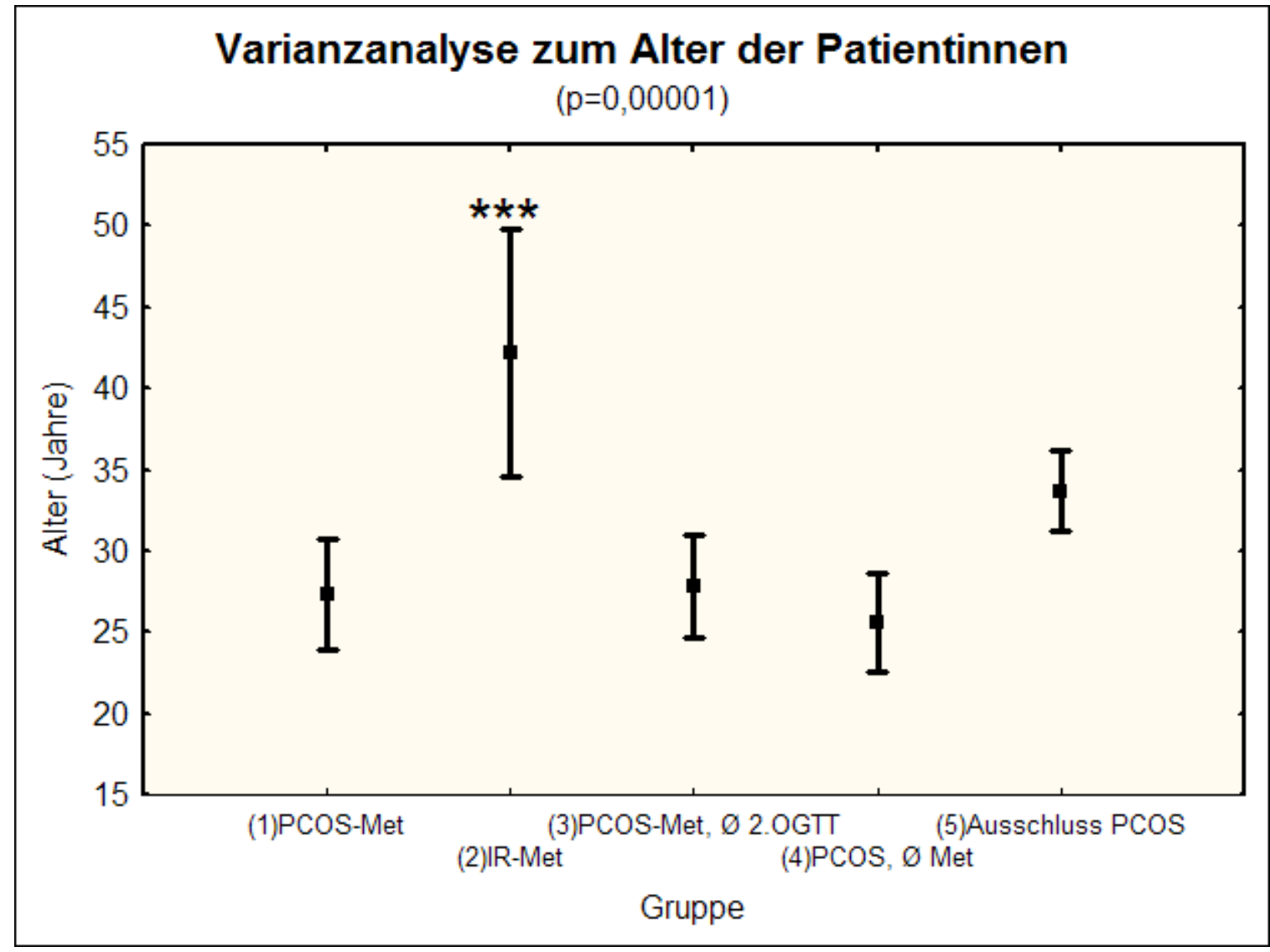

Abbildung 4: Varianzanalyse der Altersmittelwerte in den Gruppen 1 bis 5 . Die vertikalen Balken zeigen das $95 \%$-Konfidenzintervall. Hochsignifikante $p$-Werte $(<0,001)$ wurden mit drei Sternchen gekennzeichnet. (Abkürzungen: Met=Metformin, IR=Insulinresistenz)

\subsubsection{BMI}

Als weiterer klinischer Basisparameter wurde der BMI betrachtet, welcher ebenfalls durch eine große Spannweite auffiel. Die Patientinnen im Gesamtkollektiv wiesen BMI-Werte von 16,9 bis in Einzelfällen auch $62 \mathrm{~kg} / \mathrm{m}^{2}$ auf (Median: $31,1 \mathrm{~kg} / \mathrm{m}^{2}$ ). 

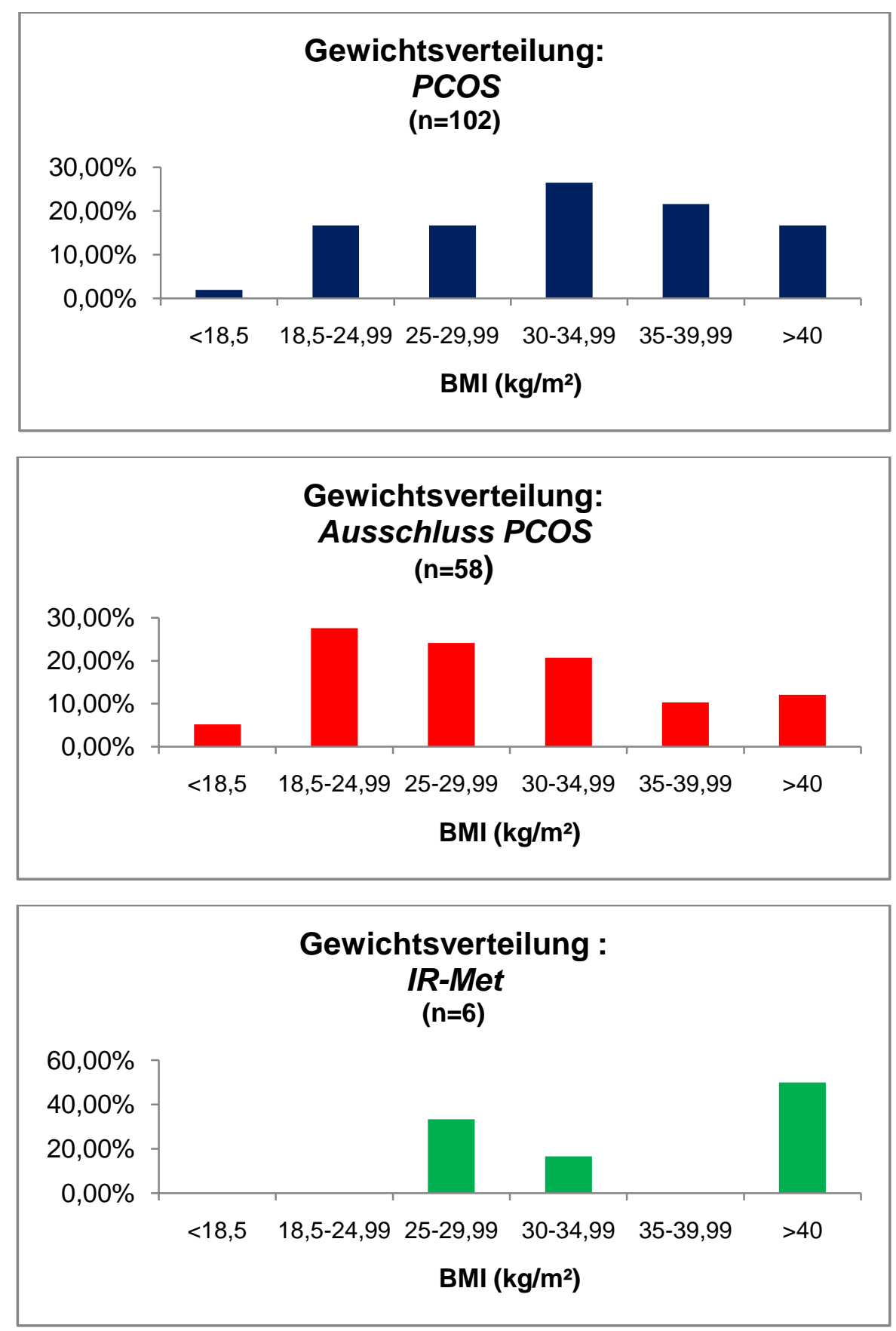

Abbildungen 5, 6 und 7: Darstellung des Körpergewichts anhand des BMI. Die BMI-Einteilung erfolgt gemäß aktuellem WHO-Bericht (WHO 2000). Der BMI [in der Einheit kg/m²] definiert zudem die Begriffe Unter-, Normal- und Übergewicht sowie den Grad der Adipositas. (Untergewicht: BML 18,5 . Normalgewicht: $B M I=18,5-24,99$. Übergewicht/Präadipositas: $B M I=25-29,99$. Adipositas Grad I: BMI=30-34,99. Adipositas Grad II: BMI=35-39,99. Adipositas Grad III: BMI $\geq 40$.)

Die Abbildungen veranschaulichen, dass rund drei Viertel aller Patientinnen (77,1\%) einen $\mathrm{BMI} \geq 25 \mathrm{~kg} / \mathrm{m}^{2}$ hatten und somit per Definition nicht mehr normalgewichtig waren. Des Weiteren erkennt man, dass PCOS-Patientinnen im Vergleich stärker zu 
Adipositas $\left(\mathrm{BMl} \geq 30 \mathrm{~kg} / \mathrm{m}^{2}\right)$ neigten $(64,7 \%)$ als Patientinnen mit ausgeschlossenem $\operatorname{PCOS}(43,1 \%)$.

Die Analyse mit ANOVA ergab keine Signifikanz innerhalb der fünf Gruppen bezüglich des BMI-Mittelwertes, allerdings eine Tendenz $(p=0,0511)$. Die sechs Patientinnen der Gruppe IR-Met (2) hatten im Vergleich zu den restlichen Gruppen in der Tendenz einen höheren BMI (Median: 36,88 kg/m²).

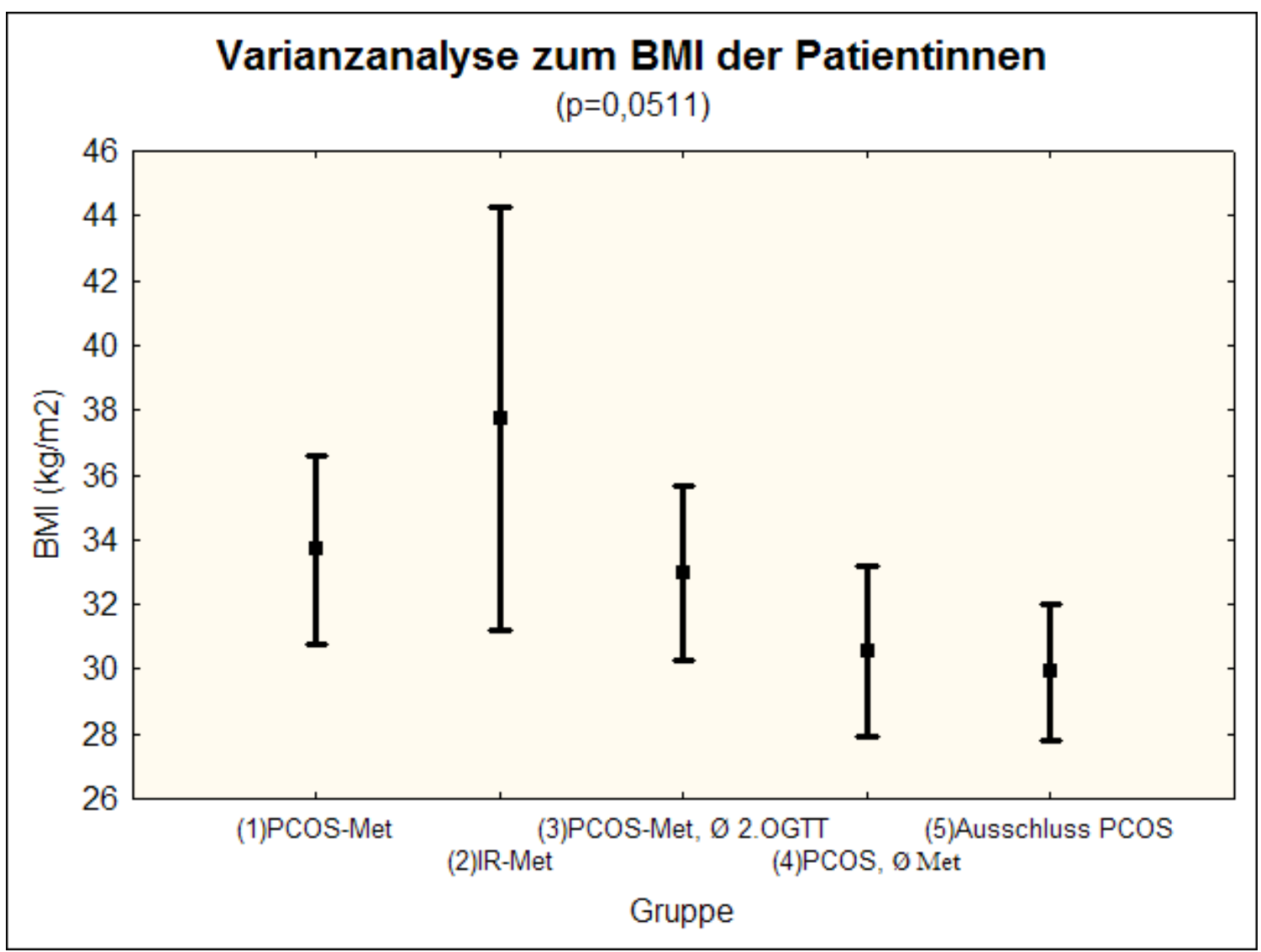

Abbildung 8: Varianzanalyse der BMI-Mittelwerte in den Gruppen 1 bis 5. Die vertikalen Balken zeigen das 95\%-Konfidenzintervall. (Abkürzungen: Met=Metformin, IR=Insulinresistenz) 


\subsection{Patientinnencharakterisierung nach Gruppenzusammenfassung}

\subsubsection{Gesamtkollektiv nach endgültiger Gruppenverteilung}

Nach Ausschluss von Gruppe IR-Met (2) umfasste das neue Gesamtkollektiv noch 160 Patientinnen, bestehend aus der neuen Gruppe PCOS mit 102 PCOSPatientinnen und der Gruppe Ausschluss PCOS mit 58 Patientinnen.
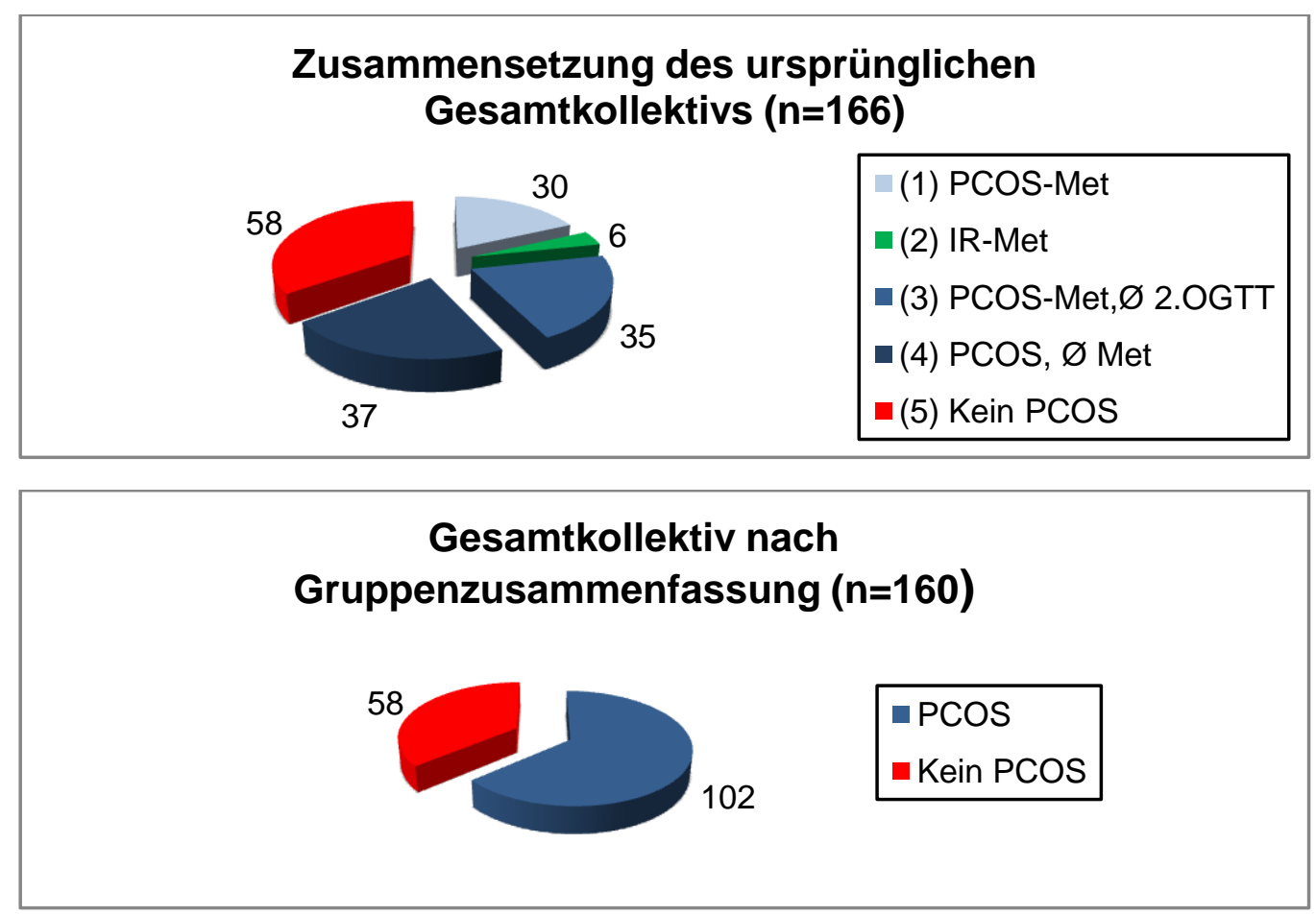

Abbildungen 9 und 10: Gruppen des ursprünglichen Gesamtkollektivs und nach Gruppenzusammenfassung.

\subsubsection{Klinische Basisparameter}

Ähnlich der Darstellung in 3.2 werden zunächst die klinischen Basisparameter Alter, Gewicht, Größe und BMI im neuen Gesamtkollektiv behandelt.

\begin{tabular}{|c|ccc|}
\hline Parameter & Anzahl $(\mathbf{n})$ & MW \pm SD & Median \\
\hline Alter (Jahre) & 160 & $29,35 \pm 9,81$ & 27,50 \\
Gewicht $(\mathbf{k g})$ & 160 & $85,93 \pm 23,89$ & 84,35 \\
Größe (m) & 160 & $1,65 \pm 0,06$ & 1,65 \\
BMI $\left(\mathbf{k g} / \mathbf{m}^{2}\right)$ & 160 & $31,44 \pm 8,02$ & 31,02 \\
\hline
\end{tabular}

Tabelle 7: Klinische Basisparameter des neuen Gesamtkollektivs. 
Die zusammengefasste Gruppe PCOS hatte mit 63,75 \% den größeren Anteil am neuen Gesamtkollektiv. In der Gruppe der Patientinnen mit ausgeschlossenem PCOS hatten sich keine Änderungen ergeben, so dass zur besseren Übersicht die Daten aus 3.2.1 erneut dargestellt werden.

\begin{tabular}{|l|ccc|ccc|c|}
\hline & \multicolumn{3}{|c}{ PCOS } & \multicolumn{3}{|c|}{ Ausschluss PCOS } & p-Wert \\
Parameter & $\begin{array}{c}\text { Anzahl } \\
\text { (n) }\end{array}$ & $\begin{array}{c}\text { MW } \\
\mathbf{\pm} \text { SD }\end{array}$ & Median & Anzahl & MW & Median & \\
\hline Alter (J) & 102 & $26,89 \pm 7,99$ & 24,50 & 58 & $33,67 \pm 11,19$ & 34,50 & 0,0001 \\
Gewicht kg) & 102 & $88,26 \pm 22,25$ & 87,15 & 58 & $81,84 \pm 26,24$ & 75,00 & n.s. \\
Größe (m) & 102 & $1,65 \pm 0,06$ & 1,65 & 58 & $1,65 \pm 0,05$ & 1,65 & n.s. \\
BMI (kg/m $\left.{ }^{2}\right)$ & 102 & $32,31 \pm 7,45$ & 32,04 & 58 & $29,92 \pm 8,79$ & 28,96 & 0,0835 \\
\hline
\end{tabular}

Tabelle 8: Klinische Basisparameter der beiden Gruppen.

\subsubsection{Alter}

Der auffällige Altersunterschied zwischen beiden Gruppen erwies sich im zweiseitigen t-Test für unabhängige Stichproben als hochsignifikant $(p=0,0001)$.

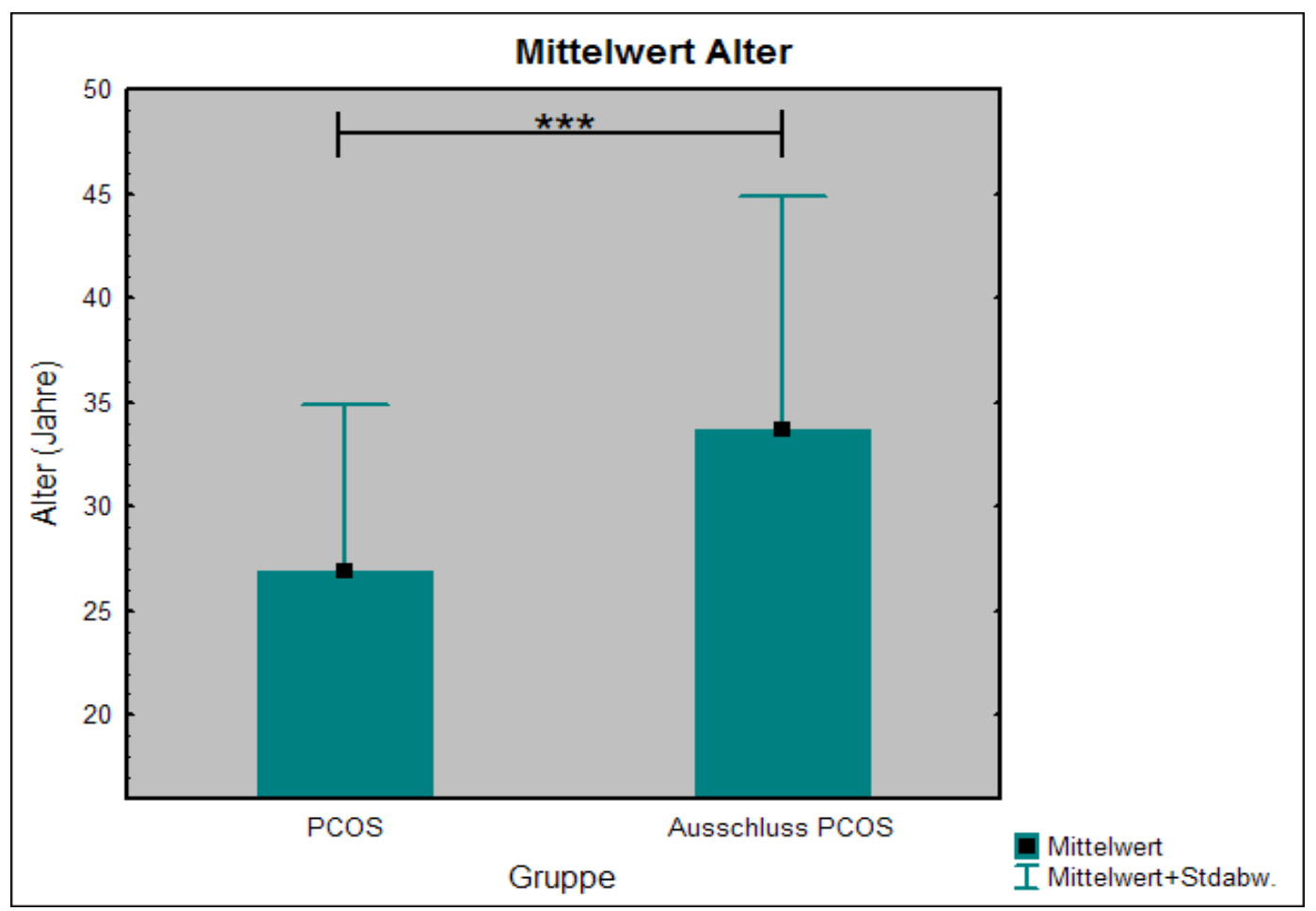

Abbildung 11: Darstellung der Mittelwerte des Alters in beiden Gruppen $(p=0,0001)$. Die PCOSPatientinnen waren im Durchschnitt knapp 7 Jahre jünger. 


\subsubsection{BMI}

Demgegenüber erwies sich der Parameter $B M I$ als nicht signifikant, aber als Tendenz ( $p=0,0835)$. Wie jedoch bereits in 3.2.1.2 dargestellt wurde, neigten PCOSPatientinnen vermehrt zu Adipositas. Bei einer nachträglichen Auswertung zeigte sich der BMI von der Einnahme eines Ovulationshemmers statistisch nicht beeinflusst, so dass die Auswertung der jeweils gesamten Gruppe valide war.

\subsubsection{Klinische PCOS-Parameter}

Die bei Anamnese und körperlicher Untersuchung erhobenen klinischen Parameter Akne, Hirsutismus und Zyklusstörungen wurden im Kontext der Pathophysiologie des PCOS gesondert betrachtet. Im Falle eines signifikanten Unterschieds bei Einnahme eines Ovulationshemmers wurden Patientinnen mit Ovulationshemmer in der Vormedikation von der Auswertung ausgeschlossen. Da es sich um nichtparametrische Daten handelt, wurde der Pearson-Chi-Quadrat-Test angewandt.

\subsubsection{Akne}

In beiden Gruppen hatte die Einnahme eines Ovulationshemmers keinen statistisch relevanten Einfluss auf das Auftreten von Akne, so dass alle Patientinnen ausgewertet wurden.

Es zeigte sich kein signifikanter Unterschied in Bezug auf die Häufigkeit von Akne. 30 PCOS-Patientinnen (29,41\%) und 16 Patientinnen ohne PCOS (27,59\%) litten an Akne, so dass ein p-Wert von 0,8060 ermittelt wurde.

\subsubsection{Hirsutismus}

In der Gruppe der Patientinnen ohne PCOS ergab sich eine signifikant unterschiedliche Häufigkeit von Hirsutismus unter Einnahme eines Ovulationshemmers (mit Ovulationshemmer: 18,60 \%; ohne Ovulationshemmer: 46,67 \%; $p=0,0326)$. Um eine bessere Aussage treffen zu können, wurden daher für den Parameter Hirsutismus in beiden Gruppen lediglich Patientinnen ohne Ovulationshemmer ausgewertet. 
Der Vergleich von 35 PCOS-Patientinnen (48,61\%) mit acht Patientinnen aus Gruppe 5 (18,60\%), die jeweils an Hirsutismus litten, ergab einen signifikanten Unterschied zwischen beiden Gruppen für diesen Parameter ( $p=0,0013)$.

\subsubsection{Zyklusstörungen}

Zyklusstörungen traten trotz Einnahme eines Ovulationshemmers noch bei mehr als der Hälfte der PCOS-Patientinnen auf ( $n=16 / 29: 55,17 \%)$. Es litten dennoch signifikant mehr PCOS-Patientinnen ohne Ovulationshemmer an Zyklusstörungen ( $n=60 / 72: 83,33 \%)$; ein $p-W e r t$ von 0,003 wurde hierfür errechnet.

Nach Ausschluss von Patientinnen mit Ovulationshemmern ergab sich ein hochsignifikant unterschiedliches Auftreten von Zyklusstörungen in beiden Gruppen $(p<0,00001)$. Wie gezeigt, hatten durchschnittlich vier von fünf PCOS-Patientinnen per Definition keinen normalen Zyklus, wohingegen dies nur bei knapp einem Drittel aller Frauen mit im Verlauf ausgeschlossenem PCOS zutraf ( $n=14 / 43: 32,56 \%)$.

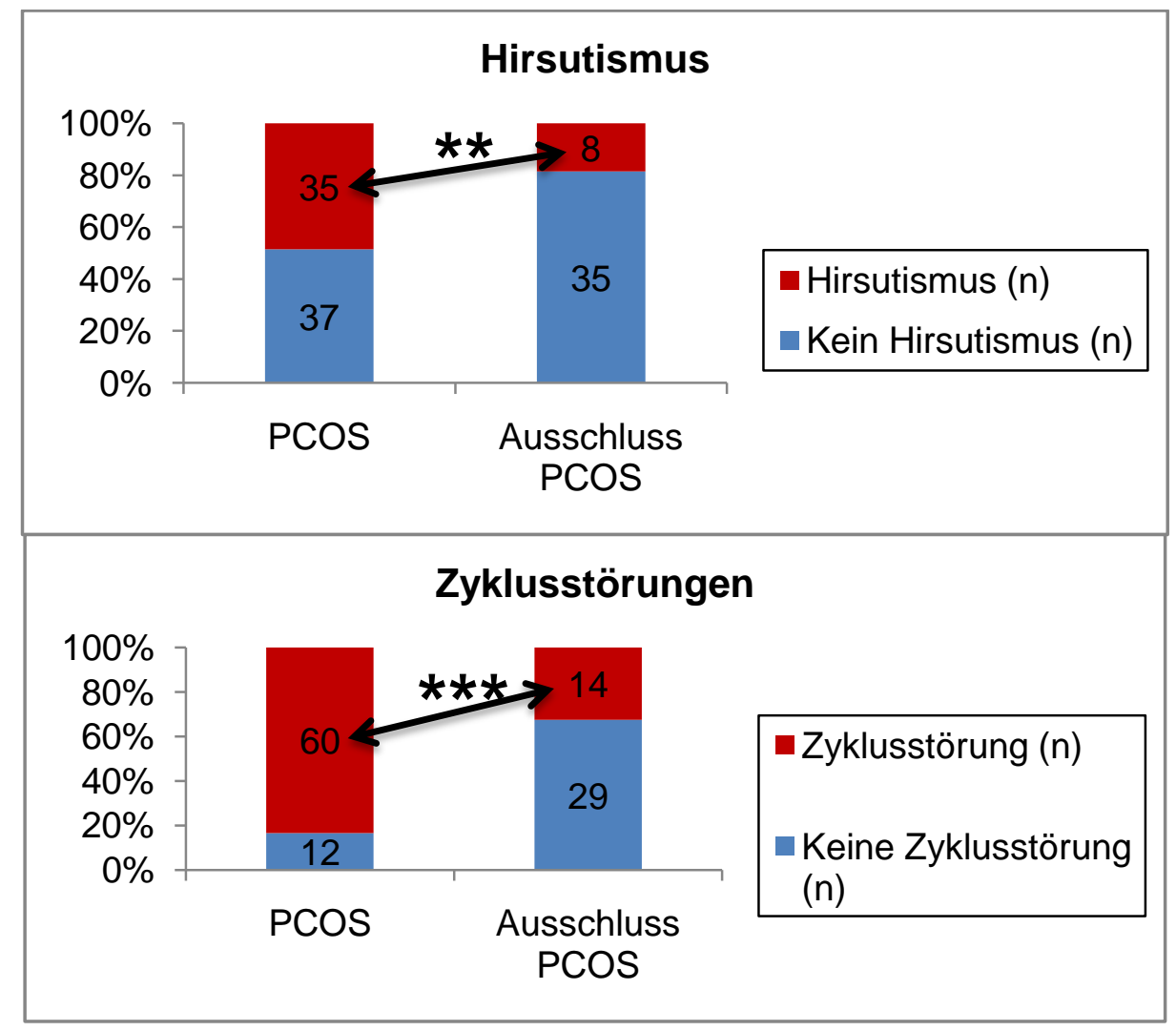

Abbildungen 12 und 13: Verteilung von Hirsutismus und Zyklusstörungen in beiden Gruppen. PCOS-Patientinnen wiesen die für das PCOS typischen Zeichen einer Hyperandrogenämie auf. Die Y-Achse gibt den prozentualen Anteil der Patientinnen an; die Zahl in der Säule die Anzahl der Patientinnen. 


\subsubsection{Besondere Nebendiagnosen und Medikation}

Die Nebendiagnosen und Vormedikation aller Patientinnen wurden zwar standardmäßig im Endokrinologikum erfasst, jedoch auf Grund der Vielzahl möglicher Erkrankungen und Medikamente nicht in die Auswertung dieser Arbeit mit eingebracht. Von Interesse waren hierbei lediglich die Diagnose einer Autoimmunthyreoiditis vom Typ Hashimoto und die vorbestehende Einnahme von Ovulationshemmern.

\subsubsection{Hashimoto-Thyreoiditis (AIT)}

Bei vierzehn PCOS-Patientinnen (13,73 \%) lag die Diagnose einer AIT bereits vor oder wurde im Verlauf der Vorstellung im Endokrinologikum gestellt. Es konnte hierbei kein signifikanter Unterschied zur Vergleichsgruppe 5 mit elf AIT-Patientinnen $(18,97 \%)$ festgestellt werden (Pearson-Chi-Quadrat-Test: $p=0,3802)$.

\subsubsection{Vormedikation mit Ovulationshemmern}

Obwohl beinah doppelt so viele PCOS-Patientinnen (29 vs. 15) einen Ovulationshemmer als Vormedikation hatten, war der Unterschied in relativer Häufigkeit mit $28,71 \%$ zu 25,86\% nicht signifikant (Pearson Chi-Quadrat-Test: $\mathrm{p}=0,6989$ ). Für die weiteren Auswertungen wurde der Einfluss von Ovulationshemmern jedoch bei ausgewählten Parametern einzeln betrachtet (s.o. und s.u.).

\subsubsection{Ergebnisse des OGTT}

Die Darstellung aller Ergebnisse des OGTT erfolgt in zwei Schritten.

Zunächst wurden für die Parameter Glukose, Insulin und C-Peptid jeweils die Werte nüchtern, nach 60 und nach 120 Minuten erhoben. Die aus diesen Werten errechneten Parameter AUC Glukose/Insulin/C-Peptid sowie die Scores zur Insulinresistenz HOMA-IR und QUICKI werden im Anschluss daran präsentiert. Als Test wurde ebenfalls der zweiseitige t-Test für unabhängige Stichproben gewählt. 


\subsubsection{Glukose, Insulin und C-Peptid}

Zwischen beiden Gruppen bestand kein signifikanter Unterschied in Bezug auf alle erhobenen Glukose-Werte. Mit Ausnahme des C-Peptid-Wertes nach 120 Minuten bestand jedoch bei sämtlichen anderen gemessenen Werten in beiden Gruppen ein signifikanter Unterschied.

\begin{tabular}{|c|c|c|c|c|c|c|c|c|}
\hline \multirow[b]{2}{*}{ Parameter } & \multirow[b]{2}{*}{ Norm } & \multicolumn{3}{|l|}{ Pcos } & \multicolumn{4}{|c|}{$\begin{array}{l}\text { Ausschluss } \\
\text { PCOS }\end{array}$} \\
\hline & & $\begin{array}{c}\text { Anzahl } \\
(\mathrm{n})\end{array}$ & $\begin{array}{l}\text { MW } \\
\pm \text { SD }\end{array}$ & Median & $\begin{array}{c}\text { Anzahl } \\
\text { (n) }\end{array}$ & $\begin{array}{l}\text { MW } \\
\pm \text { SD }\end{array}$ & Median & p-Wert \\
\hline $\begin{array}{c}\text { Glukose } \\
\text { nüchtern } \\
\text { (mg/dl) }\end{array}$ & $\begin{array}{l}N G T<90 \\
I G T<110 \\
D M \geq 200\end{array}$ & 102 & $\begin{array}{r}84,74 \\
\pm 13,10\end{array}$ & 84,00 & 58 & $\begin{array}{r}88,07 \\
\pm 9,82\end{array}$ & 85,00 & 0,0703 \\
\hline $\begin{array}{c}\text { Glukose } 1 \mathrm{~h} \\
\text { (mg/dl) }\end{array}$ & - & 102 & $\begin{array}{r}128,32 \\
\pm 36,13\end{array}$ & 122,00 & 58 & $\begin{array}{c}122,81 \\
\pm 39,48\end{array}$ & 111,50 & 0,3834 \\
\hline $\begin{array}{c}\text { Glukose } 2 \mathrm{~h} \\
\text { (mg/dl) }\end{array}$ & $\begin{array}{c}N G T<140 \\
\text { IGT:140-199 } \\
D M \geq 200\end{array}$ & 102 & $\begin{array}{r}100,62 \\
\pm 29,65\end{array}$ & 98,00 & 58 & $\begin{array}{c}95,41 \\
\pm 25,37\end{array}$ & 93,00 & 0,2433 \\
\hline $\begin{array}{c}\text { Insulin } \\
\text { nüchtern } \\
(\mu \mathrm{U} / \mathrm{ml})\end{array}$ & $<7$ & 102 & $\begin{array}{r}17,40 \\
\pm 17,51\end{array}$ & 12,30 & 58 & $\begin{array}{c}11,23 \\
\pm 16,27\end{array}$ & 8,65 & 0,0266 \\
\hline $\begin{array}{l}\text { Insulin } 1 \mathrm{~h} \\
(\mu \mathrm{U} / \mathrm{ml})\end{array}$ & - & 102 & $\begin{array}{c}132,57 \\
\pm \\
101,81\end{array}$ & 105,50 & 58 & $\begin{array}{c}83,20 \\
\pm 63,74\end{array}$ & 70,35 & 0,0002 \\
\hline $\begin{array}{l}\text { Insulin } 2 \text { h } \\
(\mu \mathrm{U} / \mathrm{ml})\end{array}$ & - & 102 & $\begin{array}{c}106,57 \\
\pm \\
104,40\end{array}$ & 70,55 & 58 & $\begin{array}{c}68,82 \\
\pm 68,47\end{array}$ & 54,75 & 0,0066 \\
\hline $\begin{array}{c}\text { C-Peptid } \\
\text { nüchtern } \\
\text { (ng/ml) }\end{array}$ & $0,9-4,0$ & 102 & $\begin{array}{c}2,71 \\
\pm 1,26\end{array}$ & 2,46 & 58 & $\begin{array}{r}2,29 \\
\pm 1,20\end{array}$ & 2,08 & 0,0370 \\
\hline $\begin{array}{c}\text { C-Peptid 1h } \\
\text { (ng/ml) }\end{array}$ & - & 102 & $\begin{array}{c}9,82 \\
\pm 3,52\end{array}$ & 9,33 & 58 & $\begin{array}{c}8,71 \\
\pm 2,46\end{array}$ & 8,77 & 0,0204 \\
\hline $\begin{array}{l}\text { C-Peptid } 2 \mathrm{~h} \\
\text { (ng/ml) }\end{array}$ & - & 102 & $\begin{array}{c}9,12 \\
\pm 3,76\end{array}$ & 8,58 & 58 & $\begin{array}{c}8,49 \\
\pm 3,11\end{array}$ & 8,15 & 0,2533 \\
\hline
\end{tabular}

Tabelle 9: Ergebnisse des OGTT. Für Insulin und C-Peptid existieren diesbezüglich noch keine Grenzwerte. In beiden Patientengruppen bestand per Definition keine pathologische (=gestörte) Glukosetoleranz. (Abkürzungen: NGT=Normale Glukosetoleranz; IGT= Gestörte Glukosetoleranz; $\mathrm{DM}=$ Diabetes mellitus) 


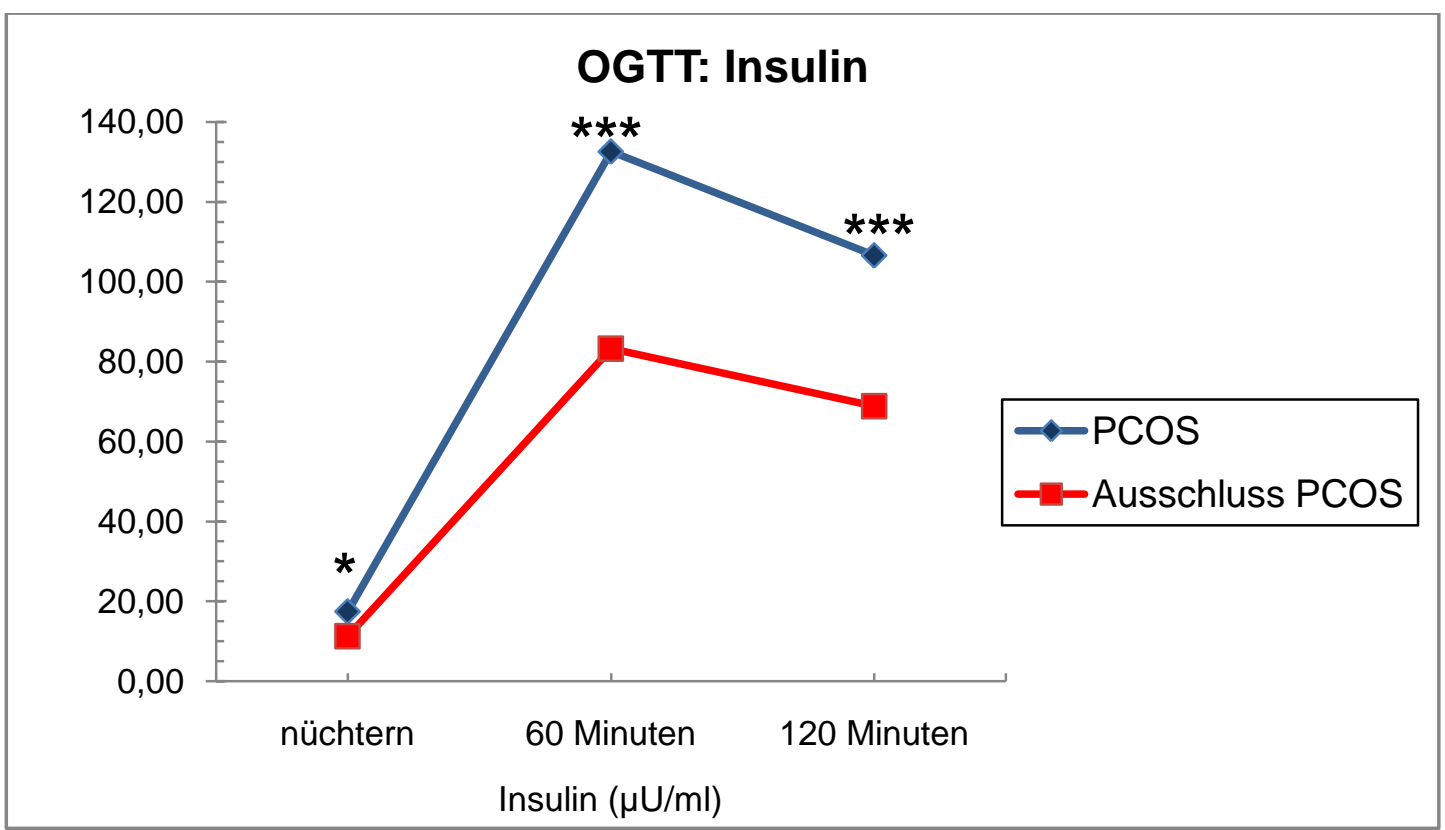

Abbildung 14: Insulin-Mittelwerte im OGTT. PCOS-Patientinnen hatten signifikant höhere InsulinNüchternwerte sowie signifikant höhere Insulin-Werte nach 60 und 120 Minuten. [Derzeit existieren keine Standards zur klinischen Einschätzung der Hyperinsulinämie im OGTT; als Richtwerte für eine Hyperinsulinämie nach ein bis zwei Stunden im OGTT geben Azziz et al. (2009) Werte ab $\underline{\mathbf{8 0 - 1 0 0}}$ $\underline{\mu \mathrm{U} / \mathrm{ml}}$ an, eine schwere Hyperinsulinämie liege bei Werten $\geqq \mathbf{3 0 0} \mu \mathrm{U} / \mathrm{ml}$ vor.]

\subsubsection{AUC Glukose, AUC Insulin, AUC C-Peptid}

Die errechnete Fläche unter der Kurve (AUC) war allein für den Parameter Insulin signifikant unterschiedlich in beiden Gruppen.

\begin{tabular}{|c|c|c|c|c|c|c|c|}
\hline \multirow[b]{2}{*}{ Parameter } & \multicolumn{3}{|l|}{ PCOS } & \multicolumn{3}{|c|}{ Ausschluss PCOS } & \multirow[b]{2}{*}{ p-Wert } \\
\hline & $\begin{array}{c}\text { Anzahl } \\
\text { (n) }\end{array}$ & $\begin{array}{l}\text { MW } \\
\pm \text { SD }\end{array}$ & Median & $\begin{array}{l}\text { Anzahl } \\
\text { (n) }\end{array}$ & $\begin{array}{l}\text { MW } \\
\pm \text { SD }\end{array}$ & Median & \\
\hline $\begin{array}{l}\text { AUC Glukose } \\
\text { (mg/dl/min) }\end{array}$ & 102 & $\begin{array}{r}162,03 \\
\pm 34,30\end{array}$ & 162,00 & 58 & $\begin{array}{r}155,26 \\
\pm 36,34\end{array}$ & 150,00 & 0,2501 \\
\hline $\begin{array}{l}\text { AUC Insulin } \\
(\mu \mathrm{U} / \mathrm{ml} / \mathrm{min})\end{array}$ & 102 & $\begin{array}{r}150,92 \\
\pm 124,00\end{array}$ & 113,50 & 58 & $\begin{array}{r}95,98 \\
\pm 82,19\end{array}$ & 76,50 & 0,0010 \\
\hline $\begin{array}{c}\text { AUC } \\
\text { C-Peptid } \\
(\mathrm{ng} / \mathrm{ml} / \mathrm{min})\end{array}$ & 102 & $\begin{array}{r}12,47 \\
\pm 4,35\end{array}$ & 12,00 & 58 & $\begin{array}{r}11,28 \\
\pm 3,51\end{array}$ & 11,00 & 0,0604 \\
\hline
\end{tabular}

Tabelle 10: Fläche unter der Kurve von Glukose, Insulin und C-Peptid. 


\subsubsection{HOMA-IR und QUICKI}

Für beide errechneten Scores ergaben sich Signifikanzen.

Der Mittelwert des HOMA-IR lag bei PCOS-Patientinnen mit 3,84 ( \pm 4,76 SD; Median: 2,70) im Bereich einer als wahrscheinlich anzunehmenden Insulinresistenz, wohingegen Patientinnen ohne PCOS mit einem Mittelwert von 2,48 $( \pm 3,78$ SD; Median: 1,75) knapp unter dem Cut-Off-Wert von 2,5 blieben. Dies entspricht einem p-Wert von 0,0488. Unter Berücksichtigung des Einflusses von Ovulationshemmern (siehe Abschnitt 3.3.6) hatten die PCOS-Patientinnen ohne Ovulationshemmer einen noch höheren HOMA-IR von durchschnittlich 4,28. Bei Patientinnen ohne PCOS war der Einfluss des Ovulationshemmers auf den HOMA-IR nicht signifikant.

Hochsignifikant $(p=0,0025)$ gestalteten sich die Ergebnisse des QUICKI. Patientinnen mit PCOS erzielten niedrigere Werte (MW: 0,33 \pm 0,04 SD; Median: 0,33) als Patientinnen der Gruppe 5 (MW: 0,35 \pm 0,04 SD; Median: 0,35). Zum besseren Verständnis und in Abgrenzung zum HOMA-IR gilt folgende Anmerkung: Je niedriger der Wert des QUICKI (=niedrigere Insulinsensitivität), desto größer die Ausprägung einer Insulinresistenz - 0,45 bei gesunden Menschen bis 0,30 bei Diabetikern.

\subsubsection{Einfluss von Ovulationshemmern}

Da bei 29 PCOS-Patientinnen (28,71\%) und bei 15 Patientinnen ohne PCOS $(25,86$ \%) die Vormedikation zum Untersuchungszeitpunkt einen Ovulationshemmer enthielt, stellte sich die Frage nach einem potenziellen Einfluss des Ovulationshemmers auf bestimmte Parameter. Es wurden einerseits der LH/FSHQuotient, der freie Androgenindex und das SHBG, andererseits der HOMA-IR und die Fläche unter der Kurve von Insulin als Parameter ausgewählt. Die pharmakologische Wirkungsweise des Ovulationshemmers - Ein-/MehrphasenPräparat oder sog. Minipille oder antiandrogener Ovulationshemmer - wurde hierbei nicht differenziert.

\subsubsection{Ovulationshemmer bei PCOS-Patientinnen}

Unter Einnahme eines Ovulationshemmers sanken signifikant die Werte des HOMAIR und des FAI. Das SHBG stieg signifikant an. 


\begin{tabular}{|c|c|c|c|c|c|c|c|}
\hline \multirow[b]{2}{*}{ Parameter } & \multicolumn{3}{|c|}{ Ovulationshemmer } & \multicolumn{3}{|c|}{ Kein Ovulationshemmer } & \multirow[b]{2}{*}{ p-Wert } \\
\hline & $\begin{array}{c}\text { Anzahl } \\
\text { (n) }\end{array}$ & $\begin{array}{l}\text { MW } \\
\pm \text { SD }\end{array}$ & Median & $\begin{array}{c}\text { Anzahl } \\
\text { (n) }\end{array}$ & $\begin{array}{l}\text { MW } \\
\pm \text { SD }\end{array}$ & Median & \\
\hline $\begin{array}{l}\text { AUC Insulin } \\
(\mu \mathrm{U} / \mathrm{ml} / \mathrm{min})\end{array}$ & 29 & $\begin{array}{r}133,45 \\
\pm 72,60\end{array}$ & 118,00 & 72 & $\begin{array}{r}156,19 \\
\pm 139,34\end{array}$ & 109,50 & 0,2871 \\
\hline HOMA-IR & 29 & $\begin{array}{c}2,58 \\
\pm 1,49\end{array}$ & 2,10 & 72 & $\begin{array}{c}4,28 \\
\pm 5,49\end{array}$ & 2,75 & 0,0177 \\
\hline $\begin{array}{c}\text { BMI } \\
\left(\mathrm{kg} / \mathrm{m}^{2}\right)\end{array}$ & 29 & $\begin{array}{l}29,75 \\
\pm 8,51\end{array}$ & 30,42 & 72 & $\begin{array}{r}33,25 \\
\pm 6,80\end{array}$ & 34,35 & 0,0544 \\
\hline FAI & 29 & $\begin{array}{c}3,20 \\
\pm 5,19\end{array}$ & 1,00 & 70 & $\begin{array}{c}9,94 \\
\pm 6,98\end{array}$ & 7,95 & 0,000002 \\
\hline LH/FSH & 26 & $\begin{array}{c}1,50 \\
\pm 1,12\end{array}$ & 1,11 & 68 & $\begin{array}{c}1,69 \\
\pm 0,85\end{array}$ & 1,50 & 0,4348 \\
\hline $\begin{array}{c}\text { SHBG } \\
(\mathrm{nmol} / \mathrm{l})\end{array}$ & 29 & $\begin{array}{r}113,19 \\
\pm 59,05\end{array}$ & 123,00 & 71 & $\begin{array}{c}38,00 \\
\pm 33,51\end{array}$ & 31,70 & 0,000001 \\
\hline
\end{tabular}

Tabelle 11: Einfluss des Ovulationshemmers auf Parameter von PCOS-Patientinnen. Der Ovulationshemmer hatte im zweiseitigen $t$-Test für unabhängige Stichproben einen Einfluss auf den FAI, das SHBG und den HOMA-IR. Eine Tendenz bestand für den BMI.

\subsubsection{Ovulationshemmer bei Patientinnen ohne PCOS}

In der Vergleichsgruppe der Patientinnen mit ausgeschlossenem PCOS hatte der Ovulationshemmer einen Einfluss auf den FAI und das SHBG. Eine Tendenz bestand wie bei PCOS-Patientinnen für den BMI.

\begin{tabular}{|c|c|c|c|c|c|c|c|}
\hline \multirow[b]{2}{*}{ Parameter } & \multicolumn{3}{|c|}{ Ovulationshemmer } & \multicolumn{3}{|c|}{ Kein Ovulationshemmer } & \multirow[b]{2}{*}{ p-Wert } \\
\hline & $\begin{array}{c}\text { Anzahl } \\
(n)\end{array}$ & $\begin{array}{l}\text { MW } \\
\pm \text { SD }\end{array}$ & Median & $\begin{array}{c}\text { Anzahl } \\
\text { (n) }\end{array}$ & $\begin{array}{l}M W \\
\pm S D\end{array}$ & Median & \\
\hline $\begin{array}{l}\text { AUC Insulin } \\
(\mu \mathrm{U} / \mathrm{ml} / \mathrm{min})\end{array}$ & 15 & $\begin{array}{c}84,45 \\
\pm 29,45\end{array}$ & 77,00 & 43 & $\begin{array}{r}100,00 \\
\pm 93,88\end{array}$ & 76,00 & 0,3421 \\
\hline HOMA-IR & 15 & $\begin{array}{c}1,87 \\
\pm 0,83\end{array}$ & 1,80 & 43 & $\begin{array}{c}2,70 \\
\pm 4,36\end{array}$ & 1,70 & 0,2408 \\
\hline $\begin{array}{c}\text { BMI } \\
\left(\mathbf{k g} / \mathbf{m}^{2}\right)\end{array}$ & 15 & $\begin{array}{r}26,86 \\
\pm 5,83\end{array}$ & 28,60 & 43 & $\begin{array}{r}31,00 \\
\pm 9,43\end{array}$ & 29,70 & 0,0541 \\
\hline FAl & 14 & $\begin{array}{c}1,66 \\
\pm 2,17\end{array}$ & 0,80 & 43 & $\begin{array}{c}4,19 \\
\pm 4,46\end{array}$ & 2,60 & 0,0067 \\
\hline LH/FSH & 14 & $\begin{array}{r}1,20 \\
\pm 1,22\end{array}$ & 0,90 & 41 & $\begin{array}{r}1,30 \\
\pm 0,79\end{array}$ & 0,93 & 0,7765 \\
\hline $\begin{array}{l}\text { SHBG } \\
(\mathrm{nmol} / \mathrm{l})\end{array}$ & 14 & $\begin{array}{r}122,56 \\
\pm 67,48\end{array}$ & 162,75 & 42 & $\begin{array}{c}41,34 \\
\pm 17,71\end{array}$ & 36,50 & 0,0006 \\
\hline
\end{tabular}

Tabelle 12: Einfluss des Ovulationshemmers auf Parameter von Patientinnen ohne PCOS. 


\subsubsection{LH/FSH, SHBG und FAI}

Aus der Vielzahl an im Endokrinologikum erhobenen Laborparametern und HormonIndizes wurden drei für das PCOS interessante Indizes ausgewählt und mittels zweiseitigen t-Tests für unabhängige Stichproben ausgewertet. Dabei wurde der Einfluss von Ovulationshemmern berücksichtigt.

\subsubsection{LH/FSH}

Der Einfluss von Ovulationshemmern auf den LH/FSH-Quotienten war in beiden Gruppen nicht signifikant (siehe Abschnitt 3.3.6). Folglich wurde jeweils die gesamte Gruppe unabhängig von der Einnahme eines Ovulationshemmers ausgewertet.

PCOS-Patientinnen hatten mit 1,64 ( \pm 0,93 SD; Median: 1,45) im Durchschnitt einen signifikant höheren LH/FSH-Quotienten als Patientinnen ohne PCOS (MW: 1,28 \pm 0,91 SD; Median: 0,93), was einem p-Wert von 0,0210 entspricht. Dennoch lag der Quotient bei PCOS-Patientinnen noch unter dem für das PCOS typischen Wert von 2,0 .

Eine Korrelation zwischen dem BMI, welcher ebenfalls nicht signifikant von der Einnahme eines Ovulationshemmers beeinflusst war, und dem LH/FSH-Quotienten ergab in beiden Gruppen keinen statistischen Zusammenhang. Um eine differenziertere Aussage treffen zu können, wurden Subanalysen beider Gruppen mit Korrelationen zweier ausgewählter BMI-Grade (<25 vs. $\geq 25 \mathrm{~kg} / \mathrm{m}^{2}$ und $<30 \mathrm{vs}$. $\geq 30$ $\mathrm{kg} / \mathrm{m}^{2}$ ) durchgeführt. Bei adipösen PCOS-Patientinnen mit einem BMI $\geq 30 \mathrm{~kg} / \mathrm{m}^{2}$ $\left(r=0,2571 ; p=0,0473 ; r^{2}=0,0661\right)$ und bei normalgewichtigen Patientinnen ohne PCOS mit einem BMl $<25 \mathrm{~kg} / \mathrm{m}^{2}\left(r=-0,4926 ; p=0,0378 ; r^{2}=0,2426\right)$ hatte der BMI Einfluss auf die Höhe des LH/FSH-Quotienten. Sämtliche anderen Subanalysen erreichten keine statistische Signifikanz. 


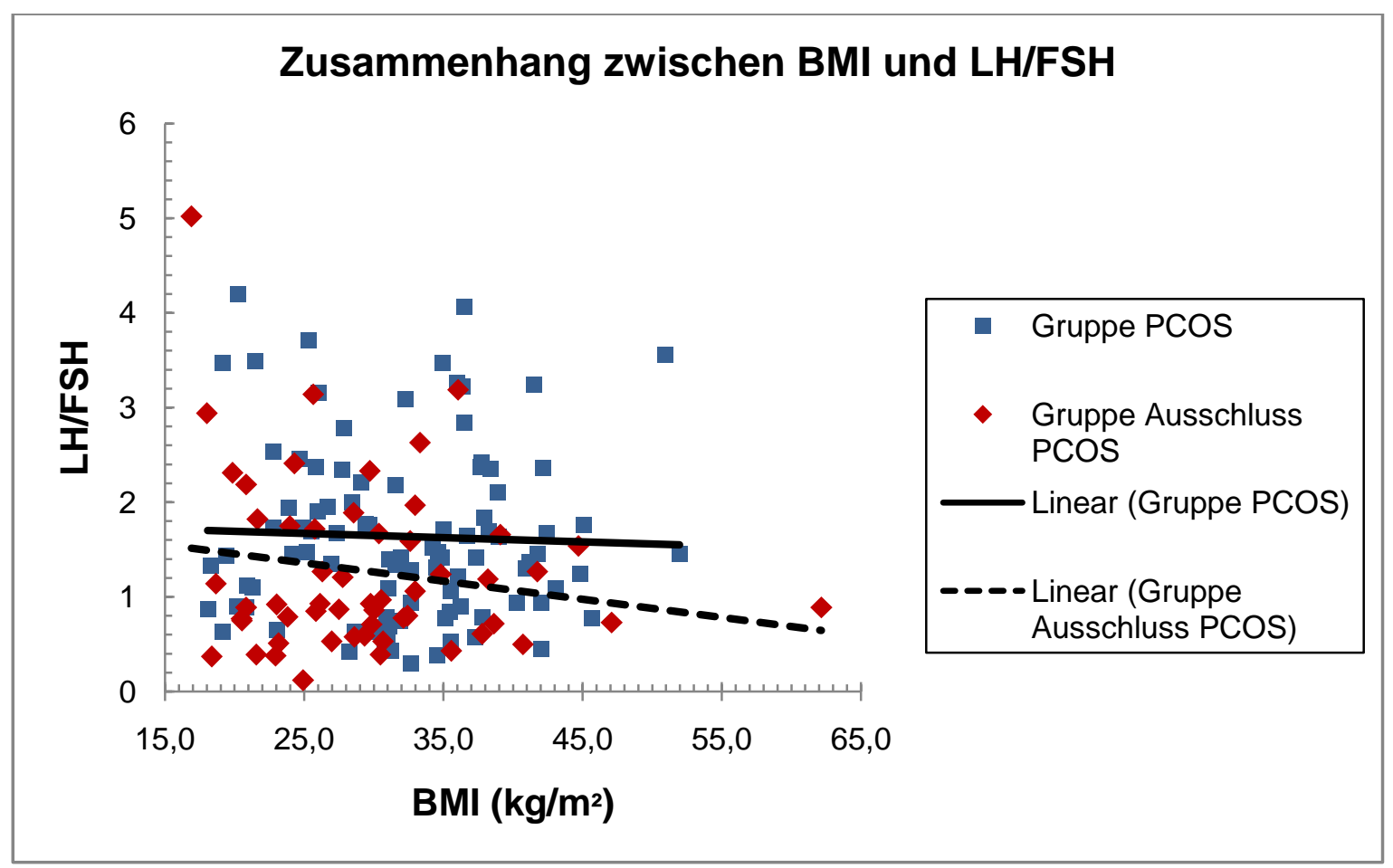

Abbildung 15: Zusammenhang zwischen BMI und LH/FSH-Quotient in beiden Gruppen. (Gruppe PCOS: $r=-0,0362 ; p=0,7274 ; r^{2}=0,0013$. Gruppe Ausschluss PCOS: $r=-0,1762 ; p=0,1982 ; r^{2}=0,0310$ ) Der BMI bedingte in beiden Gruppen nicht die Höhe des LH/FSH-Quotienten.

Eine Korrelation zwischen dem Alter der Patientinnen und dem LH/FSH-Quotienten ergab bei PCOS-Patientinnen einen tendenziellen ( $\left.r=-0,1900 ; p=0,0651 ; r^{2}=0,0361\right)$ und bei Patientinnen ohne PCOS einen signifikanten statistischen Zusammenhang $\left(r=-0,3942 ; p=0,0029 ; r^{2}=0,1554\right)$.

\subsubsection{SHBG}

Unter der Einnahme eines Ovulationshemmers hatten Patientinnen in beiden Gruppen signifikant höhere SHBG-Werte, so dass bei Auswertung dieses Parameters nur die Patientinnen ohne Ovulationshemmer berücksichtig wurden.

Das SHBG war bei PCOS-Patientinnen mit durchschnittlich 38,00 nmol/l $( \pm 33,51$ nmol/l SD; Median: $31,70 \mathrm{nmol} / \mathrm{l})$ nicht signifikant niedriger als bei Patientinnen ohne PCOS mit durchschnittlich 41,72 nmol/l ( $\pm 17,67 \mathrm{nmol} / \mathrm{l} \mathrm{SD;}$; Median: 36,95 nmol/l), was einem p-Wert von 0,4420 entspricht. Beide Mittelwerte liegen im Referenzbereich für Frauen (18,0-144,0 nmol/l). 
Wie bereits in Abschnitt 3.3.2 dargestellt, waren PCOS-Patientinnen knapp sieben Jahre jünger. Auch nach Ausschluss aller Patientinnen mit Ovulationshemmer verblieb ein signifikanter Altersunterschied von etwa 7,5 Jahren zwischen beiden Gruppen $(p=0,0010)$.

Korrelationen zwischen der Fläche unter der Kurve von Insulin (AUC Insulin) mit dem SHBG ergaben bei PCOS-Patientinnen keinen statistisch signifikanten Zusammenhang, wohl aber bei Patientinnen ohne PCOS.

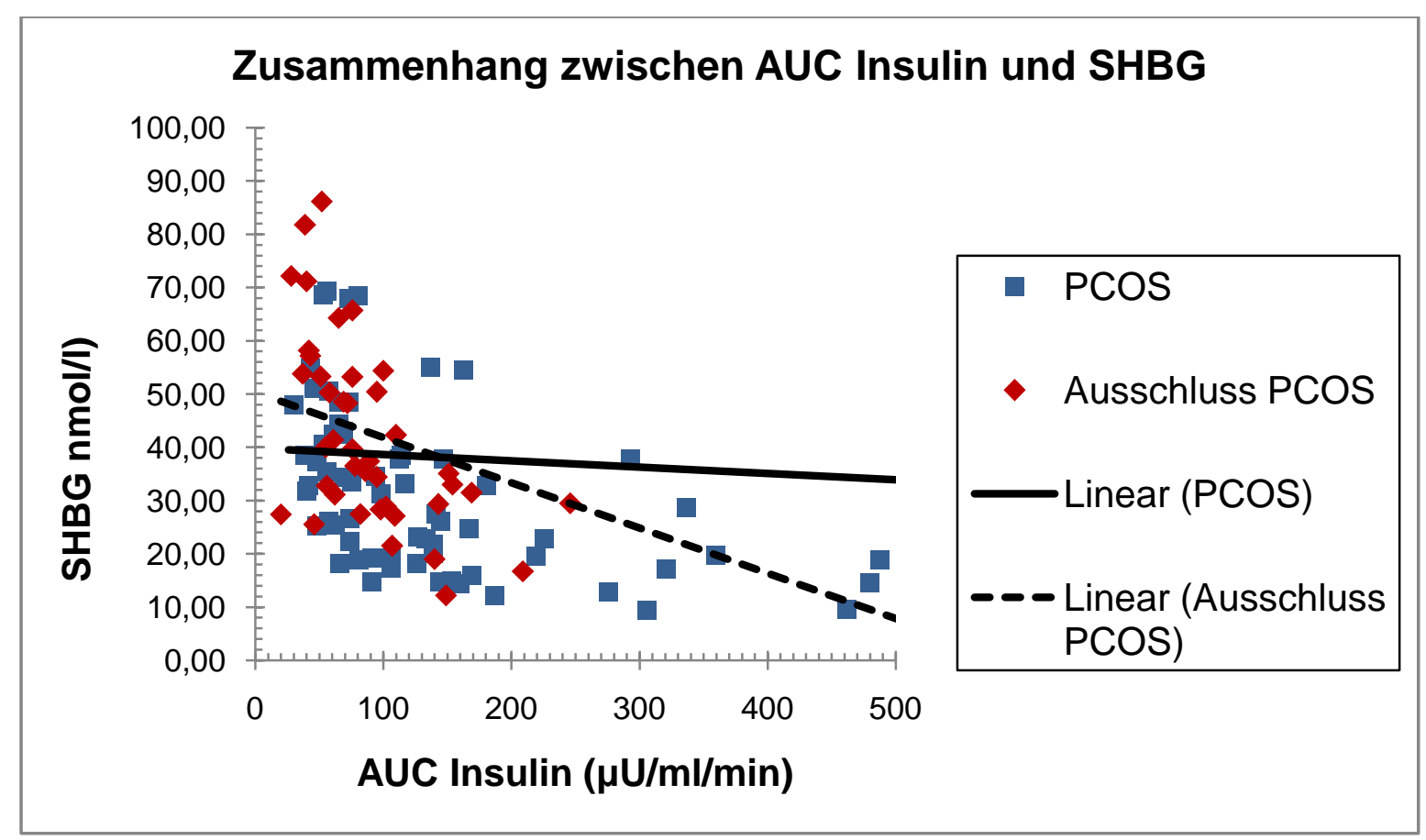

Abbildung 16: Zusammenhang von AUC Insulin und SHBG in beiden Gruppen. (Gruppe PCOS: $r=-0,0486 ; p=0,6873 ; r^{2}=0,0024$. Gruppe Ausschluss PCOS: $\left.r=-0,4557 ; p=0,0024 ; r^{2}=0,2077\right)$.

Bei Patientinnen ohne PCOS sinken die SHBG-Spiegel bei erhöhten Insulinwerten im Blut.

Der HOMA-IR war in beiden Gruppen nicht mit dem SHBG korreliert (PCOS: $r=$ -0,0692; $\mathrm{p}=0,5663 ; \mathrm{r}^{2}=0,0048$. Ausschluss PCOS: $\left.\mathrm{r}=-0,2449 ; \mathrm{p}=0,1180 ; \mathrm{r}^{2}=0,0600\right)$. Auch das Nüchterninsulin war jeweils nicht mit dem SHBG korreliert (PCOS: $r=-$ 0,0375; $\mathrm{p}=0,7565 ; \mathrm{r}^{2}=0,0014$. Ausschluss PCOS: $\mathrm{r}=-0,2492 ; \mathrm{p}=0,1115 ; \mathrm{r}^{2}=0,0621$ ). Dagegen lag bei Patientinnen ohne PCOS eine statistisch signifikante Korrelation von SHBG mit dem BMI vor $\left(r=-0,4820 ; p=0,0012 ; r^{2}=0,2324\right)$, bei PCOSPatientinnen lediglich in der Tendenz $\left(r=-0,2288 ; p=0,0550 ; r^{2}=0,0523\right)$. Zusammenfassend wurde das SHBG bei PCOS-Patientinnen in dieser Studie nicht vom HOMA-IR und der Höhe des Insulins bedingt. 


\subsubsection{FAl}

Wie auch beim SHBG war der Einfluss von Ovulationshemmern auf den freien Androgenindex in beiden Gruppen statistisch bedeutend, da in beiden Gruppen die Patientinnen mit Ovulationshemmer einen signifikant niedrigeren FAI hatten. Aus diesem Grund wurden wiederum nur Patientinnen ohne Ovulationshemmer für die Auswertung des FAl berücksichtigt.

PCOS-Patientinnen hatten im Vergleich zu Patientinnen ohne PCOS einen mehr als doppelt so hohen FAI-Wert (PCOS MW: $9.94 \pm$ 6,98. Siehe Abschnitt 3.3.6), was einem p-Wert von 0,0000004 entspricht. Die Mittelwerte beider Gruppen lagen über dem für Frauen gültigen Cut-Off-Wert von 3,5. Allerdings war der Anteil der Patientinnen mit einem FAI $\geq 3,5$ in der PCOS-Gruppe deutlich höher $(87,14 \%$ gegenüber $46,51 \%)$.

Eine Korrelation zwischen der Fläche unter der Kurve (AUC) von Insulin und dem FAl ergab für beide Gruppen einen hochsignifikanten statistischen Zusammenhang.

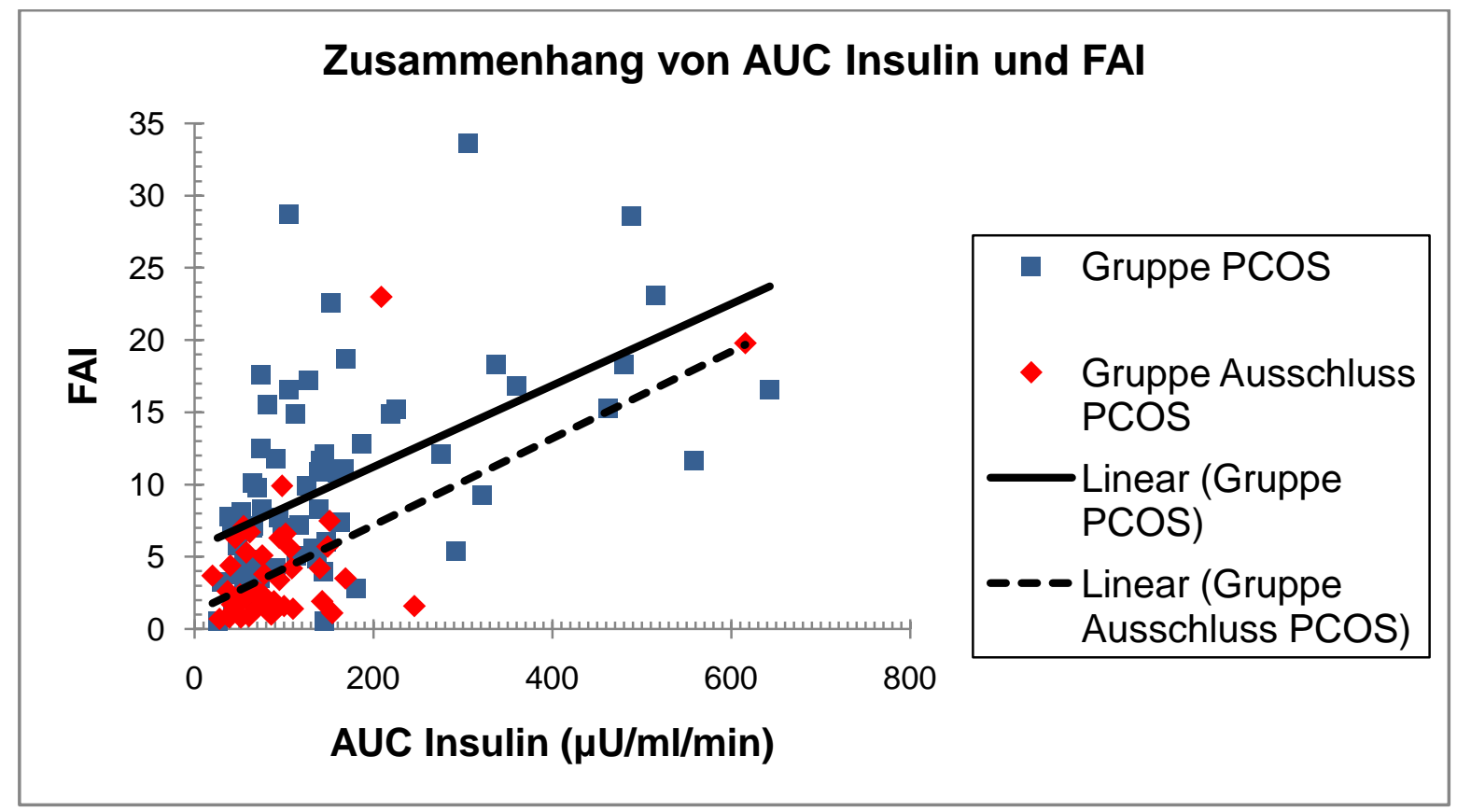

Abbildung 17: Zusammenhang zwischen AUC Insulin und FAI in beiden Gruppen. (Gruppe PCOS: $r=0,5581 ; p=0,000001 ; r^{2}=0,3115$. Gruppe Ausschluss PCOS: $r=0,6330 ; p=00001, r^{2}=0,4006$ ) In beiden Gruppen bedingt eine hohe Insulinausschüttung ein hohen FAI. 
Selbst bei Einschluss der Patientinnen, die einen Ovulationshemmer einnahmen, blieb die Korrelation statistisch hochsignifikant (PCOS: $r=0,4437 ; p=0,000001$; $r^{2}=0,1968$. Ausschluss PCOS: $\left.r=0,6222 ; p=0,000001 ; r^{2}=0,3871\right)$.

Im Gegensatz zur AUC Insulin war der Zusammenhang zwischen dem HOMA-IR und dem FAI lediglich bei PCOS-Patientinnen statistisch signifikant, wenngleich auch das Bestimmtheitsmaß $r^{2}$ sehr klein ausfiel.

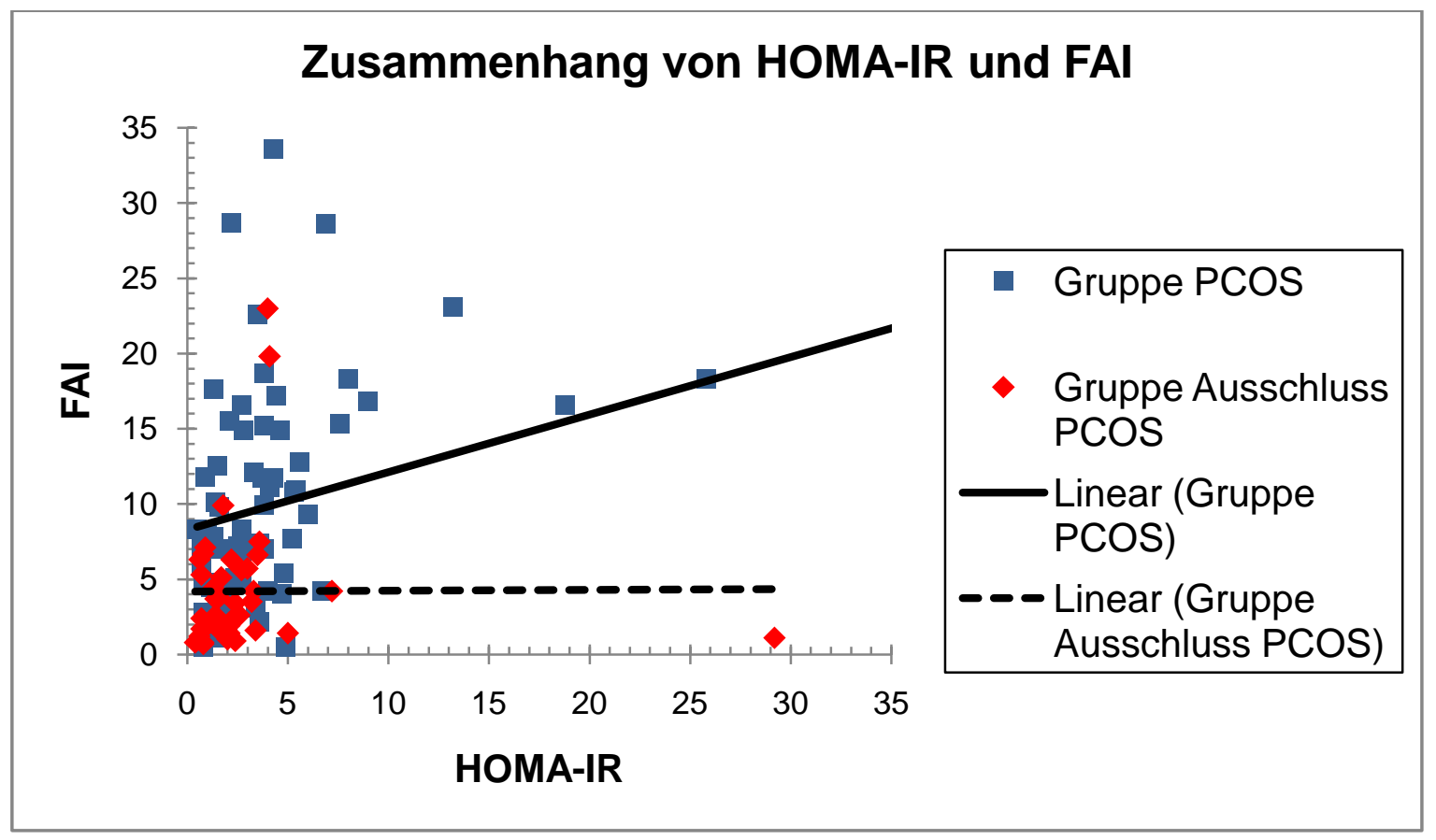

Abbildung 18: Zusammenhang zwischen HOMA-IR und FAl in beiden Gruppen. (Gruppe PCOS: $r=0,3037 ; p=0,0106 ; r^{2}=0,0922$. Gruppe Ausschluss PCOS: $\left.r=0,0057 ; p=0,9712 ; r^{2}=0,00001\right)$ Der HOMA-IR (als Indikator einer Insulinresistenz) führt bei PCOS-Patientinnen zu einem hohen FAI. Dagegen ist bei Patientinnen ohne PCOS der HOMA-IR nicht sensitiv genug für den FAI.

\subsection{Therapie der Insulinresistenz bei PCOS-Patientinnen mit Metformin}

Aus dem eingangs beschriebenen Gesamtkollektiv bietet die Gruppe PCOS-Met (1) den Vergleich zweier Untersuchungszeitpunkte und folglich die Beurteilung der Metformintherapie bei PCOS-Patientinnen mit Insulinresistenz. Diese Gruppe besteht ausschließlich aus Patientinnen mit gesichertem PCOS, was fortan - um Redundanzen zu vermeiden - nicht mehr explizit erwähnt wird.

Analog zu Abschnitt 3.3 werden die Ergebnisse in sinnvollen Abschnitten zusammengefasst und dargestellt. Als Test für metrische Parameter wurde der t-Test für 
gepaarte Stichproben gewählt. Alle nicht-parametrischen Daten wurden mit dem Wilcoxon-Test für gepaarte Stichproben ausgewertet.

\subsubsection{Klinische Basisparameter}

\subsubsection{Alter}

Die Patientinnen waren zum Zeitpunkt der zweiten Untersuchung zwischen 16 und 49 Jahre alt. Sie waren im Mittel knapp ein Jahr älter als bei ihrer Erstuntersuchung (MW: 28,30 \pm 8,83 SD), der Median lag bei 27 Jahren.

\subsubsection{Gewicht und BMI}

Gewicht und BMI waren zu beiden Untersuchungszeitpunkten nicht von der Einnahme eines Ovulationshemmers beeinflusst.

Sowohl die Spannweite des Gewichts (60 bis $120 \mathrm{~kg}$ ), als auch die des BMI (22 bis $44 \mathrm{~kg} / \mathrm{m}^{2}$ ) waren bei beiden Untersuchungen groß, so dass eine heterogene Patientengruppe betrachtet wurde.

\begin{tabular}{|c|c|c|c|c|c|c|c|}
\hline \multirow[b]{2}{*}{ Parameter } & \multicolumn{3}{|c|}{ Erstuntersuchung } & \multicolumn{3}{|c|}{ Zweituntersuchung } & \multirow[b]{2}{*}{ p-Wert } \\
\hline & $\begin{array}{l}\text { Anzahl } \\
\text { (n) }\end{array}$ & $\begin{array}{l}\text { MW } \\
\pm S D\end{array}$ & Median & $\begin{array}{l}\text { Anzahl } \\
\text { (n) }\end{array}$ & $\begin{array}{l}\text { MW } \\
\pm \text { SD }\end{array}$ & Median & \\
\hline Gewicht (kg) & 30 & $\begin{array}{r}91,62 \\
\pm 15,27\end{array}$ & 89,55 & 30 & $\begin{array}{r}87,54 \\
\pm 15,92\end{array}$ & 83,25 & 0,0009 \\
\hline BMI $\left(\mathbf{k g} / \mathbf{m}^{2}\right)$ & 30 & $\begin{array}{l}33,70 \\
\pm 5,13\end{array}$ & 34,39 & 30 & $\begin{array}{l}32,16 \\
\pm 5,23\end{array}$ & 31,22 & 0,0007 \\
\hline
\end{tabular}

Tabelle 13: Gewicht und BMI zu beiden Untersuchungszeitpunkten.

Im Durchschnitt nahmen alle Patientinnen vier Kilogramm an Körpergewicht ab, obwohl auch bei gut einem Viertel der Patientinnen eine Gewichtszunahme von 0,1 bis $8,0 \mathrm{~kg}$ zu verzeichnen war. Die Spannweite der Gewichtsabnahme erstreckte sich von 0,2 bis $21,7 \mathrm{~kg}$.

Die signifikante Gewichtsabnahme spiegelte sich auch im BMI wider, welcher um durchschnittlich 1,5 Punkte sank. Auch die Abnahme des BMl erstreckte sich auf eine Spannweite von 0,1 bis 7,8 Punkten. 


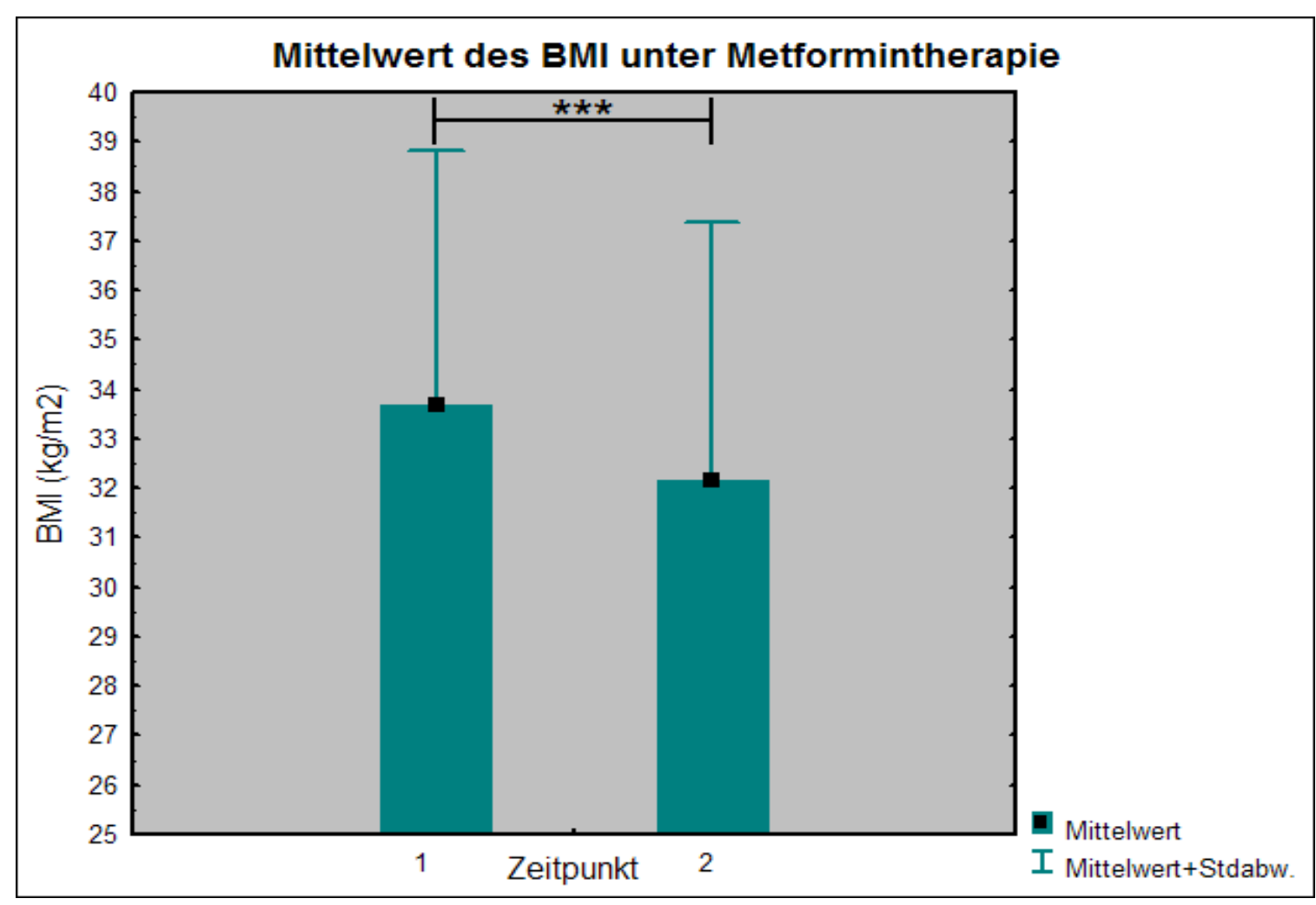

Tabelle 14: BMI unter der Therapie mit Metformin. Die Abnahme um durchschnittlich 1,5 Punkte ist hochsignifikant $(p=0,0007)$.

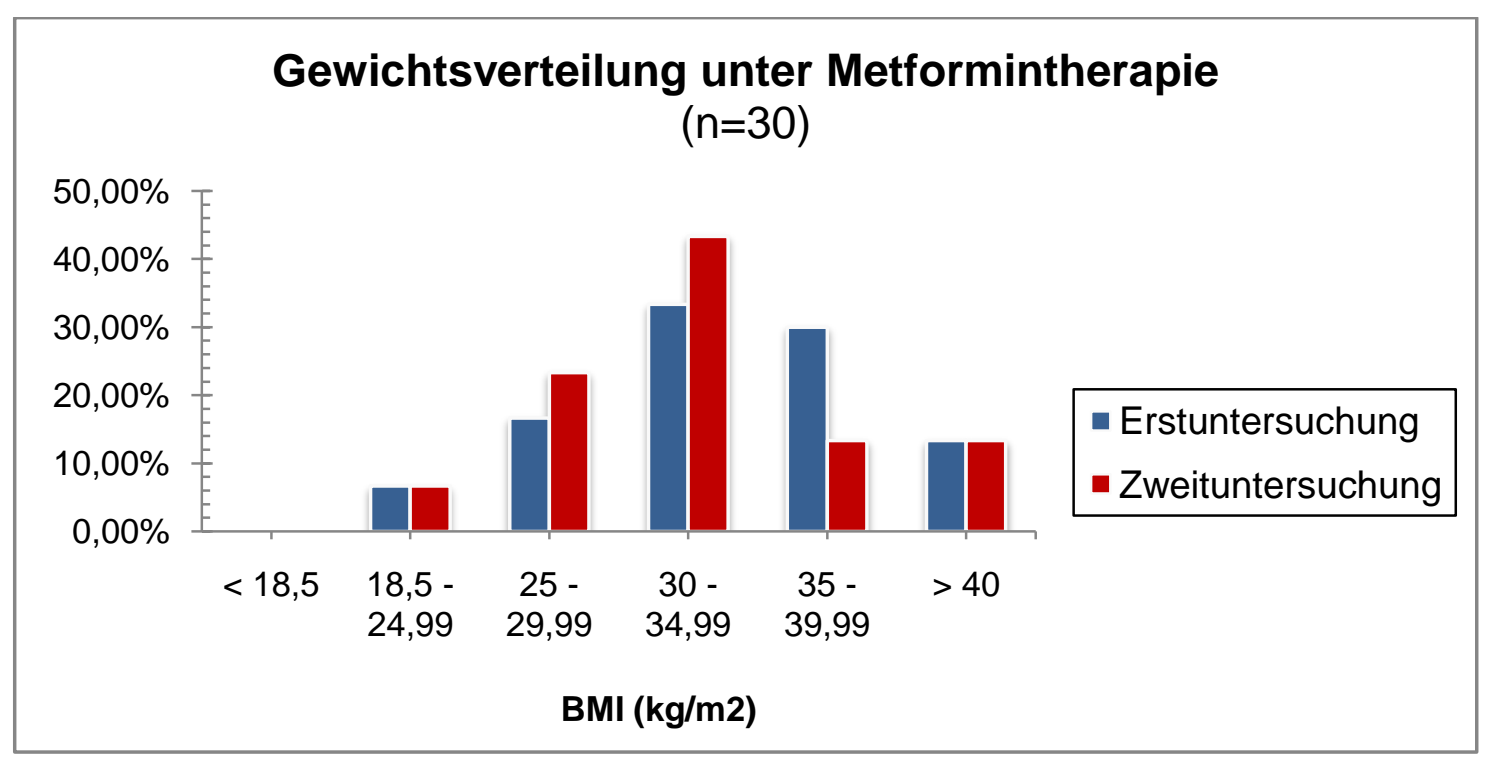

Abbildung 19: Gewichtsverteilung zu beiden Untersuchungszeitpunkten. Fünf Patientinnen hatten nicht mehr den Adipositas Grad II. (BMI in $\mathrm{kg} / \mathrm{m}^{2}$ : Untergewicht: $B M \mathrm{~K}<18,5$. Normalgewicht: $B M I=18,5-24,99$. Übergewicht/Präadipositas: $B M I=25-29,99$. Adipositas Grad I: $B M I=30-34,99$. Adipositas Grad II: BMI=35-39,99. Adipositas Grad III: BMI $\geq 40$ ) 


\subsubsection{Klinische PCOS-Parameter}

Ovulationshemmer hatten einen statistisch relevanten Einfluss auf die Parameter Akne, Hirsutismus und Zyklusstörungen. Um validere Aussagen zur Therapie mit Metformin treffen zu können, wurden alle Patientinnen mit Ovulationshemmer von der Auswertung ausgeschlossen. Es verblieben 19 Patientinnen.

\subsubsection{Akne}

Es konnte kein signifikanter Rückgang des Auftretens von Akne zum Zeitpunkt der zweiten Untersuchung festgestellt werden $(p=0,5930)$. Die Anzahl an Patientinnen mit Akne sank unter Metformin von acht $(42,1 \%)$ auf sieben (36,8\%).

\subsubsection{Hirsutismus}

Zum Zeitpunkt der zweiten Untersuchung litten nur noch vier $(21,1 \%)$ anstatt acht von neunzehn (42,1\%) Patientinnen an Hirsutismus, was einem in der Tendenz verminderten Auftretens von Hirsutismus unter Metformin entsprach $(p=0,0679)$.

\subsubsection{Zyklusstörungen}

Im Vergleich zur Erstuntersuchung hatten sieben Patientinnen unter der Therapie mit Metformin wieder einen normalen Menstruationszyklus, was eine signifikante Reduktion der Zyklusstörungen um $36,84 \%$ bedeutete ( $p=0,0180$ ).

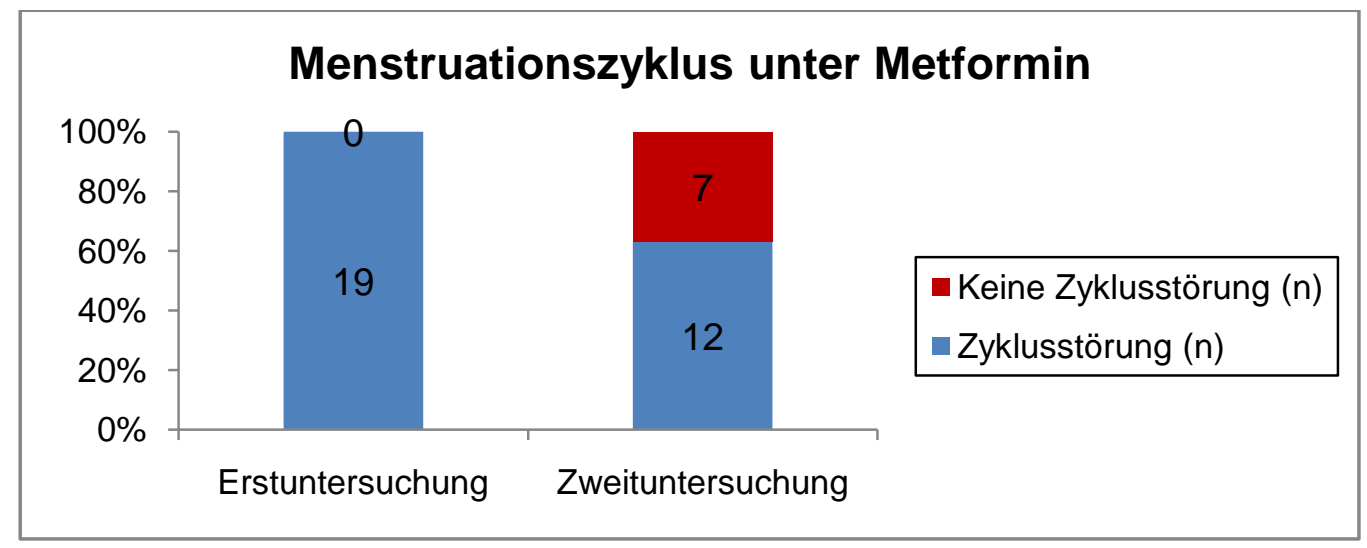

Tabelle 15: Auftreten von Zyklusstörungen zu beiden Untersuchungszeitpunkten. Bei mehr als einem Drittel der Patientinnen normalisierte sich der Menstruationszyklus unter der Therapie mit Metformin. 


\subsubsection{Ergebnisse des ersten und zweiten OGTT im Vergleich}

\subsubsection{Glukose, Insulin und C-Peptid}

Mit Ausnahme der Werte für Glukose nüchtern und nach einer Stunde sowie dem nüchtern Insulin-Wert ermittelte der t-Test für gepaarte Stichproben keine signifikanten Unterschiede für alle anderen Parameter.

Dabei ist auffällig, dass die Nüchternwerte von Glukose und Insulin sanken, zugleich jedoch die Glukosewerte nach 60 und 120 Minuten anstiegen.

\begin{tabular}{|c|c|c|c|c|c|c|c|c|}
\hline \multirow[b]{2}{*}{ Parameter } & \multirow[b]{2}{*}{ Norm } & \multicolumn{3}{|c|}{ Erstuntersuchung } & \multicolumn{3}{|c|}{ Zweituntersuchung } & \multirow[b]{2}{*}{ p-Wert } \\
\hline & & $\begin{array}{c}\text { Anzahl } \\
\text { (n) }\end{array}$ & $\begin{array}{l}\text { MW } \\
\pm \text { SD }\end{array}$ & Median & $\begin{array}{c}\text { Anzahl } \\
\text { (n) }\end{array}$ & $\begin{array}{l}\text { MW } \\
\pm \text { SD }\end{array}$ & $\begin{array}{l}\text { Media } \\
\quad \mathrm{n}\end{array}$ & \\
\hline $\begin{array}{c}\text { Glukose } \\
\text { nüchtern } \\
\text { (mg/dl) }\end{array}$ & $\begin{array}{l}N G T<90 \\
I G T<110 \\
D M \geq 200\end{array}$ & 30 & $\begin{array}{l}88,20 \\
\pm 9,07\end{array}$ & 90,00 & 30 & $\begin{array}{l}83,90 \\
\pm 9,81\end{array}$ & 84,00 & 0,0158 \\
\hline $\begin{array}{c}\text { Glukose } \\
1 \mathrm{~h} \\
\text { (mg/dl) }\end{array}$ & - & 30 & $\begin{array}{r}144,60 \\
\pm 29,07\end{array}$ & 143,50 & 30 & $\begin{array}{r}166,37 \\
\pm 44,82\end{array}$ & 162,00 & 0,0028 \\
\hline $\begin{array}{c}\text { Glukose } \\
2 \mathrm{~h} \\
\text { (mg/dl) }\end{array}$ & $\begin{array}{c}\text { NGT }<140 \\
\text { IGT:140-199 } \\
\text { DM } \geq 200\end{array}$ & 30 & $\begin{array}{r}112,17 \\
\pm 23,69\end{array}$ & 111,00 & 30 & $\begin{array}{r}125,93 \\
\pm 45,89\end{array}$ & 131,00 & 0,0721 \\
\hline $\begin{array}{c}\text { Insulin } \\
\text { nüchtern } \\
(\mu \mathrm{U} / \mathrm{ml})\end{array}$ & $<7$ & 30 & $\begin{array}{c}18,07 \\
\pm 11,85\end{array}$ & 16,20 & 30 & $\begin{array}{l}14,23 \\
\pm 7,81\end{array}$ & 12,35 & 0,0184 \\
\hline $\begin{array}{c}\text { Insulin } 1 \mathrm{~h} \\
(\mu \mathrm{U} / \mathrm{ml})\end{array}$ & - & 30 & $\begin{array}{c}154,70 \\
\pm \\
103,48\end{array}$ & 124,50 & 30 & $\begin{array}{r}144,95 \\
\pm 91,29\end{array}$ & 123,00 & 0,5079 \\
\hline $\begin{array}{c}\text { Insulin } 2 \text { h } \\
\qquad(\mu \mathrm{U} / \mathrm{ml})\end{array}$ & - & 30 & $\begin{array}{c}137,53 \\
\pm \\
124,90\end{array}$ & 96,05 & 30 & $\begin{array}{r}114,74 \\
\pm 98,42\end{array}$ & 90,10 & 0,1440 \\
\hline $\begin{array}{c}\text { C-Peptid } \\
\text { nüchtern } \\
(\mathrm{ng} / \mathrm{ml})\end{array}$ & $0,9-4,0$ & 30 & $\begin{array}{c}2,81 \\
\pm 0,97\end{array}$ & 2,60 & 30 & $\begin{array}{c}2,55 \\
\pm 0,93\end{array}$ & 2,20 & 0,1411 \\
\hline $\begin{array}{c}\text { C-Peptid } \\
1 \mathrm{~h} \\
(\mathrm{ng} / \mathrm{ml})\end{array}$ & - & 30 & $\begin{array}{r}10,63 \\
\pm 3,57\end{array}$ & 9,62 & 30 & $\begin{array}{l}11,66 \\
\pm 3,62\end{array}$ & 10,40 & 0,1353 \\
\hline $\begin{array}{c}\text { C-Peptid } \\
2 \mathrm{~h} \\
\text { (ng/ml) }\end{array}$ & - & 30 & $\begin{array}{l}10,18 \\
\pm 4,34\end{array}$ & 9,50 & 30 & $\begin{array}{l}11,09 \\
\pm 4,55\end{array}$ & 10,75 & 0,2342 \\
\hline
\end{tabular}

Tabelle 16: Ergebnisse beider OGTT. Für Insulin und C-Peptid existieren diesbezüglich noch keine Grenzwerte. $\mathrm{Zu}$ beiden Zeitpunkten bestand per Definition keine pathologische Glukosetoleranz. (Abkürzungen: NGT=Normale Glukosetoleranz; IGT=Gestörte Glukosetoleranz; DM=Diabetes mellitus) 


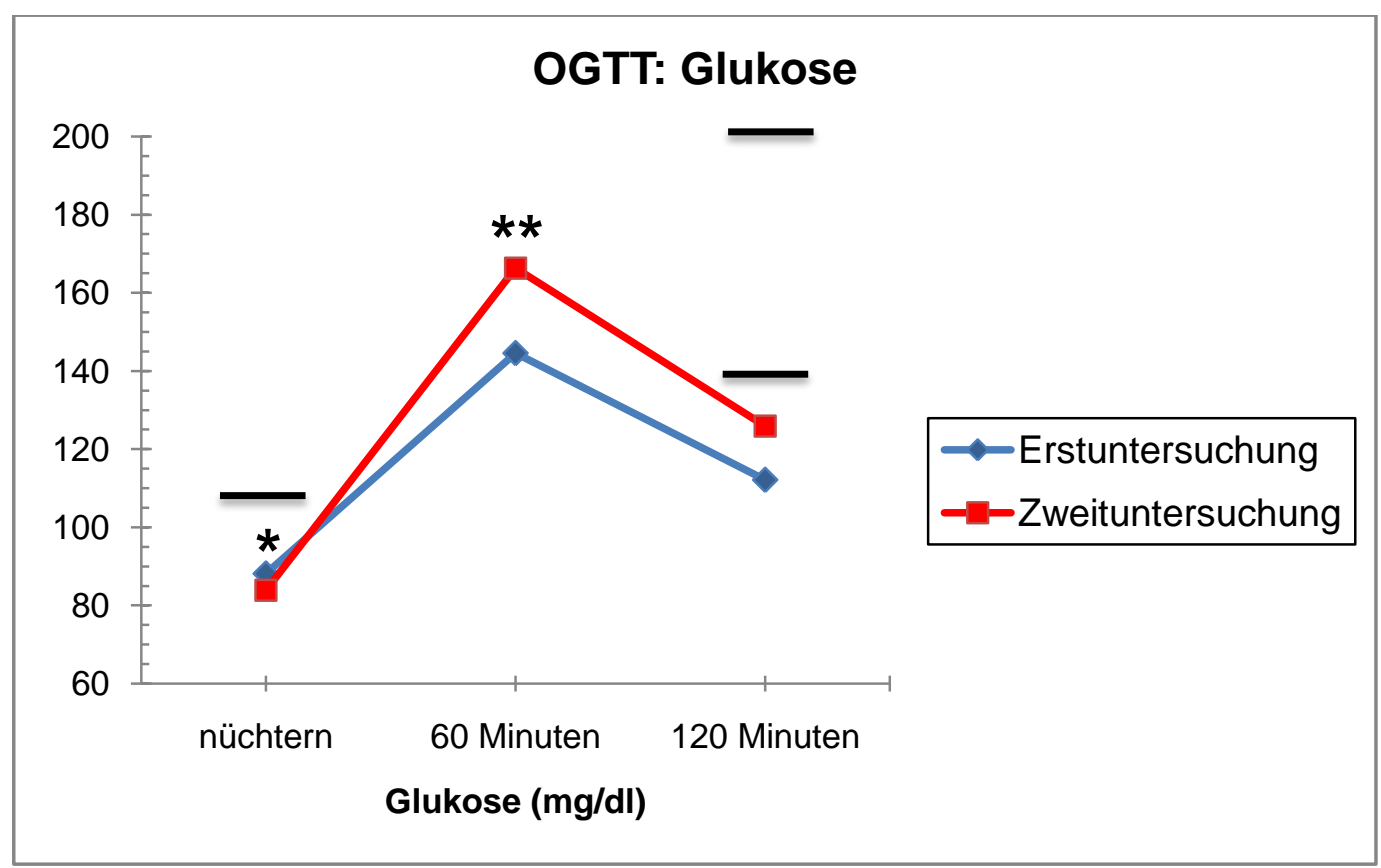

Abbildung 20: Mittelwerte von Glukose in beiden OGTT. Der zweite OGTT ergab für Glukose nach zwei Stunden einen tendenziell höheren Wert, nach einer Stunde einen signifikant höheren Wert. Dagegen war der Nüchternwert bei Erstuntersuchung signifikant niedriger. (Horizontale Balken nach Leitlinie der DDG, siehe Abschnitt 3.3.3.1: a) gestörte Glukosetoleranz: nüchtern>110 mg/dl und $2 \mathrm{~h}=140-199 \mathrm{mg} / \mathrm{dl}$. b) Diabetes mellitus: nüchtern $\geq 110 \mathrm{mg} / \mathrm{dl}$ und/oder $2 \mathrm{~h} \geq 200 \mathrm{mg} / \mathrm{dl}$ )

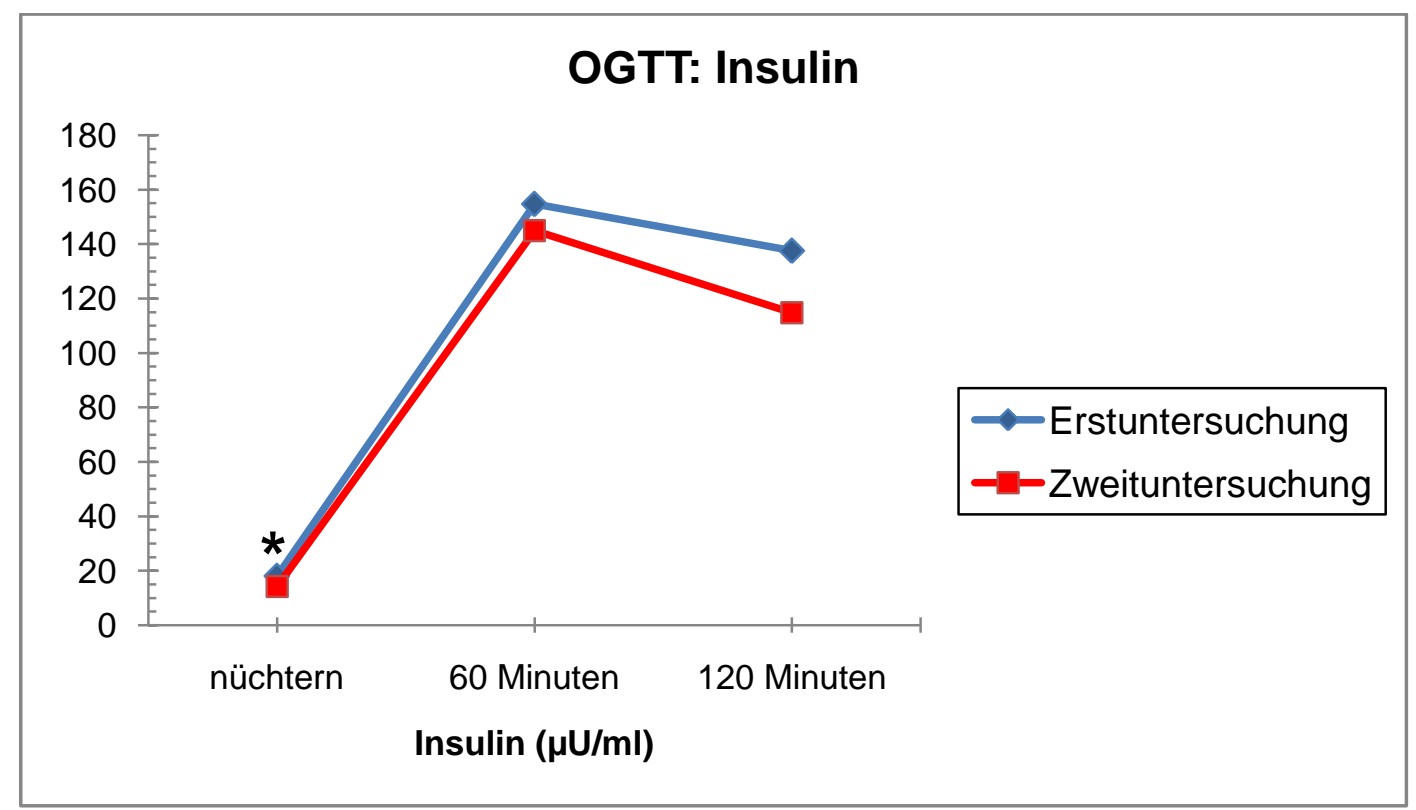

Abbildung 21: Darstellung der Insulin-Mittelwerte in beiden OGTT. Der zweite OGTT ergab einen signifikant niedrigeren Nüchternwert für Insulin. Derzeit existieren keine Standards zur klinischen Einschätzung der Hyperinsulinämie im OGTT; als Richtwerte für eine Hyperinsulinämie nach einer bis

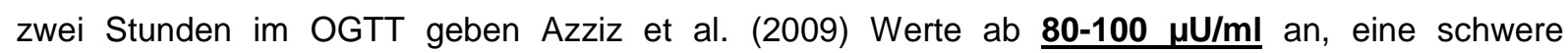
Hyperinsulinämie liege bei Werten $\geqq 300 \mu \mathrm{U} / \mathrm{ml}$ vor.] 


\subsubsection{AUC Glukose, AUC Insulin, AUC C-Peptid}

Bei keinem dieser drei Parameter zeigte sich ein signifikanter Unterschied hinsichtlich beider Untersuchungszeitpunkte. Allein die errechnete Fläche unter der Kurve von Glukose stieg in der Tendenz $(p<0,1)$ durchschnittlich um 13,43 mg/dl/min an.

\begin{tabular}{|c|c|c|c|c|c|c|c|}
\hline \multirow[b]{2}{*}{ Parameter } & \multicolumn{3}{|c|}{ Erstuntersuchung } & \multicolumn{3}{|c|}{ Zweituntersuchung } & \multirow[b]{2}{*}{ p-Wert } \\
\hline & $\begin{array}{l}\text { Anzahl } \\
\text { (n) }\end{array}$ & $\begin{array}{l}\text { MW } \\
\pm \text { SD }\end{array}$ & Median & $\begin{array}{l}\text { Anzahl } \\
\text { (n) }\end{array}$ & $\begin{array}{l}\text { MW } \\
\pm \text { SD }\end{array}$ & Median & \\
\hline $\begin{array}{c}\text { AUC Glukose } \\
\text { (mg/dl/min) }\end{array}$ & 30 & $\begin{array}{r}177,70 \\
\pm 28,72\end{array}$ & 174,50 & 30 & $\begin{array}{r}191,13 \\
\pm 47,63\end{array}$ & 190,00 & 0,0885 \\
\hline $\begin{array}{l}\text { AUC Insulin } \\
(\mu U / \mathrm{ml} / \mathrm{min})\end{array}$ & 30 & $\begin{array}{c}182,37 \\
\pm 134,71\end{array}$ & 140,50 & 30 & $\begin{array}{r}150,70 \\
\pm 79,23\end{array}$ & 127,00 & 0,1140 \\
\hline $\begin{array}{c}\text { AUC } \\
\text { C-Peptid } \\
\text { (ng/ml/min) }\end{array}$ & 30 & $\begin{array}{r}13,60 \\
\pm 4,95\end{array}$ & 12,50 & 30 & $\begin{array}{r}14,40 \\
\pm 4,24\end{array}$ & 14,00 & 0,3633 \\
\hline
\end{tabular}

Tabelle 17: Fläche unter der Kurve für Glukose, Insulin und C-Peptid jeweils im ersten und zweiten OGTT.

\subsubsection{HOMA-IR und QUICKI}

Der HOMA-IR sank signifikant ( $p=0,0118)$ im Durchschnitt um 0,97 auf 2,9 Punkte ( \pm 1,53 SD; Median: 2,80), wobei dies weiterhin über dem für eine wahrscheinliche Insulinresistenz gelegenen Cut-Off-Wert von 2,5 liegt. Der HOMA-IR war zu beiden Zeitpunkten nicht abhängig von der Einnahme eines Ovulationshemmers (siehe Abschnitt 3.4.5).

Der QUICKI stieg signifikant ( $p=0,0079)$ im Mittel um 0,01 auf 0,33 $( \pm 0,03$ SD, Median: 0,33) an, was einen geringeren Grad der Insulinresistenz bedeutet. 


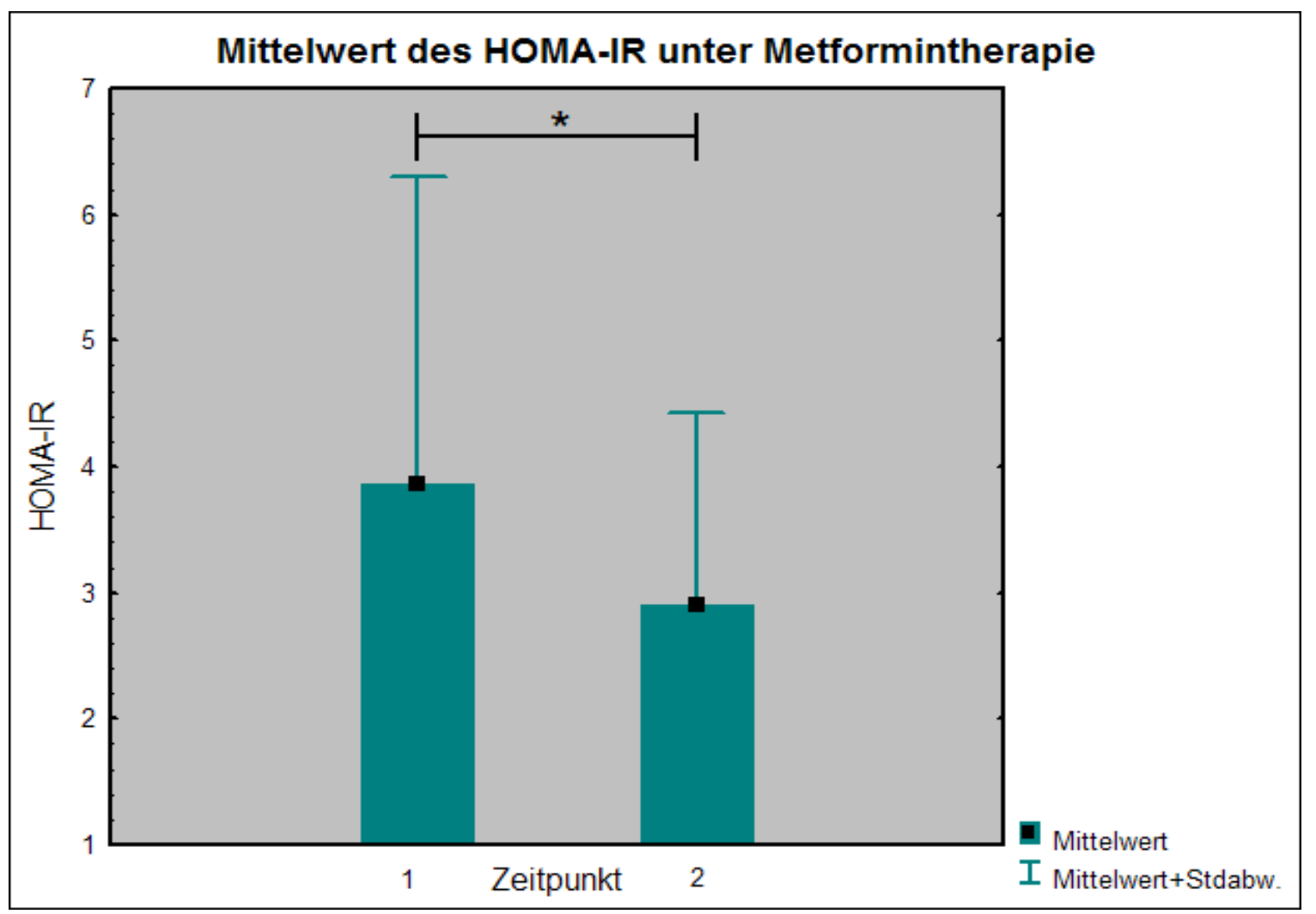

Abbildung 22: Mittelwert des HOMA-IR in beiden OGTT. Im zweiten OGTT errechnete sich ein signifikant niedrigerer HOMA-IR im Sinne verminderter Insulinresistenz.

\subsubsection{Einfluss von Ovulationshemmern}

Wie in Abschnitt 3.3.6 stellte sich auch in Gruppe 1 die Frage nach einem möglichen Einfluss von Ovulationshemmern auf bestimmte Parameter. Betrachtet wurden erneut der freie Androgenindex, HOMA-IR und die Fläche unter der Kurve von Insulin, wobei wiederum die pharmakologische Wirkungsweise des Ovulationshemmers nicht differenziert wurde.

Bei Erstuntersuchung hatten sechs Patientinnen (20\%) einen Ovulationshemmer in ihrer Vormedikation. Der zweiseitige t-Test für unabhängige Stichproben zeigte, dass der freie Androgenindex bei Patientinnen, die einen Ovulationshemmer einnahmen, signifikant niedriger war. SHBG-Spiegel waren unter Ovulationshemmer signifikant höher.

Zum Zeitpunkt der zweiten Untersuchung hatten elf Patientinnen (36,67\%) einen Ovulationshemmer in ihrer Vormedikation. Wiederum lag unter Einnahme eines Ovulationshemmers der freie Androgenindex signifikant niedriger und das SHBG signifikant höher. 


\begin{tabular}{|c|c|c|c|c|c|c|c|}
\hline \multirow[b]{2}{*}{ Parameter } & \multicolumn{3}{|c|}{ Ovulationshemmer } & \multicolumn{3}{|c|}{ Kein Ovulationshemmer } & \multirow[b]{2}{*}{ p-Wert } \\
\hline & $\begin{array}{c}\text { Anzahl } \\
\text { (n) }\end{array}$ & $\begin{array}{l}\text { MW } \\
\pm \text { SD }\end{array}$ & Median & $\begin{array}{c}\text { Anzahl } \\
\text { (n) }\end{array}$ & $\begin{array}{l}\text { MW } \\
\pm \text { SD }\end{array}$ & Median & \\
\hline $\begin{array}{l}\text { AUC Insulin } \\
(\mu \mathrm{U} / \mathrm{ml} / \mathrm{min})\end{array}$ & 6 & $\begin{array}{r}173,83 \\
\pm 66,27\end{array}$ & 147,50 & 24 & $\begin{array}{r}184.50 \\
\pm 148,00\end{array}$ & 140,00 & 0,7954 \\
\hline HOMA-IR & 6 & $\begin{array}{c}3,27 \\
\pm 1,22\end{array}$ & 3,10 & 24 & $\begin{array}{c}4,02 \\
\pm 2,65\end{array}$ & 3,75 & 0,3178 \\
\hline FAl & 6 & $\begin{array}{c}2,05 \\
\pm 2,66\end{array}$ & 1,05 & 24 & $\begin{array}{r}11,85 \\
\pm 8,27\end{array}$ & 11,30 & 0,0001 \\
\hline $\begin{array}{c}\text { BMI } \\
\left(\mathrm{kg} / \mathrm{m}^{2}\right)\end{array}$ & 6 & $\begin{array}{c}34,51 \\
\pm 5,73\end{array}$ & 33,58 & 24 & $\begin{array}{r}33,49 \\
\pm 5,08\end{array}$ & 34,42 & 0,7044 \\
\hline LH/FSH & 5 & $\begin{array}{c}1,25 \\
\pm 0,88\end{array}$ & 1,45 & 22 & $\begin{array}{r}1,92 \\
\pm 0,89\end{array}$ & 1,68 & 0,1743 \\
\hline $\begin{array}{c}\text { SHBG } \\
(\mathrm{nmol} / \mathrm{l})\end{array}$ & 6 & $\begin{array}{c}126,8 \\
\pm 54,91\end{array}$ & 139,00 & 24 & $\begin{array}{c}44,48 \\
\pm 44,66\end{array}$ & 30,95 & 0,0006 \\
\hline
\end{tabular}

Tabelle 18: Einfluss des Ovulationshemmers bei Erstuntersuchung. AUC Insulin, HOMA-IR, der BMI und LH/FSH-Quotient blieben von der Einnahme eines Ovulationshemmers unbeeinflusst.

\begin{tabular}{|c|c|c|c|c|c|c|c|}
\hline \multirow[b]{2}{*}{ Parameter } & \multicolumn{3}{|c|}{ Ovulationshemmer } & \multicolumn{3}{|c|}{ Kein Ovulationshemmer } & \multirow[b]{2}{*}{ p-Wert } \\
\hline & $\begin{array}{c}\text { Anzahl } \\
(\mathrm{n})\end{array}$ & $\begin{array}{l}\text { MW } \\
\pm \text { SD }\end{array}$ & Median & $\begin{array}{c}\text { Anzahl } \\
(\mathrm{n})\end{array}$ & $\begin{array}{l}\text { MW } \\
\pm \text { SD }\end{array}$ & Median & \\
\hline $\begin{array}{l}\text { AUC Insulin } \\
(\mu \mathrm{U} / \mathrm{ml} / \mathrm{min})\end{array}$ & 11 & $\begin{array}{r}142,82 \\
\pm 71,30\end{array}$ & 116,00 & 19 & $\begin{array}{r}155,26 \\
\pm 85,03\end{array}$ & 151,00 & 0,6719 \\
\hline HOMA-IR & 11 & $\begin{array}{c}2,97 \\
\pm 1,60\end{array}$ & 2,80 & 19 & $\begin{array}{c}2,86 \\
\pm 1,54\end{array}$ & 2,80 & 0,8494 \\
\hline $\begin{array}{c}\text { BMI } \\
\left(\mathrm{kg} / \mathrm{m}^{2}\right)\end{array}$ & 11 & $\begin{array}{r}32,07 \\
\pm 5,16\end{array}$ & 31,07 & 19 & $\begin{array}{l}32,21 \\
\pm 5,41\end{array}$ & 31,36 & 0,9451 \\
\hline FAl & 11 & $\begin{array}{r}1,77 \\
\pm 1,40\end{array}$ & 1,30 & 19 & $\begin{array}{c}9,77 \\
\pm 9,05\end{array}$ & 7,80 & 0,0012 \\
\hline $\begin{array}{c}\text { SHBG } \\
(\mathrm{nmol} / \mathrm{l})\end{array}$ & 11 & $\begin{array}{r}130,34 \\
\pm 47,80\end{array}$ & 131,00 & 19 & $\begin{array}{r}31,40 \\
\pm 15,69\end{array}$ & 29,70 & 0,00000 \\
\hline
\end{tabular}

Tabelle 19: Einfluss des Ovulationshemmers bei der zweiten Untersuchung. Wiederum blieben AUC Insulin, HOMA-IR und der BMI von der Einnahme eines Ovulationshemmers unbeeinflusst.

\subsubsection{FAl und SHBG}

Analog zu Abschnitt 3.3.7 wurden für das PCOS interessante Indizes ausgewählt, wobei der Einfluss von Ovulationshemmern berücksichtigt wurde. Im Gegensatz zur 
Erstuntersuchung wurde in den Folgeuntersuchungen nicht mehr der LH-FSHQuotient bestimmt, da dies lediglich der PCOS-Diagnostik diente.

\subsubsection{FAl}

Der Einfluss von Ovulationshemmern auf den freien Androgenindex war zu beiden Untersuchungszeitpunkten statistisch relevant, da jeweils die Patientinnen mit Ovulationshemmer einen signifikant niedrigeren FAI hatten. Aus diesem Grund wurden von 30 Patientinnen mit zweitem OGTT nur insgesamt 19 Patientinnen ohne Ovulationshemmer für die Auswertung des FAI berücksichtigt.

Unter Therapie mit Metformin sank der freie Androgenindex im Mittel um 1,8 auf 9,77 Punkte ( \pm 9,05 SD; Median: 7,80), was dennoch keinen signifikanten Abfall im Vergleich zur Erstuntersuchung darstellt ( $p=0,3324)$.

Betrachtet man bei der Auswertung alle 30 Patientinnen, sank der FAl im Mittel um 3,05 auf 6,84 Punkte ( \pm 8,18 SD; Median: 4,10), was einen signifikanten Abfall im Vergleich zur Erstuntersuchung war $(p=0,0381)$. Der Einfluss von Ovulationshemmern wird hierbei deutlich.

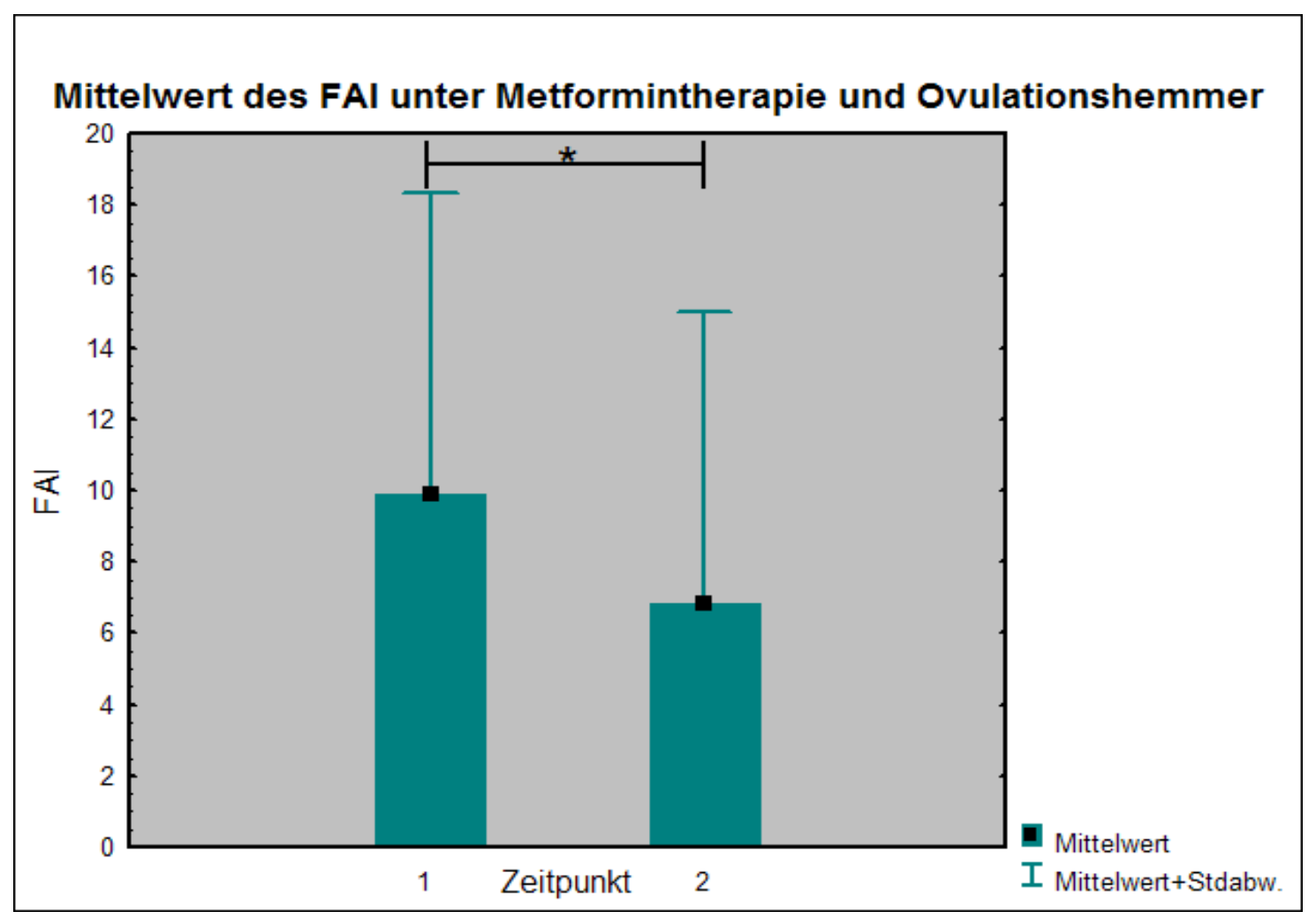

Abbildung 23: Mittelwert des FAl zu beiden Untersuchungszeitpunkten. Eine signifikante Abnahme wurde nicht erreicht, wenn Ovulationshemmer als Einflussgröße ausgeschlossen wurden. 


\subsubsection{SHBG}

Auch die SHBG-Spiegel waren statistisch signifikant von der Einnahme eines Ovulationshemmers beeinflusst, da jeweils die Patientinnen mit Ovulationshemmer einen signifikant höheren SHBG-Spiegel hatten.

Nach Auswertung von 19 Patientinnen ohne Ovulationshemmer in der Medikation ergab sich kein statistisch relevanter Unterschied der SHBG-Spiegel zu beiden Zeitpunkten (Erstuntersuchung: MW: 39,67 nmol/l $\pm 37,25 \mathrm{nmol} / \mathrm{I} \mathrm{SD;}$ Median: 33,20 nmol/l. Zweituntersuchung: MW: $31,40 \mathrm{nmol} / \mathrm{I} \pm$ 15,69 nmol/l SD; Median: 29,70 $\mathrm{nmol} / \mathrm{l})$.

Bei Auswertung aller 30 Patientinnen ergab sich trotz Ovulationshemmer kein signifikanter Anstieg bei insgesamt jedoch höheren SHBG-Spiegeln (Mittelwert bei Zweituntersuchung $67,68 \mathrm{nmol} / \mathrm{l} \pm 57,38 \mathrm{nmol} / \mathrm{l}$ ). 


\section{Diskussion}

Mit Blick auf die Fragestellung dieser Arbeit werden die Ergebnisse in den folgenden Abschnitten diskutiert. Zum besseren Verständnis der diskutierten Ergebnisse folgt jedoch zunächst ein Einschub zum Einfluss von Ovulationshemmern auf die untersuchten Parameter.

\subsection{Einfluss von Ovulationshemmern}

In dieser Studie nahmen einige Patientinnen einen Ovulationshemmer ein. Die Ergebnisse wurden somit unter Berücksichtigung des Einflusses von Ovulationshemmern auf bestimmte Parameter interpretiert. Im Hinblick auf die pathophysiologische Wirkung eines oralen Ovulationshemmers - es wurde jedoch nicht nach Ein-/Mehrphasen-Präparat, sog. Minipille oder antiandrogenem Ovulationshemmer unterschieden - gehen die meisten Ergebnisse mit gegenwärtigen Erkenntnissen konform: Unter Einnahme eines Ovulationshemmers war der freie Androgenindex signfikant niedriger sowie die Spiegel des SHBG signifikant höher als bei Patientinnen ohne Ovulationshemmer.

\section{Ovulationshemmer}

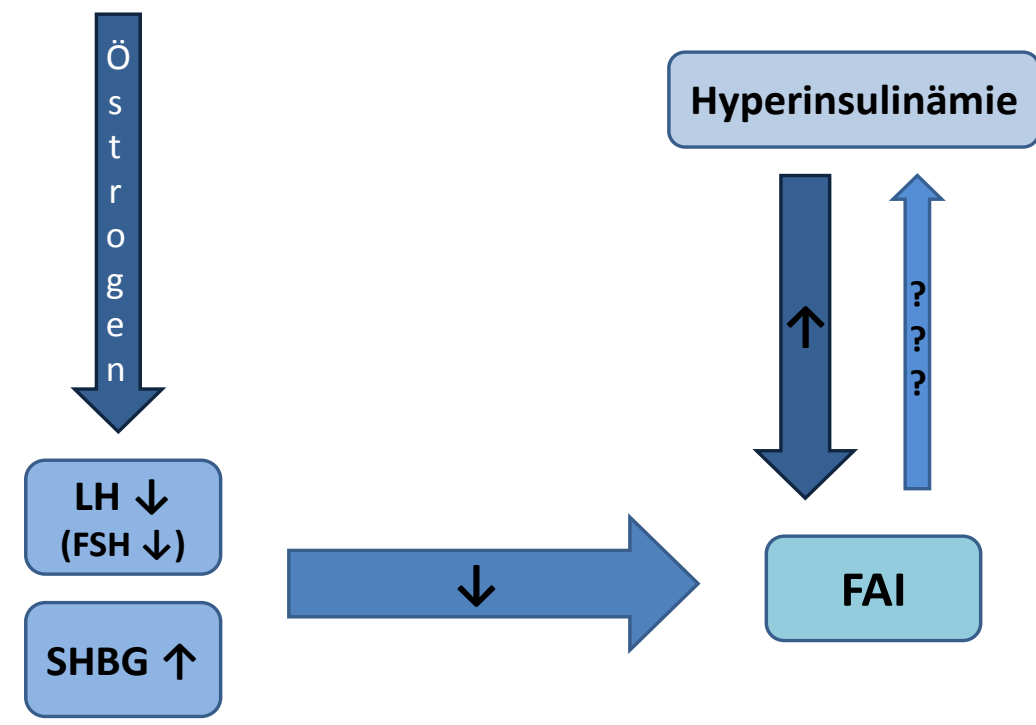

Abbildung 24: Einfluss von Ovulationshemmern und Hyperinsulinämie auf den FAl. Hyperinsulinämie führt zu erhöhten Androgenen. Es ist jedoch noch unklar, ob erhöhte Androgene auch zu einer Hyperinsulinämie führen. 
Unklar bleibt der Einfluss auf metabolische Parameter. Orale Ovulationshemmer gehören, wie auch Diuretika aus der Gruppe der Thiazide, zu einer Reihe von Medikamenten mit potenziell verschlechternder Wirkung auf die Glukosetoleranz (O'Byrne und Feely 1990a; O'Byrne und Feely 1990b). Eine aktuelle Metaanalyse von Halperin et al. (2011) ergab größtenteils keinen statistisch relevanten Einfluss von Ovulationshemmern auf metabolische Parameter. Bei der Nüchternglukose, dem Nüchterninsulin, dem HOMA-IR und überdies auch dem euglykämischhyperinsulinämischen Clamp-Verfahren bestand kein signifikanter Zusammenhang mit der Einnahme von Ovulationshemmern. Lediglich Triglyzeride und HDLCholesterin stiegen hierunter signifikant an. Die Autoren der Metaanalyse verweisen jedoch auf die begrenzte Aussagekraft inrer Ergebnisse auf Grund großer Heterogenität der Kohorten in den ausgewählten Studien, und weisen auf den Bedarf an randomisierten, kontrollierten Studien zur Bestätigung dieser Ergebnisse. Eine nachträgliche Auswertung des HDL-Cholesterins erbrachte ein nach der Metanalyse erwartetes Ergebnis: Beide Gruppen, sowohl PCOS-Patientinnen als auch Patientinnen ohne PCOS, hatten unter der Medikation mit einem Ovulationshemmer ein signifikant höheres HDL-Cholesterin als Patientinnen ohne Ovulationshemmer.

Es wurden letztlich nur ausgewählte Parameter hinsichtlich eines solchen Einflusses berücksichtigt, da eine umfassende Auswertung aller Parameter den inhaltlichen Rahmen dieser Arbeit überstiegen hätte. Die entsprechenden Parameter werden in den folgenden Abschnitten diskutiert.

Schlussendlich wäre ein Vergleich der Parameter vor und nach Einnahme eines Ovulationshemmers hilfreicher für die Frage gewesen, ob ein Einfluss auf verschiedene Parameter besteht. Die Daten wurden jedoch nicht entsprechend generiert.

\subsection{Vergleich der Gruppen PCOS und Ausschluss PCOS}

\subsubsection{Alter und BMI}

In den klinischen Basisparametern Alter und BMI konnten Unterschiede in den beiden Gruppen PCOS und Ausschluss PCOS dargestellt werden. PCOSPatientinnen waren in dieser Studie durchschnittlich sieben Jahre und damit signifikant jünger als die Patientinnen mit im Verlauf ausgeschlossenem PCOS. 
Dagegen konnte nur eine Tendenz für einen erhöhten BMI der PCOS-Patientinnen gezeigt werden, obwohl dieser im Gegensatz zu Patientinnen ohne PCOS durchschnittlich im adipösen Bereich lag. In dieser Studie hatten 64,7\% der PCOSPatientinnen einen $\mathrm{BMI} \geq 30 \mathrm{~kg} / \mathrm{m}^{2}$; in der Vergleichsgruppe waren es nur $43,1 \%$.

Diese Ergebnisse sind konform mit den Ergebnissen zahlreicher Studien zu erhöhter Prävalenz von Adipositas bei PCOS-Patientinnen im Vergleich zu Kontrollpatientinnen ohne PCOS. Die Prävalenz unterliegt dabei regionalen Unterschieden; in den USA werden im Kontext des allgemeinen Bevölkerungswandels die höchsten Raten an adipösen PCOS-Patientinnen erzielt (Azziz et al. 2009). So stieg der Anteil an adipösen PCOS-Patientinnen innerhalb von 15 Jahren von 51 auf 74 \% (Yildiz et al. 2008). Umgekehrt lässt sich bei bis zu 28,3\% der übergewichtigen und adipösen Patientinnen in Spanien ein PCOS diagnostizieren (Alvarez-Blasco et al. 2006). In Studien mit Kollektiven deutscher PCOS-Patientinnen hatten bei Hahn et al. (2004) $62 \%$ der Patientinnen einen $B M l \geq 25 \mathrm{~kg} / \mathrm{m} 2$ sowie bei Tan et al. (2007) $54,2 \%$ der Patientinnen einen BMI $\geq 30$ $\mathrm{kg} / \mathrm{m}^{2}$. Die Patientinnen der vorliegenden Studie sind mit beiden letztgenannten deutschen Studien hinsichtlich ihrer klinischen Basisparameter vergleichbar.

\subsubsection{Akne, Hirsutismus und Zyklusstörungen}

Anhand der klinischen PCOS-Parameter Hirsutismus und Zyklusstörungen konnten in dieser Studie die PCOS-Patientinnen klar von Patientinnen ohne PCOS unterschieden werden. Patientinnen mit PCOS litten signifikant häufiger an Zyklusstörungen als Patientinnen ohne PCOS (83,33 \% zu 32,56 \%). Hirsutismus fand sich signifikant häufiger bei Patientinnen mit PCOS, wobei die Prävalenz mit lediglich 48,61\% deutlich niedriger als erwartet ausfiel.

Die Prävalenz der Zyklusstörungen entsprach der aktuellen von Azziz et al. (2009) analysierten Studienlage, wonach 75 bis $85 \%$ der PCOS-Patientinnen keinen normalen Menstruationszyklus haben. Es werden dabei noch höhere Prävalenzen vermutet, da nicht jede Frau mit derartigen Beschwerden eines potenziellen PCOS medizinische Hilfe in Anspruch nimmt. Azziz et al. (2009) gaben nach Analyse der Studien eine Prävalenz von 65 bis $75 \%$ für Hirsutismus beim PCOS in der schwarzen, weißen und südostasiatischen Bevölkerung an, im Gegensatz zu den bei 
uns 48,61 \%. Unterschiedliche Prävalenzen resultieren nicht nur aus der betrachteten Ethnie - Patientinnen in Ostasien leiden seltener unter Hirsutismus -, sondern auch aus den gewählten Definitionskriterien des PCOS und verschiedenen Cut-off-Werten des Ferriman-Gallway-Score. So gibt Lowenstein (2006) eine Prävalenzspanne von 40 bis $92 \%$ bei europäischen und amerikanischen Frauen mit PCOS an. In einer Studie mit deutschen PCOS-Patientinnen litten 64,5\% der Frauen unter Hirsutimus (Hahn et al. 2005).

Die Prävalenz von Hirsutismus bei PCOS-Patientinnen lag in dieser Studie im unteren Erwartungs- aber der Studienlage entsprechend noch im Normbereich. Der niedrigere Wert könnte eventuell durch die nicht standardmäßige Anwendung des Ferriman-Gallway-Score bedingt sein, da die Daten sich letztlich in vielen Fällen nur auf anamnestische Angaben aus Untersuchungsbögen und Arztbriefen stützen.

In Bezug auf das Auftreten von Akne unterschieden sich beide Gruppen nicht von einander. Die Prävalenz lag in beiden Gruppen unter $30 \%$.

Azziz et al. (2009) geben mit 15 bis $25 \%$ eine sogar noch niedrigere Prävalenz von Akne bei PCOS-Patientinnen an. Diese variiert wiederum je nach Ethnie und PCOSKriterien, und liegt offenkundig häufiger bei jüngeren (PCOS-)Patientinnen vor. Hahn et al. (2005) diagnostizierten eine Akne bei $35 \%$ der deutschen PCOS-Patientinnen. Im Umkehrschluss kann bei einer hohen Zahl an Patientinnen (74\%) mit schwerer Akne in der Folge ein PCOS diagnostiziert werden (Lowenstein 2006).

Die vorliegende Studie zeigte, dass das Auftreten von Akne als klinische Manifestation einer Hyperandrogenämie gewertet werden kann, aber mangels geringer Spezifität eher unerheblich in der Diagnostik des PCOS ist. Auch bleibt das durch ein PCOS erhöhte Risiko für die Entwicklung einer Akne unklar (Azziz et al. 2009).

Die androgenetische Alopezie wurde in dieser Studie nicht standardmäßig anhand des Ludwig-Score untersucht bzw. lediglich in Einzelfällen in Arztbriefen vermerkt, so dass dieser klinische Parameter nicht erfasst wurde. Azziz et al. (2009) geben eine große Prävalenzspanne von 5 bis $50 \%$ bei PCOS-Patientinnen an. In einer deutschen Studie lag die Prävalenz bei $31 \%$ (Hahn et al. 2005). Ähnlich der Akne verbleibt der androgenetischen Alopezie derzeit nur der Status als unspezifischer 
Indikator einer Hyperandrogenämie, ohne große bzw. mit unklarer diagnostischer Wertigkeit für das PCOS.

\subsubsection{Hashimoto-Thyreoiditis (AIT)}

Ein signifikant höheres Auftreten der autoimmunen Hashimoto-Thyreoiditis (AIT) bei PCOS-Patientinnen konnte nicht bestätigt werden. 13,73 \% der PCOS-Patientinnen in dieser Studie hatten eine AIT. Dies steht teilweise im Widerspruch zu einigen Studien, die ein gehäuftes Auftreten der AIT bei PCOS-Patientinnen zeigten. Janssen et al. (2004) konnten in der bis dato ersten multizentrischen Studie zu Schilddrüsenfunktionsstörungen bei PCOS-Patientinnen eine dreifach höhere Prävalenz der AIT nachweisen (20,6 \% vs. 6,5\%). PCOS-Patientinnen hatten eine im Vergleich zu gesunden Frauen höhere Prävalenz bei allen spezifischen Parametern (Autoantikörper gegen TPO und TG, sonographische Hypoechogenität und Schilddrüsenvolumen). Eine aktuelle Studie belegt die höhere Prävalenz der AIT bei PCOS-Patientinnen (nach den Rotterdamkriterien), wobei nur die TPOAutoantikörper und eine palpierte (nicht sonographierte) Struma häufiger auftraten; die TSH-Spiegel waren nicht unterschiedlich (Kachuei et al. 2011). Hingegen beschritten Ganie et al. (2010) den umgekehrten Weg, und bewiesen eine höhere Prävalenz des PCOS (nach den Rotterdamkriterien) bei Patientinnen mit euthyreoter chronisch lymphatischer Thyreoiditis (46,8\%), welche auch einen signifikant höheren BMI und HOMA-IR als die Kontrollgruppe hatten. In einer Übersicht der Androgen Excess Society (Azziz et al. 2009) wird von einer eher niedrigen Prävalenz allgemeiner thyreoidaler Störungen bei PCOS-Patientinnen ausgegangen; bezugnehmend zu den oben präsentierten Ergebnissen von Janssen et al (2004) werden weitere Studien empfohlen.

Ein möglicher Grund für das von der Studienlage abweichende Ergebnis dieser Studie ist die gewählte Zuordnung der Patientinnen zu den Gruppen. Es kann vermutet werden, dass die nach den Rotterdamkriterien ausgeschlossenen Patientinnen dennoch eine Art graduelles PCOS aufweisen, so dass nach aktueller Leitlinie der Androgen Excess Society (Azziz et al. 2006) bei einigen Patientinnen mitunter ein PCOS vorliegt. Behält man die gewählte Trennung der Gruppen bei, kann die Hypothese aufgestellt werden, dass in beiden Gruppen eine in Teilen 
gemeinsame Pathophysiologie existiert, welche mit dem Auftreten einer autoimmunen Hashimoto-Thyreoiditis korreliert. Dies ist der Vermutung von Janssen et al. (2004) entlehnt, wonach auf Grund der Beobachtung, dass beim PCOS gehäuft andere autoimmune Erkrankungen vorliegen, das PCOS und die AIT einen gemeinsamen genetischen Hintergrund im Sinne einer gemeinsamen autoimmunen Genese haben. Weiterhin kann auch die zur Auswertung verwendete nominale „ja/nein“-Skala zum Vorliegen einer AIT einen Grund für das abweichende Ergebnis der vorliegenden Studie darstellen. Die Diagnose wurde lediglich aus Arztbriefen und Anamnesebögen verschiedener Behandler übernommen; bei Datenerhebung wurden die einzelnen Parameter nicht wie bei Janssen et al. (2004) einzeln ausgewertet. Schließlich kann die Tatsache, dass beide Gruppen heterogen bezüglich des Alters waren, ein möglicher Erklärungsansatz für das gefundene Ergebnis sein. Das Alter der PCOS-Patientinnen lag mit 26 Jahren etwa in der Mitte des Altersdurchschnitts der Studien von Janssen et al. (2004) und Kachuei et al. (2011).

In Übereinstimmung mit den Empfehlungen dieser beiden Studien kann bei Bewertung der vorliegenden Untersuchung die routinemäßige SchilddrüsenDiagnostik - inklusive spezifischer Autoantikörper - bei PCOS-Patientinnen empfohlen werden, obwohl die Evidenz zum gehäuften Auftreten einer AIT beim PCOS noch unklar ist.

\subsubsection{LH/FSH}

In beiden Gruppen war der LH/FSH-Quotient durch Ovulationshemmer unbeeinflusst. PCOS-Patientinnen hatten in dieser Studie einen signifikant höheren LH/FSHQuotienten, welcher mit 1,64 unter dem für das PCOS typischen Wert von $\geq 2,0$ aber dennoch über dem im Zyklus gemittelten Normwert von 1,0 lag. Lediglich bei adipösen PCOS-Patientinnen $\left(\mathrm{BMl} \geq 30 \mathrm{~kg} / \mathrm{m}^{2}\right)$ und bei normalgewichtigen Patientinnen ohne PCOS $\left(\mathrm{BMl}<25 \mathrm{~kg} / \mathrm{m}^{2}\right)$ bedingte der BMI die Höhe des $\mathrm{LH} / \mathrm{FSH}$ Quotienten. Eine negative Korrelation zwischen Alter und LH/FSH-Quotienten bestand statistisch signifikant bei Patientinnen ohne PCOS, bei PCOS-Patientinnen noch in der Tendenz.

Seit mehr als 40 Jahren sind Störungen der Gonadotropine beim PCOS bekannt (Azziz et al. 2009). In zahlreichen Studien konnte belegt werden, dass PCOS- 
Patientinnen eine erhöhte Frequenz und Amplitude der LH-Ausschüttung aufweisen (Morales et al. 1996; Taylor et al. 1997; Waldstreicher et al. 1988). Pathophysiologisch wird dabei eine erhöhte GnRH-Pulsfrequenz vermutet, die unphysiologisch hohe LH-Werte im Plasma zur Folge hat (Blank et al. 2006). Morales et al. (1996) zeigten, dass die Frequenz der LH-Ausschüttung bei dünnen (BMI $\leq 23$ $\left.\mathrm{kg} / \mathrm{m}^{2}\right)$ und adipösen $\left(B M \mathrm{l} \geq 30 \mathrm{~kg} / \mathrm{m}^{2}\right)$ PCOS-Patientinnen, die Pulsamplitude der LHAusschüttung jedoch nur bei dünnen PCOS-Patientinnen anstieg. Der resultierende LH-Anstieg im Plasma wäre demnach unabhängig von vorliegender Adipositas ein Charakteristikum des PCOS. Zu ähnlichen Ergebnissen kamen auch Pagán et al. (2006), die in inrer Studie den Einfluss des BMI in Form einer negativen Korrelation auf den LH/FSH-Quotienten sowie auf die Pulsamplitude der LH-Ausschüttung darstellten. Der BMI hatte dabei keinen Einfluss auf die hypothalamische Funktion, beeinflusste somit nicht die Frequenz der LH-Ausschüttung via GnRH. Srouji et al. (2007) bestätigten die inverse Beziehung zwischen BMI und LH durch eine veränderte Pharmakokinetik von endogenem $\mathrm{LH}$ bei steigendem $\mathrm{BMI}$, da rekombinantes humanes LH keine pharmakokinetischen Veränderungen zeigte.

Die vorliegende Arbeit konnte erhöhte LH/FSH-Quotienten bei PCOS-Patientinnen im Vergleich zu Patientinnen ohne PCOS bestätigen. Im Kontrast zu den aufgeführten Studien resultierte jedoch keine negative Korrelation zwischen BMI und LH/FSH-Quotient bei PCOS-Patientinnen. Diente hierfür und für das Nichterreichen des für das PCOS typischen LH/FSH-Quotienten $\geq 2,0$ zuerst noch der hohe Anteil an adipösen PCOS-Patientinnen $(64,7 \%)$ als Erklärungsansatz, konnte dies bei nachträglichen Subanalysen nicht mehr aufrecht erhalten werden. Das Kollektiv beider Gruppen und insbesondere das der PCOS-Patientinnen war bei Betrachtung dieses Parameters höchstwahrscheinlich zu heterogen, um ein Ergebnis ähnlich der oben genannten Studien zu zeigen. PCOS-Patientinnen waren beispielsweise bedeutend jünger. Es handelte sich zudem nicht um ein prospektiv geplantes Studienkollektiv, sondern vielmehr um Versorgungsforschung im Sinne einer Routineversorgung der PCOS-Patientinnen in der Praxis. Weiterhin hatten Ovulationshemmer zwar statistisch keinen Einfluss auf den LH/FSH-Quotienten, aber die fehlende Differenzierung nach verschiedenen pharmakologischen Arten der Ovulationshemmer erschwert eine valide Aussage zu diesem Aspekt. Hieran fügt sich noch die Tatsache, dass bei Datenerhebung keine sichere Aussage getroffen 
werden konnte, in welcher Phase des Menstruationszyklus die Blutentnahme statt fand, da oft auch eine Amenorrhoe vorlag. Die Laborwertbestimmung sollte standardmäßig am dritten bis fünften Zyklustag bzw. nach Ovulationsinduktion bei Oligo-/Amenorrhoe erfolgen (siehe Abschnitt 1.4.4, S. 8). Daher wäre der niedrigere LH/FSH-Quotient auch im Kontext variabler Zeitpunkte der Laborbestimmung zu erklären. Dies könnte bei zukünftiger Interpretation von Laborwerten im Endokrinologikum Berücksichtigung finden.

\subsubsection{SHBG}

Patientinnen hatten in beiden Gruppen bei Einnahme eines Ovulationshemmers deutlich höhere SHBG-Spiegel als Patientinnen ohne Ovulationshemmer in der Vormedikation, was zum Ausschluss dieser Patientinnen für die Auswertung führte. Die Werte des SHBG zeigten keinen Unterschied in beiden Gruppen, und lagen im niedrignormalen Referenzbereich für Frauen. Das SHBG war somit nicht, wie für das PCOS charakteristisch, erniedrigt.

Dass Ovulationshemmer die SHBG-Synthese induzieren, wurde in zahlreichen Studien nachgewiesen (Key et al. 1989; Panzer et al. 2006). Ebenso wurde das beim PCOS typischerweise erniedrigte SHBG in vielen Studien nachgewiesen, und gilt gegenwärtig als ein Charakteristikum des PCOS (Azziz et al. 2009; Chen et al. 2010).

Warum in der vorliegenden Studie kein signifikanter Unterschied in beiden Gruppen hinsichtlich der Höhe des SHBG festgestellt werden konnte, liegt möglicherweise an der bereits oben angeführten Zuordnung der Patientinnen zu den Gruppen. Pathophysiologische Gemeinsamkeiten von PCOS-Patientinnen und mit nach den Rotterdam-Kriterien ausgeschlossenen Patientinnen sind auch hierbei im Bereich des Möglichen, zumal beide Gruppen ohne Ovulationshemmer ein eher niedrignormales SHBG aufwiesen. Weiterhin unterschieden sich beide Gruppen auch unter Berücksichtigung der Einnahme eines Ovulationshemmers signifikant im Alter. Der Altersunterschied von sieben Jahren wurde in der Auswertung nicht mit einbezogen. Folglich war in dieser Studie eine klare Trennung der beiden Gruppen anhand des SHBG nicht möglich. 
Das SHBG war in dieser Studie in beiden Gruppen nicht mit dem HOMA-IR und Nüchterninsulin korreliert. Eine negative Korrelation zwischen der Fläche unter der Kurve von Insulin (AUC Insulin) und dem SHBG lag allein bei Patientinnen ohne PCOS vor, nicht jedoch bei PCOS-Patientinnen. Hingegen bestand in beiden Gruppen eine negative Korrelation zwischen BMI und SHBG, wenn auch bei PCOSPatientinnen lediglich in der Tendenz.

Dem SHBG wird in Studien gegenwärtig die Rolle eines zuverlässigen Markers der Insulinresistenz und die eines Prädiktors von Diabetes mellitus Typ 2 zugewiesen. Schüring et al. (2008) gehen davon aus, dass erhöhte Insulinwerte im Pfortaderkreislauf zirkulierende SHBG-Spiegel verringern, und auf diesem Wege indirekt das freie Testosteron ( $F A l$ ) erhöhen (siehe unten). In einer älteren Studie stellten Pugeat et al. (1996) das SHBG als vielseitigen diagnostischen Parameter dar; unter anderem auch als indirekten Marker der Insulinresistenz und kardiovaskulärem Risiko bei postmenopausalen Frauen. Bei Patientinnen mit Hirsutismus war das SHBG neben insgesamt niedrigen SHBG-Werten negativ mit dem Nüchterninsulin und dem BMI korreliert; eine positive Korrelation ergab sich mit dem HDL-Cholesterin. Die Untersuchungen von Gannagé-Yared (2011) zum androgenen Hormonprofil (mit SHBG) und metabolischen Parametern (unter anderem BMI, HOMA-IR) bei jungen, gesunden Männern und Frauen ergaben für beide Geschlechter signifikante, negative Korrelationen. Unter Berücksichtigung von Störgrößen (BMI, Bauchumfang, Alter, Nikotinabusus) war jedoch das SHBG nur noch bei Männern negativ mit dem HOMA-IR korreliert. Die elfjährige follow-upBeobachtung der Pizzara-Studie kam zum Ergebnis, dass niedrige SHBG-Spiegel bei beiden Geschlechtern das Risiko einer Insulinresistenz und in der Folge auch Diabetes mellitus erhöhen (Soriguer et al. 2012). Bei PCOS-Patientinnen konnte auch der Zusammenhang von präkonzeptionell niedrigem SHBG und der Entwicklung eines Gestationsdiabetes gezeigt werden. Unter Berücksichtigung der geringen Stichprobengröße wird der prädiktive Wert des SHBG größer als der des BMI, Bauchumfang oder HOMA-IR eingeschätzt (Veltman-Verhulst et al. 2010).

Das SHBG war in dieser Studie nicht als Indikator einer Insulinresistenz anhand des HOMA-IR und Nüchterninsulins geeignet. In der Zusammenschau der Studien ergeben sich Ansätze, die den fehlenden Zusammenhang von SHBG und Insulinresistenz erklären könnten. Zum einen wurden die Gruppen nicht nach 
möglichen Störgrößen wie dem Alter und auch dem Bauchumfang differenziert; wie bereits erwähnt waren PCOS-Patientinnen jünger und der Bauchumfang wurde nicht erhoben. Gannagé-Yared et al. (2011) zeigten zudem, dass es eine Korrelation mit dem HOMA-IR nur bei Männern gab. Das in dieser Arbeit untersuchte Kollektiv war auch für den Parameter SHBG zu heterogen, um sich den Ergebnissen oben genannter Studien anzuschließen, wobei die dort untersuchten Kollektive nicht durchweg homogen und vergleichbar waren.

\subsubsection{FAl}

Auch der freie Androgenindex wurde durch die Einnahme eines Ovulationshemmers beeinflusst. In beiden Gruppen hatten Patientinnen mit Ovulationshemmer einen signifikant niedrigeren FAI. Der FAI war bei PCOS-Patientinnen signifikant höher als bei Patientinnen mit ausgeschlossenem PCOS. Obwohl die Mittelwerte in beiden Gruppen über dem für Frauen geltenden Cut-Off-Wert von 3,5 lagen, war der Anteil der Patientinnen mit einem FAI $\geq 3,5$ in der PCOS-Gruppe deutlich höher (87,14\% vs. $46,51 \%)$.

Die Hyperandrogenämie ist wie in den Abschnitten 1.2 und 1.3 (S. 1 ff.) dargestellt ein zentraler Aspekt in der Pathophysiologie und Klinik des PCOS. Azziz et al. (2009) schlussfolgern in ihrem task-force-Report zur Diagnose des PCOS, dass die Messung des freien Testosterons sensitiver als die Messung des gesamten Testosterons im Hinblick auf die Diagnose hyperandrogenetischer Störungen ist. Die direkte Messung des freien Testosterons sei dennoch mit den gegenwärtig verfügbaren Assays schlecht reproduzierbar, so dass der aus Gesamttestosteron und dem SHBG errechnete freie Androgenindex die bessere Variante darstelle. So werden in $70 \%$ der nach den NIH-Kriterien von 1990 (Carmina 2004) diagnostizierten PCOS-Patientinnen erhöhte Werte des FAI beobachtet. Zudem sollten auch andere Androgene gemessen werden, wenn 20 bis $30 \%$ der PCOSPatientinnen normale Testosteronwerte aufweisen. In einer aktuelleren Studie von Huang et al. (2010) mit 720 ebenfalls nach den NIH-Kriterien definierten PCOSPatientinnen hatten $75,3 \%$ eine Hyperandrogenämie; die Prävalenz eines gemessenen und nicht errechneten, erhöhten FAl lag bei 57,5 \% (Huang et al. 2010). Hahn et al. (2005) beobachteten in einem Kollektiv deutscher PCOS-Patientinnen bei 
$82 \%$ erhöhte Testosteronwerte, ohne dabei den FAl zu berechnen. Eine Langzeitstudie mit nur 34 PCOS-Patientinnen konnte zeigen, dass es im Verlauf der Jahre zu abnehmenden Werten des FAI bei steigenden Werten des SHBG kommt (Hudecova et al. 2011).

Die Ergebnisse dieser Studie zum FAl entsprechen den oben aufgeführten aktuellen Erkenntnissen. Die alleinige Betrachtung des Mittelwertes - in beiden Gruppen über dem Normwert für Frauen - lässt die Schlussfolgerung zu, dass beide Gruppen in dieser Studie per se hyperandrogenämisch sind, was auf den bereits mehrfach erwähnten pathophysiologischen Gemeinsamkeiten fußen könnte. Dennoch sei das Argument angeführt, dass im Vergleich erheblich mehr PCOS-Patientinnen über dem Normwert des FAI von $\geq 3,5$ liegen.

Eine positive Korrelation zwischen der Fläche unter der Kurve (AUC) von Insulin und dem freien Androgenindex konnte in beiden Gruppen nachgewiesen werden. Der Zusammenhang zwischen dem HOMA-IR und dem FAI war lediglich bei PCOSPatientinnen statistisch signifikant.

Hyperinsulinämie führt auf verschiedenen Wegen zu erhöhten Werten des FAl, was in zahlreichen Studien beobachtet wurde (siehe Abschnitt 1.3.2, S. 3 und Abb. 1, S. 5). Acién et al. (1999) zeigten signifikante Korrelationen zwischen Insulinresistenz und Hyperandrogenämie (gemessen als freies Testosteron) sowohl bei Patientinnen mit PCOS als auch bei Patientinnen ohne PCOS. Mit zunehmender Adipositas verstärkte sich diese Korrelation nur bei PCOS-Patientinnen. Im Umkehrschluss konnten Yildiz und Gedik (2001) den positiven Zusammenhang zwischen Hyperandrogenämie und Insulinresistenz (unter anderem mit Nüchterninsulin, HOMA-IR) bei PCOS-Patientinnen zeigen. Patientinnen ohne Hyperandrogenämie waren hierbei weniger insulinresistent und lassen die Vermutung $\mathrm{zu}$, dass Hyperandrogenämie eine Insulinresistenz bedingen kann. In den Untersuchungen von Luque-Ramirez et al. (2010) wurde die Frage nach verschiedenen Mechanismen der Insulinresistenz aufgeworfen. Letztere sei - neben den wichtigen Faktoren Alter und BMI - bei PCOS-Patientinnen wesentlich mit Hyperandrogenämie (als gemessenes freies Testosteron), chronischer Entzündung und familiärer DiabetesDisposition assoziiert, wohingegen die Insulinresistenz der Patientinnen ohne Hyperandrogenämie andere Ursachen habe. 
Die Ergebnisse der Korrelationen zwischen Insulinresistenz und Hyperandrogenämie in Form eines erhöhten FAl entsprechen insofern der Studienlage, als dass in dieser Arbeit ebenfalls ein statistisch signifikanter Zusammenhang bestätigt wurde, welcher dennoch in beiden Gruppen unterschiedlich ausfiel. Interessant ist hierbei die Tatsache, dass der HOMA-IR lediglich bei PCOS-Patientinnen positiv mit dem FAI korreliert, wenn zugleich die AUC von Insulin in beiden Gruppen korreliert. Interessanterweise war in einer nachträglichen Auswertung die Korrelation von BMI mit FAI in beiden Gruppen statistisch nicht signifikant, wodurch in Anlehnung an Acien et al. (1999) die These, dass der bei PCOS-Patientinnen höhere BMI ursächlich für die unterschiedlichen Korrelationen ist, nicht bestätigt werden kann. Die ebenfalls im Nachhinein ausgewertete Korrelation von Alter mit FAl ergab in beiden Gruppen einen statistisch signifikanten Zusammenhang im Sinne einer negativen Korrelation, so dass auch dies keinen Erklärungsansatz darstellt. Der HOMA-IR bildet - auch im Kontext der in den Abschnitten 1.4.5 (S. 9), 4.2 .7 (s.u.) und 4.3 (S. 69 ff.) diskutierten Problematik der diagnostischen Wertigkeit des HOMAIR - die Insulinresistenz nicht vollends ab, so dass er bei den ausgewerteten Patientinnen ohne PCOS kein geeigneter Parameter zur Korrelation war. Die PCOSPatientinnen dieser Studie hatten wie gezeigt ein deutlich höheres Nüchterninsulin als Patientinnen ohne PCOS. Dagegen repräsentiert die Fläche unter der Kurve das Integral der Insulingesamtausschüttung, welches bei hoher Insulinsekretion in der Folge auch bei Patientinnen ohne PCOS zu einer Hyperandrogenämie führt.

\subsubsection{OGTT bei PCOS-Patientinnen und bei Patientinnen ohne PCOS}

PCOS-Patientinnen hatten zu allen drei gemessenen Zeitpunkten im OGTT höhere Insulinwerte und in der Folge auch ein höheres AUC Insulin als Patientinnen mit ausgeschlossenem PCOS. Ähnlich gestaltete sich der Unterschied des C-Peptids, dessen Werte nüchtern und nach einer Stunde bei Patientinnen mit PCOS höher waren. Die Indizes der Insulinresistenz HOMA und QUICKI waren in beiden Gruppen signifikant unterschiedlich; PCOS-Patientinnen waren entsprechend beider Indizes insulinresistent. Des Weiteren hatten PCOS-Patientinnen, die keinen Ovulationshemmer einnahmen, einen signifikant höheren HOMA-IR; dies konnte bei Patientinnen mit ausgeschlossenem PCOS nicht beobachtet werden. 
Hahn et al. (2004) beobachteten ebenfalls ein höheres AUC Insulin bei PCOSPatientinnen im Vergleich zu gesunden Kontrollpatientinnen. In der Studie von Tan et al. (2007) fehlt der Vergleich mit Kontrollpatientinnen im OGTT; man erkennt jedoch den positiven Zusammenhang von BMI und AUC Insulin. Luque-Ramirez et al. (2010) zeigten ein signifikant höheres AUC Insulin im 2-Stunden-OGTT bei PCOSPatientinnen.

Die Recherche ergab nur wenige vergleichbare Studien zur Unterscheidung von PCOS- und Kontrollpatientinnen anhand einer Insulinresistenz. Obgleich der Unterschied beider Gruppen in Parametern der Insulinresistenz evident ist, bleibt ein Vergleich mit Hahn et al. (2004) und Luque-Ramirez et al. (2010) problematisch, da in beiden Studien keine hyperandrogenen Kontrollpatientinnen untersucht wurden. In der vorliegenden Studie bestand die Kontrollgruppe nicht aus randomisierten, gesunden Patientinnen, sondern aus Patientinnen der Routineversorgung im Praxisalltag. Diese Patientinnen mit ausgeschlossenem PCOS hatten in dieser Studie dennoch per se eine Hyperandrogenämie (siehe oben), was als ein graduelles PCOS bewertet werden kann.

Wie eingangs beschrieben bestehen Kontroversen bezüglich diagnostischer Standards der Insulinresistenz, unter anderem auch auf Grund mangelnder allgemeingültiger Definition der Insulinresistenz (Azziz et al. 2009; Traub 2011). Für die klinische Praxis stellt sich die Frage, welcher Test in welcher Situation adäquat ist. Dabei kann zwischen dem Screening der Insulinresistenz bei PCOS-Patientinnen - im Vergleich zu Patientinnen mit ausgeschlossenem PCOS - und den Verlaufskontrollen bei negativem Befund oder auch bei Therapie mit Metformin unterschieden werden (siehe Abschnitt 4.4). Im Folgenden werden die Optionen des oralen Glukosetoleranztests (OGTT) und des HOMA-IR als Methode mit Nüchternwerten diskutiert. Die sogenannte Clamp-Technik, welche Wallace et al. (2004) als Goldstandard der direkten Verfahren zur Bestimmung einer Insulinresistenz beschreiben, wird auf Grund fehlender Praxistauglichkeit nicht berücksichtigt. Der HOMA-IR ist laut Wallace et al. (2004) ein probates Diagnostikum für die klinische Praxis. Azziz et al. (2009) betrachteten den HOMA-IR als nützlich, insbesondere in breiten epidemiologischen Studien - so wie in den Untersuchungen von Carmina und Lobo (2004) und DeUgarte et al. (2005). In beiden Studien wurden 
vorwiegend übergewichtige und adipöse PCOS-Patientinnen untersucht. Dagegen überprüften Fulghesu et al. (2006) die diagnostische Wertigkeit des HOMA-IR in einem Kollektiv normalgewichtiger Patientinnen, bei welchen der HOMA-IR in einer Vielzahl der Fälle fälschlicherweise keine Insulinresistenz erkannte. Diese Patientinnen zeigten eine erst im OGTT sichtbare Hyperinsulinämie, so dass der HOMA-IR mit einer niedrigen Sensitivität von $41 \%$ nicht zum Ausschluss früher metabolischer Störungen (Hyperinsulinämie) bei jungen, normalgewichtigen Patientinnen tauge. Fulghesu et al. (2006) empfehlen daher für dieses Patientenkollektiv ein 3-Stunden-OGTT. In einer systematischen Übersichtsarbeit der Androgen Excess Society analysierten Salley et al. (2007) die optimalen Methoden zum Screening einer gestörten Glukosetoleranz bei PCOS-Patientinnen. Demnach wird allen PCOS-Patientinnen bei Erstdiagnostik und im Falle normaler Glukosetoleranz alle zwei Jahre ein 2-Stunden-OGTT empfohlen: Patientinnen mit gestörter Glukosetoleranz sollten jährlich im Hinblick auf die Entwicklung eines Diabetes mellitus gescreent werden. Im Kontext derzeit uneindeutiger Evidenz zur gestörten Glukosetoleranz bei dünnen PCOS-Patientinnen wird vereinzelt empfohlen, lediglich adipöse PCOS-Patientinnen mittels OGTT zu screenen. Ein 2Stunden-OGTT wird somit für die Bewertung der Glukosetoleranz und Hyperinsulinämie derzeit als probatestes Mittel in der Praxis angesehen (Azziz et al. 2009). Darüber hinaus existieren gegenwärtig keine einheitlichen Kriterien, die den Grad der Hyperinsulinämie als Marker der Insulinresistenz im OGTT definieren. Insulinwerte von 80 bis $100 \mu \mathrm{U} / \mathrm{ml}$ im 2-Stunden-OGTT bedeuten Hyperinsulinämie; Werte über $300 \mu \mathrm{U} / \mathrm{ml}$ als Ausdruck schwerer Hyperinsulinämie implizieren indirekt eine Insulinresistenz (Azziz et al. 2009).

Das in dieser Arbeit untersuchte Kollektiv konnte sowohl mittels HOMA-IR als auch im 2-Stunden-OGTT anhand einer möglichen Insulinresistenz in PCOS-Patientinnen und solche mit ausgeschlossenem PCOS unterschieden werden. Der OGTT zeigte anhand der oben genannten absoluten Insulinwerte keine schwere, aber eine konsistente Hyperinsulinämie bei PCOS-Patientinnen. In der Literatur konnte keine Aussage zur Bewertung des Insulinwertes nach zwei Stunden gefunden werden. In dieser Arbeit war jeweils ein Abfall nach zwei Stunden zu beobachten. Ob einem Abfall eine positive Bedeutung beizumessen ist, bleibt ungeklärt und wäre ein interessanter Gegenstand zukünftiger Studien. Die diagnostische Aussagekraft des 
HOMA-IR kann bei den untersuchten Patientinnen vermutlich als hoch eingeschätzt werden, da diese im Kontrast zur Studie von Fulghesu et al. (2006) etwa zehn Jahre älter und einen BMI im adipösen Bereich hatten. Eine grundlegend andere Auswertung bzw. ein Studiendesign mit nach Alter und BMl differenzierten Patientinnen hätte diese These bestätigen können.

Dessen ungeachtet scheint in der Zusammenschau oben genannter Literatur, ein 2Stunden-OGTT bei Erstuntersuchung im Rahmen der PCOS-Diagnostik unabhängig vom BMI der Patientinnen sinnvoll zu sein. Daran anschließend bedarf es jedoch der Diskussion, welches diagnostische Mittel im Rahmen der Verlaufskontrolle unter der Therapie mit Metformin sinnvoll ist (siehe unten).

\subsection{Therapie mit Metformin bei 30 PCOS-Patientinnen}

Die Gruppe PCOS-Met mit 30 PCOS-Patientinnen hatte unter der Therapie mit Metformin innerhalb von sechs bis zwölf Monaten einen zweiten OGTT und ist daher für die Beurteilung der Metformintherapie bei PCOS-Patientinnen mit Insulinresistenz geeignet. Wie in Abschnitt 1.5.4 dargestellt wurde, wird nicht nur die Evidenz zur Verbesserung der Insulinresistenz, sondern auch die Verbesserung anderer Parameter unter der Therapie mit Metformin unterschiedlich bewertet. Gründe hierfür sind zum einen unterschiedliche Diagnostik und Definition der Insulinresistenz, und auf der anderen Seite das betrachtete Patientengut sowie die Dauer und Dosis der Behandlung mit Metformin.

\subsubsection{BMI}

Zum zweiten Untersuchungszeitpunkt konnte eine signifikante Gewichtsabnahme mit durchschnittlich 4,0 kg festgestellt werden. Der BMI sank durchschnittlich um 1,5 auf rund $32 \mathrm{~kg} / \mathrm{m}^{2}$. Dabei bestand kein statistisch relevanter Einfluss eines Ovulationshemmers.

Signifikante BMI-Senkungen um einen Punkt und signifikante Gewichtsabnahmen um 2,8 kg fanden auch Tang et al. (2006) bei adipösen PCOS-Patientinnen (BMI $\geq 30$ $\left.\mathrm{kg} / \mathrm{m}^{2}\right)$ unter einer sechsmonatigen Therapie mit Metformin $(2 \times 850 \mathrm{mg} / \mathrm{d})$. Allerdings konnte im Vergleich zu einer Placebogruppe kein signifikanter Unterschied des 
Gewichts und BMI festgestellt werden, so dass der Einfluss von Metformin auf diese Parameter als gering bewertet wurde. In den Untersuchungen von Bridger et al. (2006) mit einer kurzzeitigen dreimonatigen Metformintherapie (2 x $750 \mathrm{mg} / \mathrm{d}$ ) zeigte sich keine signifikante Abnahme des BMI bei allerdings nur 22 Jugendlichen im Alter von 13 bis 18 Jahren. Dagegen fanden Arslanian et al. (2002) bei adipösen Jugendlichen eine signifikante BMI-Abnahme. Otta et al. (2009) fanden bei 30 PCOS-Patientinnen keine signifikante BMI-Abnahme unter viermonatiger Metformintherapie $(1500 \mathrm{mg} / \mathrm{d}$ ) mit gleichzeitiger Lebensstiländerung, wohl aber eine signifikante Abnahme des Bauchumfangs und des Taille-Hüft-Verhältnisses - auch im Vergleich zu einer Gruppe mit Diät und Sport. Auch in der Studie von Oppelt et al. (2010) zu einer zweijährigen Langzeitbehandlung mit Metformin (2 x 850 mg/d) ergab sich keine signifikante Änderung des BMI bei 26 adipösen PCOS-Patientinnen. Dagegen sank bei einem deutschen Kollektiv der BMI signifikant von anfangs 32 $\mathrm{kg} / \mathrm{m}^{2}$ auf 29,0 und 28,3 kg/m² nach sechs und zwölf Monaten; die Therapie mit Metformin war dabei gewichtsadaptiert (Hahn et al. 2004).

Der Trend aller oben aufgeführten Studien, dass bisher keine Evidenz für eine Verbesserung des BMI unter der Therapie mit Metformin existiert, konnte in einer aktuellen Analyse der Cochrane Collaboration bestätigt werden (Tang et al. 2010). Die vorliegende Studie ähnelt der Studie von Hahn et al. (2004) hinsichtlich Dauer der Studie, Alter, BMI und Anzahl der PCOS-Patientinnen, obgleich der BMI eine große Spannweite aufwies und somit in der vorliegenden Studie ein eher heterogenes Kollektiv betrachtet wurde. Die längere Dauer beider Studien lässt die These zu, dass offenkundig eine kurzzeitige Metformintherapie von drei bis sechs Monaten nicht geeignet ist, um den BMI günstig zu beeinflussen. Da aus praxisorganisatorischen Gründen ein Zeitraum von sechs bis zwölf Monaten für den zweiten OGTT mit in die Auswertung gebracht wurde, bewegt sich diese Studie zwischen Kurz- und Langzeittherapie mit Metformin. Schließlich könnte auch der Einfluss von diätetischen Maßnahmen und Sport während der Metformintherapie eine Rolle für das signifikante Ergebnis darstellen. Beides wurde auf Grund mangelnder Informationen bei der Datenerhebung nicht berücksichtigt, obgleich alle PCOS-Patientinnen im Endokrinologikum über den Nutzen von Allgemeinmaßnahmen in der Therapie des PCOS aufgeklärt wurden. 


\subsubsection{Akne, Hirsutismus und Zyklusstörungen}

Für die Auswertung dieser Parameter wurden lediglich Patientinnen ohne Ovulationshemmer berücksichtigt, da der Einfluss von Ovulationshemmern statistisch relevant war und die Ergebnisse durch diese Störgröße verzerrt worden wären.

Unter den klinischen PCOS-Parametern hatte sich das Auftreten von Akne und Hirsutismus nicht signifikant geändert, obgleich ein tendenzieller $(p<0,068)$ Rückgang des Hirsutismus beobachtet wurde. Dagegen konnte eine signifikante Reduktion der Zyklusstörungen um 36,84 \% zum zweiten Untersuchungszeitpunkt festgestellt werden.

Die Untersuchungen von Tan et al. (2007) zur sechsmonatigen (gewichtsadaptierten) Metformintherapie zeigten signifikante Verbesserungen von Akne, Hirsutismus und Zyklusstörungen sowohl bei übergewichtigen und adipösen, als auch bei normalgewichtigen PCOS-Patientinnen. In der Studie von Hahn et al. (2004) mit gewichtsadaptierter Metformindosis besserten sich alle drei Parameter ebenfalls mit zunehmender Behandlungsdauer. Oppelt et al. (2010) beobachteten bei adipösen PCOS-Patientinnen während ihrer zweijährigen Langzeittherapie mit Metformin $(2 \mathrm{x}$ $850 \mathrm{mg} / \mathrm{d}$ ) eine signifikante Besserung des Hirsutismus im Zuge verbesserter Hyperandrogenämie. Keinen Effekt auf das Auftreten von Akne und Hirsutismus zeigte eine viermonatige Metformintherapie (1500 mg/d) in Kombination mit Lebensstiländerung bei 30 PCOS-Patientinnen. Dabei besserten sich die Zyklusstörungen signifikant sowohl unter Metformin als auch unter Placebo (Otta et al. 2009). Zum gleichen Ergebnis kamen Tang et al. (2006) bei adipösen PCOSPatientinnen $\left(\mathrm{BMl} \geq 30 \mathrm{~kg} / \mathrm{m}^{2}\right)$, wo sich die Zyklusstörungen sowohl unter einer sechsmonatigen Therapie mit Metformin $(2 \times 850 \mathrm{mg} / \mathrm{d})$ als auch unter diätetischen Maßnahmen besserten; zwischen beiden Gruppen bestand kein signifikanter Unterschied. Einer Übersicht der Cochrane Collaboration ist zu entnehmen, dass Metformin auf indirektem Wege durch Ovulationsinduktion den Zyklus normalisieren kann (Tang et al. 2010). Die Evidenz zum Einfluss von Metformin auf Hirsutismus und Akne ist derzeit noch unklar (Badawy und Elnashar 2011; Lowenstein 2006). Eine interessante aktuelle Studie zeigte, dass eine frühzeitige Metformintherapie bei Kindern, die eine Prädisposition für die Entwicklung eines PCOS besitzen, die Entstehung von Hirsutismus verhindern oder zumindest hinauszögern kann (Ibanez et al. 2011). 
Die Studienlage zum Einfluss von Metformin auf klinische PCOS-Parameter ist somit nicht eindeutig und je nach Parameter differenziert zu bewerten. Die Patientinnen der vorliegenden Arbeit profitierten klinisch hinsichtlich verbesserter Zyklusstörungen von einer Therapie mit Metformin, vergleichbar mit den Studien von Tan et al. (2007), Otta et al. (2009) und Tang et al. (2006). Der Effekt von Metformin allein kann anhand der ausgewerteten Daten nicht sicher beurteilt werden, da etwaige Einflussgrößen wie BMI und andere gewichtsreduzierende Maßnahmen für die Auswertung nicht berücksichtigt wurden. Ein wesentliches Manko ist zudem die fehlende Kontrollgruppe mit gesunden Frauen oder PCOS-Patientinnen mit Placebo statt Metformin, um validere Aussagen treffen zu können. Dessen ungeachtet kann durch das untersuchte Kollektiv die Hypothese aufgestellt werden, dass sich Zyklusstörungen im Zuge einer signifikanten Gewichtsreduktion bessern. Metformin könnte durch gesunkene Androgene (siehe unten) den Hirsutismus in dieser Studie tendenziell verbessert haben. Für die Bewertung von Akne und Hirsutismus bleiben die nicht standardmäßige Verwendung des Ferriman-Gallway-Score und der Einfluss anderer therapeutischer Mittel problematisch.

\subsubsection{FAl und SHBG}

Beide Parameter waren statistisch relevant von der Einnahme eines Ovulationshemmers beeinflusst. Der freie Androgenindex sank allein unter Therapie mit Metformin im Mittel um 1,8 auf 9,77 Punkte ( \pm 9,05 SD; Median: 7,80), was jedoch keinen signifikanten Abfall im Vergleich zur Erstuntersuchung darstellte $(p=0,3324)$. Das SHBG blieb unter Metformintherapie statistisch unverändert auf einem niedrignormalen Niveau. Bei Auswertung aller 30 Patientinnen - inklusive derer mit Ovulationshemmer - sank der FAl signifikant im Vergleich zur Erstuntersuchung. Der Einfluss von Ovulationshemmern wird hierbei offenkundig, obwohl in beiden Fällen der FAI weit über dem für Frauen geltenden Normwert von $\leq 3,5$ lag. Bei Auswertung aller 30 Patientinnen ergab sich trotz Ovulationshemmer kein signifikanter Anstieg des SHBG bei insgesamt jedoch höheren SHBG-Spiegeln. Tang et al. (2006) beobachteten bei 56 adipösen PCOS-Patientinnen einen signifikanten Abfall des FAI unter sechsmonatiger Metformintherapie $(2 \times 850 \mathrm{mg} / \mathrm{d})$, hauptsächlich dem Abfall des Gesamttestosteron geschuldet. Sie vermuteten auf 
Grund unveränderter Insulinsensitivität und keinem signifikantem Anstieg des SHBG einen direkten Effekt von Metformin auf die ovarielle Androgenproduktion. Einen ähnlichen Effekt allerdings mit gebesserter Insulinresistenz konnte unter kurzzeitiger Therapie mit Metformin festgestellt werden (Hahn et al. 2004; Otta et al. 2009; Tan et al. 2007). Bei adipösen, jugendlichen PCOS-Patientinnen erreichte man unter dreimonatiger Metformintherapie signifikante Abnahmen der Androgene bei verbesserter Insulinresistenz (Arslanian et al. 2002; Bridger et al. 2006). Schließlich wurde ein hochsignifikant fallender FAI auch bei 24-monatiger Langzeittherapie mit Metformin beobachtet (Oppelt et al. 2010). In dieser Studie wurde ein statistisch relevanter Anstieg des SHBG nach zwei Jahren beobachtet. Dagegen konnte in der Studie von Arslanian et al. (2002) und in einer aktuellen Metanalyse von Tang et al. (2010) ein solcher signifikanter Anstieg des SHBG unter der Therapie mit Metformin nicht beobachtet werden.

In der vorliegenden Arbeit hatte Metformin lediglich in Kombination mit einem Ovulationshemmer einen positiven Effekt auf den FAI. Ein Manko stellt wiederum die fehlende Kontrollgruppe zur Beurteilung dar. Obwohl sich der Mittelwert des FAI bei den PCOS-Patientinnen zur Zweituntersuchung weiterhin nicht im Normbereich bewegte, lag er dennoch im Werteniveau der oben genannten Studien. Dagegen entspricht die vorliegende Arbeit der aktuellen Studienlage, was den fehlenden Effekt von Metformin auf das SHBG anbelangt.

So bleibt derzeit die Frage ungeklärt, inwieweit Metformin im Zuge verbesserter Insulinresistenz (HOMA-IR, siehe S. 74 ff.) zur absoluten Reduktion einer Hyperandrogenämie nützt. Da Hyperandrogenämie nach heutigem Verständnis als ein unabdingbares Kriterium des PCOS angesehen wird, sollte daher der Fokus zur Bewertung von Metformin auf der klinischen Bedeutung (Hirsutismus, Zyklusstörungen etc.) liegen. Diese Studie konnte demonstrieren, dass PCOSPatientinnen klinisch mit normalisiertem Menstruationszyklus von der Therapie mit Metformin - und allgemeiner Gewichtsabnahme - profitieren.

\subsubsection{OGTT und Insulinresistenz}

Der Vergleich beider OGTT muss für die verschiedenen Parameter differenziert betrachtet werden. Die Nüchternglukose sank signifikant zum zweiten OGTT, 
wohingegen die Werte nach einer Stunde signifikant bzw. nach zwei Stunden tendenziell anstiegen. Ein signifikanter Abfall wurde ebenfalls beim Nüchterninsulin festgestellt. Alle restlichen Werte veränderten sich nicht signifikant.

Die aus den Werten des OGTT berechnete Fläche unter der Kurve (AUC) änderte sich weder für Glukose, noch für Insulin und C-Peptid signifikant. Ein tendenzieller Anstieg $(p<0,1)$ wurde für die AUC von Glukose berechnet. Die beiden Indizes der Insulinresistenz HOMA-IR und QUICKI hatten sich zum zweiten Untersuchungszeitpunkt signifikant im Sinne einer verbesserten Insulinresistenz geändert. Der HOMA-IR sank zwar fast um einen Punkt auf 2,9 Punkte, lag jedoch weiterhin im Bereich einer wahrscheinlichen Insulinresistenz.

Die Evidenz zur Verbesserung der Insulinresistenz unter der Therapie mit Metformin wird gegenwärtig kontrovers diskutiert, da eine Bewertung durch große Heterogenität der Definitionen einer Insulinresistenz, der betrachteten Parameter und des Patientenkollektivs erschwert wird (Traub 2011). Die Übersicht der Cochrane Collaboration zeigte eine Reduktion des Nüchterninsulins nicht bei adipösen, sondern nur bei normalgewichtigen PCOS-Patientinnen (Tang et al. 2010). In den Untersuchungen von Tan et al. (2007) verbesserte Metformin nach sechs Monaten die Parameter HOMA-IR, Nüchterninsulin und AUC Insulin bei adipösen Patientinnen, wobei der HOMA-IR und das Nüchterninsulin bei normalgewichtigen, nicht wegen Insulinresistenz vorbehandelten Patientinnen ebenfalls signifikant abnahmen. Oppelt et al. (2010) beobachteten bei adipösen Patientinnen nach zweijähriger Therapie mit Metformin $(2 \times 850 \mathrm{mg} / \mathrm{d}$ ) lediglich Abnahmen der Insulinwerte nüchtern und nach zwei Stunden, der gesunkene HOMA-IR erreichte keine Signifikanz. Hahn et al. (2004) zeigten bei 30 adipösen PCOS-Patientinnen im Verlauf von sechs und zwölf Monaten eine stetig verbesserte Insulinresistenz mit gesunkenem HOMA-IR, verbesserter Hyperinsulinämie mit Abnahme des AUC Insulin sowie eine Abnahme des Glukosewertes nach zwei Stunden. Bei jugendlichen, adipösen Patientinnen verbesserte sich nach dreimonatiger Therapie die orale Glukosetoleranz; AUC Glukose, AUC Insulin und Nüchterninsulin sanken signifikant (Arslanian et al. 2002). Dagegen erreichten verbesserte AUC Insulin und HOMA-IR in der Studie von Bridger et al. (2006) bei ebenfalls dreimonatiger Metformintherapie jugendlicher Patientinnen keine Signifikanz. Metformin (1500 $\mathrm{mg} / \mathrm{d}$ ) für vier Monate in Kombination mit einer Änderung des Lebensstils bewirkte 
signifikante Abnahmen des HOMA-IR und Nüchterninsulins bei normalgewichtigen Patientinnen (Otta et al. 2009). Im Einklang mit der Cochrane Collaboration zeigte eine frühere Studie von Tang et al. (2006) bei stark adipösen PCOS-Patientinnen keine Änderungen des Nüchterninsulins und auch der Insulinsensitivität (QUICKI) unter sechsmonatiger Therapie mit 2 × $850 \mathrm{mg}$ Metformin.

Im Gegensatz zur letztgenannten Studie und der Analyse der Cochrane Collaboration konnte in der vorliegenden Studie sehr wohl eine Verbesserung des Nüchterninsulins bei adipösen PCOS-Patientinnen demonstriert werden. Im Zusammenhang mit verbesserter Nüchternglukose erklärt dies den signifikanten Abfall des HOMA-IR. Darüber hinaus sind auf dem Fundament eines sinkenden Nüchterninsulins sowohl erhöhte Glukosewerte nach einer und zwei Stunden als auch der tendenzielle Anstieg von AUC Glukose logisch zu interpretieren. Die Hyperinsulinämie (AUC Insulin) blieb unbeeinflusst. In Bezug zur Cochrane Collaboration und der früheren Untersuchung von Tang et al. (2006) könnte das heterogene Kollektiv der vorliegenden Studie mit großer Spannweite im BMI und Alter das unerwartet gute Ergebnis erklären. Eine Differenzierung der Patientinnen anhand des BMI - wie in den Untersuchungen von Tan et al. (2007) demonstriert wurde - hätte an dieser Stelle möglicherweise zu neuen Erkenntnissen geführt. Demungeachtet wäre dies in dieser Arbeit mit der zu kleinen Stichprobe $(n=30$; davon allein 22 mit $B M I \geq 30 \mathrm{~kg} / \mathrm{m}^{2}$ ) nicht realisierbar gewesen. Eine Differenzierung anhand des BMI kann aber der besseren Einordnung des diagnostischen Nutzens des HOMA-IR dienen, welcher nach Fulghesu et al. (2006) bei jungen, nicht adipösen PCOS-Patientinnen nur eine geringe Sensitivität (41\%) aufweist. Die Studie von Fulghesu et al. (2006) offenbart zudem, dass ein OGTT über drei Stunden eine höhere diagnostische Wertigkeit in diesem speziellen Patientenkollektiv impliziert, was bei Anwendung in der vorliegende Studie zu abweichenden Ergebnisse der berechneten Flächen unter Kurve hätte führen können. Diese Hypothese lässt sich insofern stützen, als dass Bridger et al. (2006) bei jungen, aber adipösen Patientinnen kein signifikant verändertes AUC Insulin bei einem 2-Stunden-OGTT beobachteten, wohingegen in anderen Studien mit einem OGTT über drei Stunden dies dennoch erreicht wurde (Arslanian et al. 2002; Hahn et al. 2004; Tan et al. 2007). 
Zusammenfassend ergaben sich in der vorliegenden Studie zur sechs- bis zwölfmonatigen Metformintherapie bei 30 adipösen PCOS-Patientinnen folgende signifikante Änderungen: Gewicht und BMI ( $\downarrow)$, Zyklusstörungen ( $\downarrow)$, Insulin nüchtern $(\downarrow), H O M A-I R(\downarrow)$, QUICKI ( $\uparrow$, im Sinne einer verbesserten Insulinsensitivität bzw. abnehmenden Insulinresistenz), Glukose nüchtern ( $\downarrow)$, Glukose nach einer Stunde $(\uparrow)$. Tendenzen $(p<0,1)$ erreichten die Parameter Hirsutismus $(\downarrow)$ und Glukose nach zwei Stunden ( $\uparrow$ ).

Im Hinblick auf die aufgeworfene Frage nach dem adäquaten diagnostischen Mittel zur Erfassung der Insulinresistenz hat sich in der vorliegenden Studie der HOMA-IR besser zur Verlaufskontrolle der Metformintherapie geeignet als ein 2-StundenOGTT. Wie bereits des Öfteren erwähnt, hätte eine Differenzierung der Patientinnen anhand des BMI und des Alters mehr Informationen geboten, wobei die Patientinnen in Abgrenzung zu Fulghesu et al. (2006) auch nach BMI-Bereinigung immer noch etwa 10 Jahre älter wären. Weiterhin wäre ein OGTT über drei Stunden in Betracht zu ziehen. Dagegen spricht - unabhängig von Gründen problematischer Durchführung aus abrechnungbedingten Aspekten - einerseits die Tatsache, dass der 2-Stunden-Glukosewert das wichtigste Kriterium einer pathologischen Glukosetoleranz darstellt (Balkau 2000). Zum anderen besitzt ein 2-Stunden-OGTT bei Nichtdiabetikern die gleiche diagnostische Wertigkeit wie zeitlich längere OGTT (Dalla Man et al. 2005). Daher bleibt ein OGTT über drei Stunden den Studien vorbehalten und eignet sich weniger für die Praxis. Unklar bleibt auch die Bewertung der Insulinkurve im OGTT, wie beispielsweise ein Abfall nach zwei Stunden zu interpretieren ist. Es bedarf somit einer entsprechenden Empfehlung zur Insulinresistenz und ihrer Diagnostik beim PCOS, wie sie von Salley et al. (2007) im Namen der Androgen Excess Society für die Glukosetoleranz ausgesprochen wurde. 


\subsection{Kritik und Ausblick}

Rückblickend ergeben sich im Lichte der diskutierten Ergebnisse einige kritische Anmerkungen zur vorliegenden Studie. Darüber hinaus entstanden interessante Fragestellungen für zukünftige Arbeiten.

Bei der Datenerhebung erwies es sich als problematisch, dass - bedingt durch zum Teil unterschiedliche Dokumentation des Ärzteteams im Endokrinologikum - die Patientinnen nicht stets nach einem standardisierten Vorgehen diagnostiziert wurden und in der Folge nicht bei allen Patientinnen alle Parameter ausgewertet werden konnten. Für die Auswertung zukünftiger Studien wäre es hilfreich, die folgenden Punkte zu verbessern:

Die Diagnose des PCOS anhand der Rotterdam-Kriterien wird zwar noch in einigen Studien angewandt, sollte jedoch in Zukunft besser durch die aktuelle Definition der AES (Azziz et. Al 2006) ersetzt werden. Nach diesen Kriterien hätte möglicherweise bei einigen Patientinnen der Gruppe 5 mit einem nach Rotterdam-Kriterien ausgeschlossenen PCOS dennoch ein PCOS diagnostiziert werden können. Der $\mathrm{LH} / \mathrm{FSH}$-Quotient sollte nicht bloß einmalig bei Diagnosestellung des PCOS erhoben werden. Bei Blutentnahme sollte zur besseren Interpretation der Laborwerte der Zyklustag der Patientin vermerkt werden - optimal wäre der dritte bis fünfte Zyklustag. Da sich die vorliegende Arbeit nicht explizit mit dem Metabolischen Syndrom beschäftigte, wäre dies für zukünftige Arbeiten ein interessanter Aspekt und bedarf in der Folge der Erhebung zusätzlicher Untersuchungen wie beispielsweise des sogenannten waist-hip-ratio (Verhältnis von Taille zu Hüfte) oder allgemein des Bauchumfangs. Zur besseren Vergleichbarkeit sollte ein Hirsutimus bei allen Patientinnen mit Hilfe des Ferriman-Gallway-Score evaluiert werden. Vitamin-DMangel sollte vor dem Hintergrund aktueller Studien bei Laborwerterhebung mit berücksichtigt werden. Dies gilt in gleicher Weise für die Schilddrüsendiagnostik im Hinblick auf eine Hashimoto-Thyreoiditis. Weiterhin sollte zukünftig bei allen PCOSPatientinnen das CRP bestimmt werden. Der Einfluss von verschiedenen Ovulationshemmern auf die Therapie und den Verlauf des PCOS sollte stets berücksichtigt werden.

Eine Auswertung, nach Alter und BMI differenziert, ist für zukünftige Studien zu empfehlen. Rückblickend betrachtet wäre die ursprüngliche Gruppe PCOS $\varnothing$ Met (4) 
als Kontrollgruppe für die Evualuation der Metformintherapie geeignet gewesen, wenn sämtliche Parameter nicht bloß zu einem Untersuchungszeitpunkt erhoben worden wären. Ebenfalls wäre eine Kontrollgruppe mit gesunden Frauen zum Vergleich der Therapie mit Metformin oder zur besseren Bewertung diagnostischer Tests der Insulinresistenz empfehlenswert. Letzteres eröffnet zugleich die Frage nach der Realisierbarkeit und Praxistauglichkeit im Rahmen der Routineversorgung. Somit können anhand der Ergebnisse dieser Arbeit Empfehlungen zur Diagnostik der Insulinresistenz nur mit Einschränkung abgegeben werden.

Dessen ungeachtet und in der Zusammenschau der Literatur könnte ein Fazit für die Praxis sein, dass es für die Diagnostik der Insulinresistenz oder allgemeiner Hyperinsulinämie junger, normalgewichtiger PCOS-Patientinnen eines 2-StundenOGTT bedarf. Im Falle älterer, übergewichtigerer Patientinnen könnte der HOMA-IR diagnostisch genügen. Weitere Studien mit nach Alter und BMI differenzierten Patientinnen sind notwendig, um diese These zu verifizieren. 


\section{Zusammenfassung}

Das Polyzystische Ovarsyndrom (PCOS) betrifft circa sechs bis zehn Prozent aller Frauen im gebärfähigen Alter und ist eine der häufigsten endokrinologischen Erkrankungen in dieser Lebensphase. Neben der vielfältigen klinischen Manifestation einer Hyperandrogenämie rücken metabolische Störungen mit Insulinresistenz und kardiovaskulären Folgeerkrankungen in den Fokus von Klinik und Forschung des PCOS. Das Medikament Metformin gilt als etablierte Therapieoption beim PCOS, wenngleich der Therapieerfolg je nach klinischer Indikation durchaus unterschiedlich ausfällt. Aus Mangel an evidenzbasierten Empfehlungen existiert derzeit eine Kontroverse, was die Diagnostik und klinische Bewertung einer Insulinresistenz beim PCOS anbelangt.

Die vorliegende Dissertation untersuchte retrospektiv die Routineversorgung von Patientinnen mit PCOS in einer Praxis für Endokrinologie. Es wurden die Diagnosesicherheit des PCOS unter Praxisbedingungen und der therapeutische Effekt von Metformin unter einer sechs- bis zwölfmonatigen Therapie analysiert. Zudem wurde zur kontroversen Diskussion eines geeigneten diagnostischen Mittels einer Insulinresistenz Stellung bezogen.

PCOS-Patientinnen unterschieden sich in vielen krankheitstypischen Parametern eindeutig von Patientinnen mit ausgeschlossenem PCOS, welche signifikant weniger an Hirsutismus und Zyklusstörungen litten sowie einen niedrigeren freien Androgenindex (FAl) und LH/FSH-Quotienten aufwiesen. PCOS-Patientinnen hatten ein höheres Nüchterninsulin, eine höhere Insulingesamtausschüttung und waren anhand der Indizes HOMA-IR und QUICKI insulinresistent. Die Parameter SHBG, Akne und Hashimoto-Thyreoiditis waren für die Unterscheidung beider Gruppen ungeeignet, da sie nicht signifikant unterschiedlich auftraten. Letztlich sind alle untersuchten Parameter vor dem Hintergrund zu bewerten, dass PCOS-Patientinnen mit durchschnittlich sieben Jahren erheblich jünger und in der Tendenz auch übergewichtiger als ihre Vergleichspatientinnen waren. Zudem litten alle untersuchten Patientinnen bei Betrachtung des FAI unter einer Hyperandrogenämie. Überdies kann die gewählte Gruppenzuordnung anhand der Rotterdam-Kriterien zur 
Definition eines PCOS im Nachhinein als problematisch angesehen werden, da laut der aktuellen Leitlinie zur Definition des PCOS (Androgen Excess Society (AES), Azziz et al. 2006) die Hyperandrogenämie das maßgebliche Charakteristikum in der Pathophysiologie des PCOS darstellt. Nach diesen Definitionskriterien wäre die Gruppenverteilung mutmaßlich unterschiedlich ausgefallen. Die Berücksichtigung dieser aktuellen Definition und die unter Abschnitt 4.4 (S. 77) geäußerten Punkte könnten zu einer Verbesserung der Diagnosesicherheit in der Routineversorgung von Patientinnen mit PCOS in der endokrinologisch-spezialisierten Praxis beitragen.

Im Vergleich zur diskutierten Literatur lag die vorliegende Studie mit sechs- bis zwölfmonatiger Metformintherapie im Bereich zwischen einer Kurz- und Langzeittherapie.

Bei derzeit noch fehlender Evidenz wurden unter der Therapie mit Metformin bei 30 adipösen PCOS-Patientinnen dennoch signifikante BMI-/ und Gewichtsabnahmen von durchschnittlich $1,5 \mathrm{~kg} / \mathrm{m}^{2}$ bzw. 4,0 kg Körpergewicht erzielt. Im Einklang mit vielen Studien konnte bei $36 \%$ der Patientinnen eine Normalisierung des Menstruationszyklus erreicht werden, was einer signifikanten Reduktion der Zyklusstörungen entsprach. Entgegen der aktuellen Studienlage wurde der FAI statistisch nicht relevant beeinflusst; lediglich in Kombination mit einem Ovulationshemmer sank der FAI signifikant, wobei weiterhin eine Hyperandrogenämie vorlag. Das SHBG stieg wie erwartet nicht an. Die Patientinnen waren unter Metformintherapie weniger insulinresistent mit signifikant niedrigerem Nüchterninsulin, niedrigerem HOMA-IR und höherem QUICKI (im Sinne einer abnehmenden Insulinresistenz). In diesem Kontext blieb die Frage offen, ob Metformin allein im Zuge verbesserter Insulinresistenz zur klinisch relevanten Reduktion einer Hyperandrogenämie beiträgt. Eine Vergleichsgruppe mit gesunden Frauen bzw. PCOS-Patientinnen mit Placebo statt Metformin, die Differenzierung anhand des BMI sowie die Berücksichtigung eines möglichen Einflusses von diätetischen Maßnahmen und Sport hätten letztlich zu einer valideren Bewertung des Effekts von Metformin und auch der Diagnostik der Insulinresistenz in dieser Studie geführt. Dennoch konnte in dieser Arbeit demonstriert werden, dass PCOS-Patientinnen klinisch im Sinne eines normalisierten Menstruationszyklus von der Therapie mit Metformin - und allgemeiner Gewichtsabnahme - profitieren. 
Zur Diagnostik einer Insulinresistenz waren HOMA-IR, QUICKI und ein OGTT über zwei Stunden geeignet, insulinresistente PCOS-Patientinnen von Patientinnen ohne PCOS und ohne Insulinresistenz zu unterscheiden. Die diagnostische Aussagekraft des HOMA-IR kann bei den untersuchten Patientinnen auf Grund des Alters und des $\mathrm{BMI}$ als hoch eingeschätzt werden. Zudem war der HOMA-IR zur Verlaufskontrolle der Metformintherapie besser geeignet als ein OGTT über zwei Stunden. Bei derzeit fehlender Literatur bleibt die Wertung des in beiden Abschnitten beobachteten Trends im Verlauf der Insulinkurve im OGTT mit einem Abfall nach zwei Stunden unbeantwortet. Weitere Untersuchungen mit den oben genannten Verbesserungen im Studiendesign sind bei aktuell fehlender Evidenz zur Diagnostik einer Insulinresistenz erforderlich. 


\section{Anhang}

\subsection{Abbildungsverzeichnis}

Abbildung 1: Auswirkungen der Insulinresistenz beim PCOS …...................................... 5

Abbildung 2: Polyzystisches Ovar im transvaginalen Ultraschall ...................................... 8

Abbildung 3: Therapieoptionen beim PCOS anhand verschiedener Symptome..................14

Abbildung 4: Varianzanalyse der Altersmittelwerte in den Gruppen 1 bis 5 ......................28

Abbildungen 5, 6 und 7: Darstellung des Körpergewichts anhand des BMI. ....................29

Abbildung 8: Varianzanalyse der BMI-Mittelwerte in den Gruppen 1 bis 5......................30

Abbildungen 9 und 10: Gruppen des ursprünglichen Gesamtkollektivs und nach

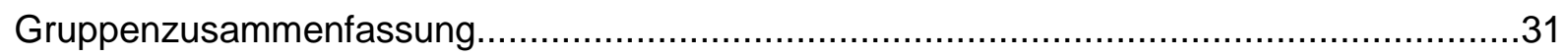

Abbildung 11: Darstellung der Mittelwerte des Alters in beiden Gruppen..........................32

Abbildungen 12 und 13: Verteilung von Hirsutismus und Zyklusstörungen in beiden

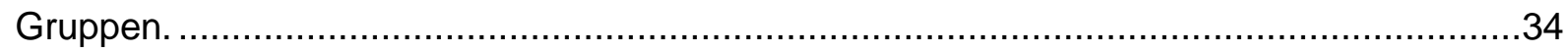

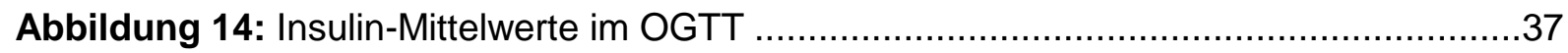

Abbildung 15: Zusammenhang zwischen BMI und LH/FSH-Quotient in beiden Gruppen ...41

Abbildung 16: Zusammenhang von AUC Insulin und SHBG in beiden Gruppen................42

Abbildung 17: Zusammenhang zwischen AUC Insulin und FAI in beiden Gruppen ...........43

Abbildung 18: Zusammenhang zwischen HOMA-IR und FAI in beiden Gruppen...............44

Abbildung 19: Gewichtsverteilung zu beiden Untersuchungszeitpunkten .........................46

Abbildung 20: Mittelwerte von Glukose in beiden OGTT...........................................49

Abbildung 21: Darstellung der Insulin-Mittelwerte in beiden OGTT .................................49

Abbildung 22: Mittelwert des HOMA-IR in beiden OGTT ...........................................

Abbildung 23: Mittelwert des FAl zu beiden Untersuchungszeitpunkten ..........................53

Abbildung 24: Einfluss von Ovulationshemmern und Hyperinsulinämie auf den FAI...........55 


\subsection{Tabellenverzeichnis}

Tabelle 1: Vergleich der Diagnose-Kriterien des PCOS............................................... 2

Tabelle 2: Gruppeneinteilung des Gesamtkollektivs. ..................................................19

Tabelle 3: Referenzwerte im OGTT für Vollblutglukose kapillär ........................................22

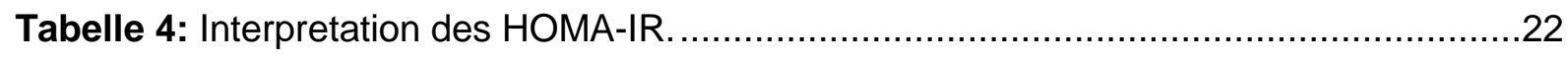

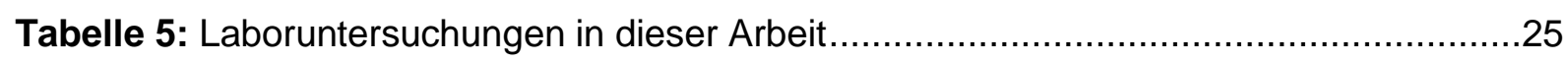

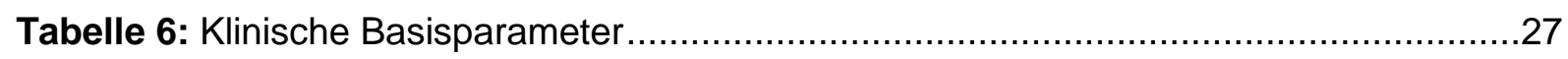

Tabelle 7: Klinische Basisparameter des neuen Gesamtkollektivs....................................31

Tabelle 8: Klinische Basisparameter der beiden Gruppen. ............................................32

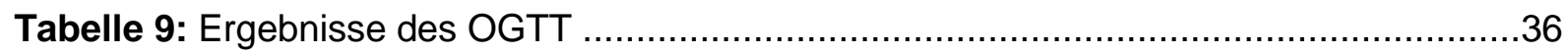

Tabelle 10: Fläche unter der Kurve von Glukose, Insulin und C-Peptid. .............................37

Tabelle 11: Einfluss des Ovulationshemmers auf Parameter von PCOS-Patientinnen ........39

Tabelle 12: Einfluss d. Ovulationshemmers auf Parameter von Patientinnen ohne PCOS...39

Tabelle 13: Gewicht und BMI zu beiden Untersuchungszeitpunkten .................................45

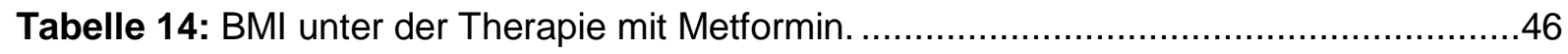

Tabelle 15: Auftreten von Zyklusstörungen zu beiden Untersuchungszeitpunkten ..............47

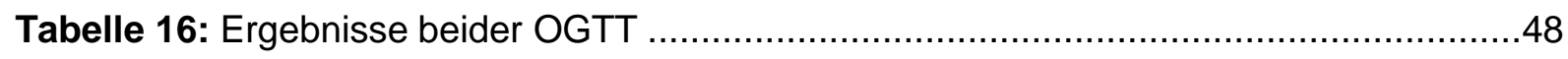

Tabelle 17: Fläche unter der Kurve für Glukose, Insulin und C-Peptid jeweils im ersten und zweiten OGTT. .50

Tabelle 18: Einfluss des Ovulationshemmers bei Erstuntersuchung .................................52

Tabelle 19: Einfluss des Ovulationshemmers bei der zweiten Untersuchung.......................52 


\subsection{Patienteninformation Metformin}

Metformin ist ein seit Jahrzehnten in der Behandlung der Blutzuckerkrankheit (Diabetes mellitus) zugelassenes Medikament. Es verbessert die Ansprechbarkeit des Gewebes für Insulin und führt somit zu einer verbesserten Blutzucker- und Stoffwechseleinstellung.

\section{Zusätzliche Wirkung von Metformin bei PCO}

- Abnahme der zuvor erhöhten männlichen Hormone und der vermehrten Körperbehaarung.

- Normalisierung der zuvor gestörten Zyklusblutung, das Eintreten einer Schwangerschaft ist möglich.

- Erleichtert das Abnehmen, da es den Appetit vermindert.

\section{Kontraindikationen}

- Extreme Fastenkuren ( mit weniger als 1000 Kalorien /die) wegen Gefahr der Laktatazidose

- Alkoholkonsum (evtl. kl. Menge möglich)

- 2 Tage vor und nach O.P. oder Verabreichung von intravenösen Röntgenkontrastmittel

- Schwangerschaft

- Schwere Leber- und Nierenfunktionsstörung

- Herz- und Lungenerkrankung

- Tumorleiden

\section{Nebenwirkungen}

- Störungen des Magen-Darm-Traktes: Metallischer Geschmack im Mund, leichte Übelkeit und Durchfall, selten Erbrechen oder Bauchkrämpfe

- Selten Anämie

- Sehr selten lebensgefährliche Laktatazidose mit Muskelkrämpfen, schweren Magen-DarmStörungen, Schwäche und ggf. Koma

\section{Dosierung}

\begin{tabular}{|l|l|l|l|}
\hline & Morgens & Mittags & Abends \\
\hline 1. Woche & 0 & 0 & $500 \mathrm{mg}$ \\
\hline 2. Woche & $500 \mathrm{mg}$ & 0 & $500 \mathrm{mg}$ \\
\hline 3. Woche & $500 \mathrm{mg}$ & 0 & $2 \times 500 \mathrm{mg}$ \\
\hline anschließend & \multicolumn{2}{l}{$\begin{array}{l}\text { übliche Zieldosis: 2 x } 850 \mathrm{mg}, \text { evtl. Dosisänderung nach Körpergewicht, } \\
\text { Laborbefund, Verträglichkeit und Rücksprache mit dem behandelnden Arzt }\end{array}$}
\end{tabular}

Evtl. Gewichtsadaptation: $<60 \mathrm{~kg} \rightarrow 2 \times 500 \mathrm{mg},>60$ bis $100 \mathrm{~kg}$ oder BMI $<30 \mathrm{~kg} / \mathrm{m}^{2} \rightarrow 2 \times 850$ $\mathrm{mg},>100 \mathrm{~kg}$ oder $\mathrm{BMI}>30 \mathrm{~kg} / \mathrm{m}^{2} \rightarrow 2 \times 1.000 \mathrm{mg}$.

Zur besseren Verträglichkeit sollte Metformin zu oder nach der Mahlzeit eingenommen werden!

\section{Laborkontrollen}

- Vier Wochen nach Beginn der Metformin-Therapie

- Eine Woche nach Blutentnahme telefonische Befundbesprechung mit dem behandelndem Arzt, dann

- vierteljährlich BB, Leber-, Nieren- und Stoffwechselparametern, sowie Geschlechtshormone.

\section{Therapiedauer}

- Abhängig von Verträglichkeit und Erfolg. 


\subsection{Ferriman-Gallwey-Score}

\section{Einteilung nach Ferriman und Gallwey}

(betroffene Areale bitte ankreuzen)

1. Oberlippe: 1) wenig Haare außen 2) kleiner Bart außen 3) Oberlippenbart fast bis Mittellinie 4) Bart bis zur Mittellinie

2. Kinn : 1) vereinzelt Haare 2) Haaransammlung 3)+4) komplette Haardecke

3. Brust: vgl. rechte Graphik

4. Rücken: 1) einzelne Haare 2) mehrere Haare 3)+4) komplette Haardecke dicht

5. Lenden: 1) sakrales Haarpolster 2) Polster mit lateraler Ausdehnung 3) $3 / 4$ bedeckt 4) komplette Haardecke

6. Oberbauch: 1) wenig Haare an der Mittellinie 2) mehr als 1) aber noch im Bereich der Mittellinie 3)+4) halbe und komplette Haardecke

7. Unterbauch: 1) einige Haare an der Mittellinie 2) Strich von Haaren an der Mittellinie 3) Band von Haaren 4) umgekehrtes $V$

8. Oberarm: 1) diskrete Behaarung 2) mehr, noch keine geschlossene Haardecke 3)+4) komplette Haardecke

9. Oberschenkel : Einteilung wie bei 8 .

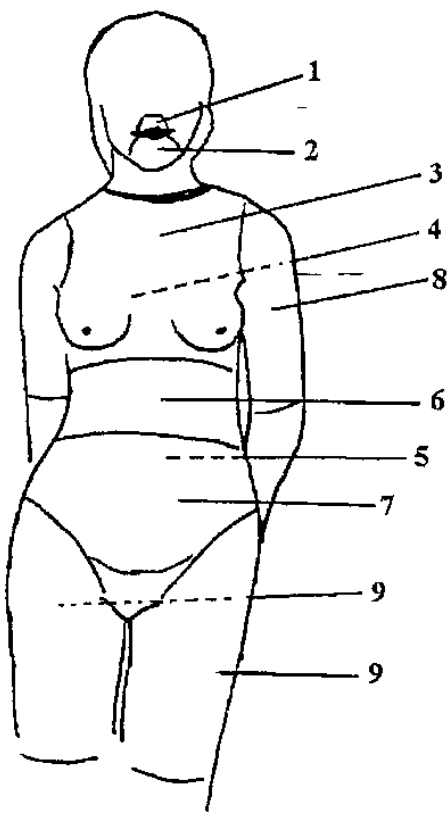

1)
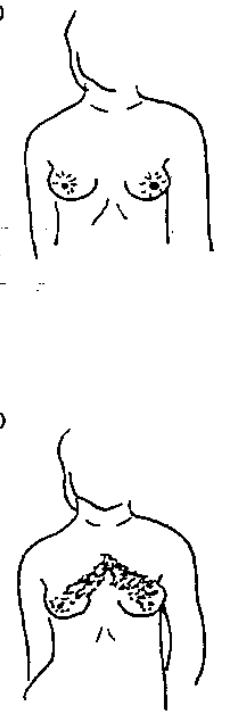

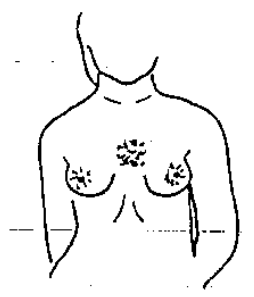

4)

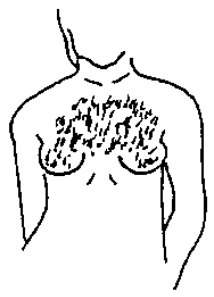




\section{Literaturverzeichnis}

Achard J, Thiers CE (1921): Le virilisme pilaire et son association à l'insuffisance glycolytique (diabète des femmes à barbe). Bull Acad Natl Med $\underline{86}, 51-56$

Acien P, Quereda F, Matallin P, Villarroya E, Lopez-Fernandez JA, Acien M, Mauri M, Alfayate R (1999): Insulin, androgens, and obesity in women with and without polycystic ovary syndrome: a heterogeneous group of disorders. Fertil Steril $\underline{72}, 32-$ 40

Alvarez-Blasco F, Botella-Carretero JI, San Millan JL, Escobar-Morreale HF (2006): Prevalence and characteristics of the polycystic ovary syndrome in overweight and obese women. Arch Intern Med 166, 2081-2086

Arslanian SA, Lewy V, Danadian K, Saad R (2002): Metformin therapy in obese adolescents with polycystic ovary syndrome and impaired Glukose tolerance: amelioration of exaggerated adrenal response to adrenocorticotropin with reduction of insulinemia/insulin resistance. J Clin Endocrinol Metab 87, 1555-1559

Azziz R, Woods KS, Reyna R, Key TJ, Knochenhauer ES, Yildiz BO (2004): The prevalence and features of the polycystic ovary syndrome in an unselected population. $\mathrm{J}$ Clin Endocrinol Metab 89, 2745-2749

Azziz R, Carmina E, Dewailly D, Diamanti-Kandarakis E, Escobar-Morreale HF, Futterweit W, Janssen OE, Legro RS, Norman RJ, Taylor AE, et al. (2006): Positions statement: criteria for defining polycystic ovary syndrome as a predominantly hyperandrogenic syndrome: an Androgen Excess Society guideline. J Clin Endocrinol Metab 91, 42374245

Azziz R, Carmina E, Dewailly D, Diamanti-Kandarakis E, Escobar-Morreale HF, Futterweit W, Janssen OE, Legro RS, Norman RJ, Taylor AE, et al. (2009): The Androgen Excess and PCOS Society criteria for the polycystic ovary syndrome: the complete task force report. Fertil Steril $\underline{91}$, 456-488

Badawy A, Elnashar A (2011): Treatment options for polycystic ovary syndrome. Int J Womens Health $\underline{3}, 25-35$

Badawy A, Abdel Aal I, Abulatta M (2009): Clomiphene citrate or letrozole for ovulation induction in women with polycystic ovarian syndrome: a prospective randomized trial. Fertil Steril $\underline{92}, 849-852$

Balen AH, Laven JS, Tan SL, Dewailly D (2003): Ultrasound assessment of the polycystic ovary: international consensus definitions. Hum Reprod Update $\underline{9}, 505-514$

Balkau B (2000): The DECODE study. Diabetes epidemiology: collaborative analysis of diagnostic criteria in Europe. Diabetes Metab 트, 282-286

Barry JA, Kuczmierczyk AR, Hardiman PJ (2011): Anxiety and depression in polycystic ovary syndrome: a systematic review and meta-analysis. Hum Reprod $\underline{26}, 2442-2451$

Benson S, Janssen OE, Hahn S, Tan S, Dietz T, Mann K, Pleger K, Schedlowski M, Arck $P C$, Elsenbruch $S$ (2008): Obesity, depression, and chronic low-grade inflammation in women with polycystic ovary syndrome. Brain Behav Immun 22, 177-184 
Blank SK, McCartney CR, Marshall JC (2006): The origins and sequelae of abnormal neuroendocrine function in polycystic ovary syndrome. Hum Reprod Update $\underline{12}, 351$ 361

Boomsma CM, Eijkemans MJ, Hughes EG, Visser GH, Fauser BC, Macklon NS (2006): A meta-analysis of pregnancy outcomes in women with polycystic ovary syndrome. Hum Reprod Update 12, 673-683

Bridger T, MacDonald S, Baltzer F, Rodd C (2006): Randomized placebo-controlled trial of metformin for adolescents with polycystic ovary syndrome. Arch Pediatr Adolesc Med $\underline{160}, 241-246$

Carmina E (2004): Diagnosis of polycystic ovary syndrome: from NIH criteria to ESHREASRM guidelines. Minerva Ginecol $\underline{56}, 1-6$

Carmina E, Lobo RA (2004): Use of fasting blood to assess the prevalence of insulin

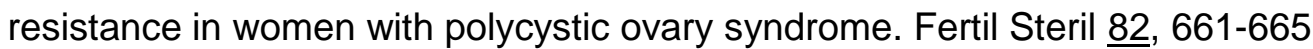

Carmina E, Legro RS, Stamets K, Lowell J, Lobo RA (2003): Difference in body weight between American and Italian women with polycystic ovary syndrome: influence of the diet. Hum Reprod 18, 2289-2293

Carmina E, Bucchieri S, Esposito A, Del Puente A, Mansueto P, Orio F, Di Fede G, Rini G (2007): Abdominal fat quantity and distribution in women with polycystic ovary syndrome and extent of its relation to insulin resistance. J Clin Endocrinol Metab $\underline{92}$, 2500-2505

Cascella T, Palomba S, De Sio I, Manguso F, Giallauria F, De Simone B, Tafuri D, Lombardi $G$, Colao A, Orio $F(2008)$ : Visceral fat is associated with cardiovascular risk in women with polycystic ovary syndrome. Hum Reprod 23, 153-159

Chen C, Smothers J, Lange A, Nestler JE, Strauss lii JF, Wickham lii EP (2010): Sex hormone-binding globulin genetic variation: associations with type 2 diabetes mellitus and polycystic ovary syndrome. Minerva Endocrinol $\underline{35}$, 271-280

Chhabra S, McCartney CR, Yoo RY, Eagleson CA, Chang RJ, Marshall JC (2005): Progesterone inhibition of the hypothalamic gonadotropin-releasing hormone pulse generator: evidence for varied effects in hyperandrogenemic adolescent girls. J Clin Endocrinol Metab 90, 2810-2815

Chittenden BG, Fullerton G, Maheshwari A, Bhattacharya S (2009): Polycystic ovary syndrome and the risk of gynaecological cancer: a systematic review. Reprod Biomed Online $\underline{19}$, 398-405

Christensen JT, Boldsen J, Westergaard JG (1997): Ovarian volume in gynecologically healthy women using no contraception, or using IUD or oral contraception. Acta Obstet Gynecol Scand 76, 784-789

Clark AM, Thornley B, Tomlinson L, Galletley C, Norman RJ (1998): Weight loss in obese infertile women results in improvement in reproductive outcome for all forms of fertility treatment. Hum Reprod 13, 1502-1505

Colilla S, Cox NJ, Ehrmann DA (2001): Heritability of insulin secretion and insulin action in women with polycystic ovary syndrome and their first degree relatives. J Clin Endocrinol Metab 86, 2027-2031

Coviello AD, Sam S, Legro RS, Dunaif A (2009): High prevalence of metabolic syndrome in first-degree male relatives of women with polycystic ovary syndrome is related to high rates of obesity. J Clin Endocrinol Metab 94, 4361-4366 
Dalla Man C, Campioni M, Polonsky KS, Basu R, Rizza RA, Toffolo G, Cobelli C (2005): Two-hour seven-sample oral Glukose tolerance test and meal protocol: minimal model assessment of beta-cell responsivity and insulin sensitivity in nondiabetic individuals. Diabetes $\underline{54}, 3265-3273$

Daniilidis A, Dinas K (2009): Long term health consequences of polycystic ovarian syndrome: a review analysis. Hippokratia $\underline{13}, 90-92$

DeUgarte CM, Bartolucci AA, Azziz R (2005): Prevalence of insulin resistance in the polycystic ovary syndrome using the homeostasis model assessment. Fertil Steril $\underline{83}$, 1454-1460

Diamanti-Kandarakis E, Christakou CD, Kandaraki E, Economou FN (2010): Metformin: an old medication of new fashion: evolving new molecular mechanisms and clinical implications in polycystic ovary syndrome. Eur J Endocrinol 162, 193-212

Douglas CC, Gower BA, Darnell BE, Ovalle F, Oster RA, Azziz R (2006): Role of diet in the treatment of polycystic ovary syndrome. Fertil Steril $\underline{85}, 679-688$

Dunaif A (2008): Drug insight: insulin-sensitizing drugs in the treatment of polycystic ovary syndrome-a reappraisal. Nat Clin Pract Endocrinol Metab 4, 272-283

Dunaif A, Segal KR, Futterweit W, Dobrjansky A (1989): Profound peripheral insulin resistance, independent of obesity, in polycystic ovary syndrome. Diabetes $\underline{38}, 1165-$ 1174

Eagleson CA, Gingrich MB, Pastor CL, Arora TK, Burt CM, Evans WS, Marshall JC (2000): Polycystic ovarian syndrome: evidence that flutamide restores sensitivity of the gonadotropin-releasing hormone pulse generator to inhibition by estradiol and progesterone. J Clin Endocrinol Metab 85, 4047-4052

Ehrmann DA, Kasza K, Azziz R, Legro RS, Ghazzi MN (2005): Effects of race and family history of type 2 diabetes on metabolic status of women with polycystic ovary syndrome. J Clin Endocrinol Metab 90, 66-71

Ehrmann DA, Liljenquist DR, Kasza K, Azziz R, Legro RS, Ghazzi MN (2006): Prevalence and predictors of the metabolic syndrome in women with polycystic ovary syndrome. $\mathrm{J}$ Clin Endocrinol Metab 91, 48-53

Escobar-Morreale HF, Luque-Ramirez M, Gonzalez F (2011): Circulating inflammatory markers in polycystic ovary syndrome: a systematic review and metaanalysis. Fertil Steril $\underline{95}, 1048-1058$ e1-2

Ferriman D, Gallwey JD (1961): Clinical assessment of body hair growth in women. J Clin Endocrinol Metab 21, 1440-1447

Fulghesu AM, Angioni S, Portoghese E, Milano F, Batetta B, Paoletti AM, Melis GB (2006): Failure of the homeostatic model assessment calculation score for detecting metabolic deterioration in young patients with polycystic ovary syndrome. Fertil Steril 86, 398-404

Ganie MA, Marwaha RK, Aggarwal R, Singh S (2010): High prevalence of polycystic ovary syndrome characteristics in girls with euthyroid chronic lymphocytic thyroiditis: a case-control study. Eur J Endocrinol 162, 1117-1122

Gannagé-Yared MH, Chedid R, Abs L (2011): Relation between androgens and cardiovascular risk factors in a young population. Clin Endocrinol (Oxf) $\underline{74}, 720-725$ 
Goodarzi MO, Dumesic DA, Chazenbalk G, Azziz R (2011): Polycystic ovary syndrome: etiology, pathogenesis and diagnosis. Nat Rev Endocrinol $\underline{7}, 219-231$

Hahn S, Quadbeck B, Elsenbruch S, Gärtner R, Finke R, Mann K, Janssen OE (2004): Metformin - ein effektiver Therapieansatz in der Behandlung des Polyzystischen Ovarsyndroms. Dtsch Med Wochenschr 129, 1059-1064

Hahn S, Tan S, Elsenbruch S, Quadbeck B, Herrmann BL, Mann K, Janssen OE (2005): Clinical and biochemical characterization of women with polycystic ovary syndrome in North Rhine-Westphalia. Horm Metab Res $\underline{37}$, 438-444

Halperin IJ, Kumar SS, Stroup DF, Laredo SE (2011): The association between the combined oral contraceptive pill and insulin resistance, dysglycemia and dyslipidemia in women with polycystic ovary syndrome: a systematic review and meta-analysis of observational studies. Hum Reprod 26, 191-201

Hermanns-Le T, Scheen A, Pierard GE (2004): Acanthosis nigricans associated with insulin resistance : pathophysiology and management. Am J Clin Dermatol $\underline{5}, 199-203$

Howren MB, Lamkin DM, Suls J (2009): Associations of depression with C-reactive protein, IL-1, and IL-6: a meta-analysis. Psychosom Med 71, 171-186

Huang A, Brennan K, Azziz R (2010): Prevalence of hyperandrogenemia in the polycystic ovary syndrome diagnosed by the National Institutes of Health 1990 criteria. Fertil Steril $\underline{93}, 1938-1941$

Hudecova M, Holte J, Moby L, Olovsson M, Stridsberg M, Larsson A, Berglund L, Berne C, Sundstrom Poromaa I (2011): Androgen levels, insulin sensitivity, and early insulin response in women with polycystic ovary syndrome: a long-term follow-up study. Fertil Steril $\underline{\text { 95, }}$ 1146-1148

Ibanez L, Lopez-Bermejo A, Diaz M, Marcos MV, de Zegher F (2011): Early metformin therapy (age 8-12 years) in girls with precocious pubarche to reduce hirsutism, androgen excess, and oligomenorrhea in adolescence. J Clin Endocrinol Metab $\underline{96}$, E1262-7

Janssen OE, Mehlmauer N, Hahn S, Offner AH, Gartner R (2004): High prevalence of autoimmune thyroiditis in patients with polycystic ovary syndrome. Eur $\mathrm{J}$ Endocrinol $150,363-369$

Jonard S, Robert Y, Cortet-Rudelli C, Pigny P, Decanter C, Dewailly D (2003): Ultrasound examination of polycystic ovaries: is it worth counting the follicles? Hum Reprod 18 , 598-603

Jovanovic VP, Carmina E, Lobo RA (2009): Not all women diagnosed with PCOS share the same cardiovascular risk profiles. Fertil Steril $\underline{94}$, 826-832

Kachuei M, Jafari F, Kachuei A, Keshteli AH (2011): Prevalence of autoimmune thyroiditis in patients with polycystic ovary syndrome. Arch Gynecol Obstet, im Druck

Kahsar-Miller MD, Nixon C, Boots LR, Go RC, Azziz R (2001): Prevalence of polycystic ovary syndrome (PCOS) in first-degree relatives of patients with PCOS. Fertil Steril $75,53-58$

Kamath MS, George K (2011): Letrozole or clomiphene citrate as first line for anovulatory infertility: a debate. Reprod Biol Endocrinol $\underline{9}, 86$ 
Katz A, Nambi SS, Mather K, Baron AD, Follmann DA, Sullivan G, Quon MJ (2000): Quantitative insulin sensitivity check index: a simple, accurate method for assessing insulin sensitivity in humans. J Clin Endocrinol Metab 85, 2402-2410

Key TJ, Pike MC, Moore JW, Bulbrook RD, Clark GM, Allen DS, Wang DY (1989): The relationships of SHBG with current and previous use of oral contraceptives and oestrogen replacement therapy. Contraception $\underline{39}, 179-186$

Legro RS, Driscoll D, Strauss JF, 3rd, Fox J, Dunaif A (1998a): Evidence for a genetic basis for hyperandrogenemia in polycystic ovary syndrome. Proc Natl Acad Sci U S A $\underline{95}$, 14956-14960

Legro RS, Finegood D, Dunaif A (1998b): A fasting Glukose to insulin ratio is a useful measure of insulin sensitivity in women with polycystic ovary syndrome. J Clin Endocrinol Metab 83, 2694-2698

Legro RS, Kunselman AR, Demers L, Wang SC, Bentley-Lewis R, Dunaif A (2002): Elevated dehydroepiandrosterone sulfate levels as the reproductive phenotype in the brothers of women with polycystic ovary syndrome. J Clin Endocrinol Metab 구, 2134-2138

Li HW, Brereton RE, Anderson RA, Wallace AM, Ho CK (2011): Vitamin D deficiency is common and associated with metabolic risk factors in patients with polycystic ovary syndrome. Metabolism $\underline{60}$, 1475-1481

Lowenstein EJ (2006): Diagnosis and management of the dermatologic manifestations of the polycystic ovary syndrome. Dermatol Ther $\underline{19}, 210-223$

Ludwig E (1977): Classification of the types of androgenetic alopecia (common baldness) occurring in the female sex. Br J Dermatol $97,247-254$

Luque-Ramirez M, Alpanes M, Escobar-Morreale HF (2010): The determinants of insulin sensitivity, beta-cell function, and Glukose tolerance are different in patients with polycystic ovary syndrome than in women who do not have hyperandrogenism. Fertil Steril $\underline{94}, 2214-2221$

Matthews DR, Hosker JP, Rudenski AS, Naylor BA, Treacher DF, Turner RC (1985): Homeostasis model assessment: insulin resistance and beta-cell function from fasting plasma Glukose and insulin concentrations in man. Diabetologia 28, 412-419

Morales AJ, Laughlin GA, Butzow T, Maheshwari H, Baumann G, Yen SS (1996): Insulin, somatotropic, and luteinizing hormone axes in lean and obese women with polycystic ovary syndrome: common and distinct features. J Clin Endocrinol Metab 81, 28542864

Moran LJ, Noakes M, Clifton PM, Tomlinson L, Galletly C, Norman RJ (2003): Dietary composition in restoring reproductive and metabolic physiology in overweight women with polycystic ovary syndrome. J Clin Endocrinol Metab $\underline{88}, 812-819$

Moran LJ, Misso ML, Wild RA, Norman RJ (2010): Impaired Glukose tolerance, type 2 diabetes and metabolic syndrome in polycystic ovary syndrome: a systematic review and meta-analysis. Hum Reprod Update 16, 347-363

Moran LJ, Pasquali R, Teede HJ, Hoeger KM, Norman RJ (2009): Treatment of obesity in polycystic ovary syndrome: a position statement of the Androgen Excess and Polycystic Ovary Syndrome Society. Fertil Steril $\underline{92}$, 1966-1982

O'Byrne S, Feely J (1990a): Effects of drugs on Glukose tolerance in non-insulin-dependent diabetics (Part I). Drugs 40, 6-18 
O'Byrne S, Feely J (1990b): Effects of drugs on Glukose tolerance in non-insulin-dependent diabetics (Part II). Drugs $\underline{40}, 203-219$

Oppelt PG, Mueller A, Jentsch K, Kronawitter D, Reissmann C, Dittrich R, Beckmann MW, Cupisti $S$ (2010): The effect of metformin treatment for 2 years without caloric restriction on endocrine and metabolic parameters in women with polycystic ovary syndrome. Exp Clin Endocrinol Diabetes 118, 633-637

Otta CF, Wior M, Iraci GS, Kaplan R, Torres D, Gaido MI, Wyse EP (2009): Clinical, metabolic, and endocrine parameters in response to metformin and lifestyle intervention in women with polycystic ovary syndrome: a randomized, double-blind, and placebo control trial. Gynecol Endocrinol 26, 173-178

Ovalle F, Azziz R (2002): Insulin resistance, polycystic ovary syndrome, and type 2 diabetes mellitus. Fertil Steril $\underline{77}, 1095-1105$

Págan YL, Srouji SS, Jimenez Y, Emerson A, Gill S, Hall JE (2006): Inverse relationship between luteinizing hormone and body mass index in polycystic ovarian syndrome: investigation of hypothalamic and pituitary contributions. J Clin Endocrinol Metab $\underline{91}$, $1309-1316$

Panzer C, Wise S, Fantini G, Kang D, Munarriz R, Guay A, Goldstein I (2006): Impact of oral contraceptives on sex hormone-binding globulin and androgen levels: a retrospective study in women with sexual dysfunction. J Sex Med $\underline{3}, 104-113$

Prapas N, Karkanaki A, Prapas I, Kalogiannidis I, Katsikis I, Panidis D (2009): Genetics of polycystic ovary syndrome. Hippokratia $\underline{13}, 216-223$

Pugeat M, Crave JC, Tourniaire J, Forest MG (1996): Clinical utility of sex hormone-binding globulin measurement. Horm Res $\underline{45}, 148-155$

Rizzo M, Berneis K, Spinas G, Rini GB, Carmina E (2009): Long-term consequences of polycystic ovary syndrome on cardiovascular risk. Fertil Steril $\underline{91}$, 1563-1567

Salley KE, Wickham EP, Cheang KI, Essah PA, Karjane NW, Nestler JE (2007): Glukose intolerance in polycystic ovary syndrome--a position statement of the Androgen Excess Society. J Clin Endocrinol Metab 92, 4546-4556

Schuring AN, Schulte N, Sonntag B, Kiesel L (2008): Androgens and insulin--two key players in polycystic ovary syndrome. Recent concepts in the pathophysiology and genetics of polycystic ovary syndrome. Gynakol Geburtshilfliche Rundsch $\underline{48}$, 9-15

Soriguer F, Rubio-Martin E, Fernandez D, Valdes S, Garcia-Escobar E, Martin-Nunez GM, Esteva I, Almaraz MC, Rojo-Martinez G (2012): Testosterone, SHBG and risk of type 2 diabetes in the second evaluation of the Pizarra cohort study. Eur $\mathrm{J}$ Clin Invest $\underline{42}$, 79-85

Srouji SS, Págan YL, D'Amato F, Dabela A, Jimenez Y, Supko JG, Hall JE (2007): Pharmacokinetic factors contribute to the inverse relationship between luteinizing hormone and body mass index in polycystic ovarian syndrome. J Clin Endocrinol Metab $\underline{\text { 92, }}$ 1347-1352

Stein I, Leventhal M (1935): Amenorrhea associated with bilateral polycystic ovaries. Am J Obstet Gynecol 29, 181-191

Tan S, Hahn S, Benson S, Dietz T, Lahner H, Moeller LC, Schmidt M, Elsenbruch S, Kimmig R, Mann K, et al. (2007): Metformin improves polycystic ovary syndrome symptoms irrespective of pre-treatment insulin resistance. Eur J Endocrinol 157, 669-676 
Tan S, Hahn S, Janssen OE (2008): Das polyzystische Ovarsyndrom - Metabolische Konsequenzen. Gynakol Geburtshilfliche Rundsch 4ㅇ, 16-23

Tan S, Scherag A, Janssen OE, Hahn S, Lahner H, Dietz T, Scherag S, Grallert H, Vogel Cl, Kimmig R, et al. (2010): Large effects on body mass index and insulin resistance of fat mass and obesity associated gene (FTO) variants in patients with polycystic ovary syndrome (PCOS). BMC Med Genet 11, 12

Tang T, Glanville J, Hayden CJ, White D, Barth JH, Balen AH (2006): Combined lifestyle modification and metformin in obese patients with polycystic ovary syndrome. A randomized, placebo-controlled, double-blind multicentre study. Hum Reprod 21, 8089

Tang T, Lord JM, Norman RJ, Yasmin E, Balen AH (2010): Insulin-sensitising drugs (metformin, rosiglitazone, pioglitazone, D-chiro-inositol) for women with polycystic ovary syndrome, oligo amenorrhoea and subfertility. Cochrane Database Syst Rev, CD003053

Taylor AE, McCourt B, Martin KA, Anderson EJ, Adams JM, Schoenfeld D, Hall JE (1997): Determinants of abnormal gonadotropin secretion in clinically defined women with polycystic ovary syndrome. J Clin Endocrinol Metab 82, 2248-2256

The Rotterdam ESHRE/ASRM-sponsored PCOS consensus workshop group (2004a): Revised 2003 consensus on diagnostic criteria and long-term health risks related to polycystic ovary syndrome. Fertil Steril $\underline{81}, 19-25$

The Rotterdam ESHRE/ASRM-sponsored PCOS consensus workshop group (2004b): Revised 2003 consensus on diagnostic criteria and long-term health risks related to polycystic ovary syndrome (PCOS). Hum Reprod $\underline{19}$, 41-47

Tian L, Shen H, Lu Q, Norman RJ, Wang J (2007): Insulin resistance increases the risk of spontaneous abortion after assisted reproduction technology treatment. J Clin Endocrinol Metab 92, 1430-1433

Tosi F, Dorizzi R, Castello R, Maffeis C, Spiazzi G, Zoppini G, Muggeo M, Moghetti P (2009): Body fat and insulin resistance independently predict increased serum C-reactive protein in hyperandrogenic women with polycystic ovary syndrome. Eur J Endocrinol $\underline{161}, 737-745$

Traub ML (2011): Assessing and treating insulin resistance in women with polycystic ovarian syndrome. World J Diabetes $\underline{2}$, 33-40

Veltman-Verhulst SM, van Haeften TW, Eijkemans MJ, de Valk HW, Fauser BC, Goverde AJ (2010): Sex hormone-binding globulin concentrations before conception as a predictor for gestational diabetes in women with polycystic ovary syndrome. Hum Reprod 25, 3123-3128

Waldstreicher J, Santoro NF, Hall JE, Filicori M, Crowley WF, Jr. (1988): Hyperfunction of the hypothalamic-pituitary axis in women with polycystic ovarian disease: indirect evidence for partial gonadotroph desensitization. J Clin Endocrinol Metab $\underline{66}$, 165172

Wallace TM, Levy JC, Matthews DR (2004): Use and abuse of HOMA modeling. Diabetes Care 27, 1487-1495

Wehr E, Pilz S, Schweighofer N, Giuliani A, Kopera D, Pieber TR, Obermayer-Pietsch B (2009): Association of hypovitaminosis D with metabolic disturbances in polycystic ovary syndrome. Eur J Endocrinol 161, 575-582 
Wehr E, Pieber TR, Obermayer-Pietsch B (2011a): Effect of vitamin D3 treatment on Glukose metabolism and menstrual frequency in polycystic ovary syndrome women: A pilot study. J Endocrinol Invest $\underline{34}, 757-763$

Wehr E, Trummer O, Giuliani A, Gruber HJ, Pieber TR, Obermayer-Pietsch B (2011b): Vitamin D-associated polymorphisms are related to insulin resistance and vitamin $\mathrm{D}$ deficiency in polycystic ovary syndrome. Eur J Endocrinol 164, 741-749

Weiss JM, Hadžiomerović D, Kreienberg R (2008): Syndrom der polyzystischen Ovarien (PCOS). Der Gynäkologe 41, 179-188

WHO (2000): Obesity: preventing and managing the global epidemic. Report of a WHO consultation. World Health Organ Tech Rep Ser 894, i-xii, 1-253

Yildiz BO, Gedik O (2001): Insulin resistance in polycystic ovary syndrome: hyperandrogenemia versus normoandrogenemia. Eur J Obstet Gynecol Reprod Biol $\underline{100}, 62-66$

Yildiz BO, Bolour S, Woods K, Moore A, Azziz R (2010): Visually scoring hirsutism. Hum Reprod Update 16, 51-64

Yildiz BO, Knochenhauer ES, Azziz R (2008): Impact of obesity on the risk for polycystic ovary syndrome. J Clin Endocrinol Metab 93, 162-168

Zawadski JK, Dunaif A: Diagnostic criteria for polycystic ovary syndrome: towards a rational approach; in: Polycystic Ovary Syndrome; hrsg. v. Dunaif A, Givens JR, Haseltine FP, Merriam GE; Blackwell Scientific, Boston 1992, 377-384 


\section{Danksagung}

An dieser Stelle möchte ich mich bei allen bedanken, die zum Gelingen dieser Arbeit beigetragen haben.

In erster Linie möchte ich mich bei Frau Prof. Dr. med. Heide Siggelkow für die freundliche Überlassung des Promotionsthemas und für die stets sehr gute Betreuung und Unterstützung bedanken. Insbesondere die konstruktiven Gespräche im Endokrinologikum sowie die zeitnahen Korrekturen mit den für mich hilfreichen Kommentaren haben zum erfolgreichen Abschluss dieser Arbeit beigetragen.

Zudem bedanke ich mich besonders bei Herrn Dipl.-Wi.-Math. Hans-Joachim Helms und den wissenschaftlichen Hilfskräften der Abteilung für Medizinische Statistik der UMG: Erstens für die wiederholten Beratungen zur statistischen Auswertung, sowie zweitens für die Geduld, mir das effektive Arbeiten mit Statistica vertraut zu machen. Weiterhin gilt mein Dank den Mitarbeiterinnen des Endokrinologikums Göttingen für die jederzeit unkomplizierte Hilfe beim Umgang mit der Praxissoftware.

Herrn Prof. Dr. Dr. med. Bernd Hinney danke ich für die freundliche Überlassung bzw. Modifizierung seiner Abbildungen zum Thema PCOS.

Und schließlich möchte ich mich noch besonders bei allen Korrekurlesern, insbesondere bei meiner Familie und bei meiner Freundin Sabine, bedanken. 